\title{
CLASH: WEAK-LENSING SHEAR-AND-MAGNIFICATION ANALYSIS OF 20 GALAXY CLUSTERS*
}

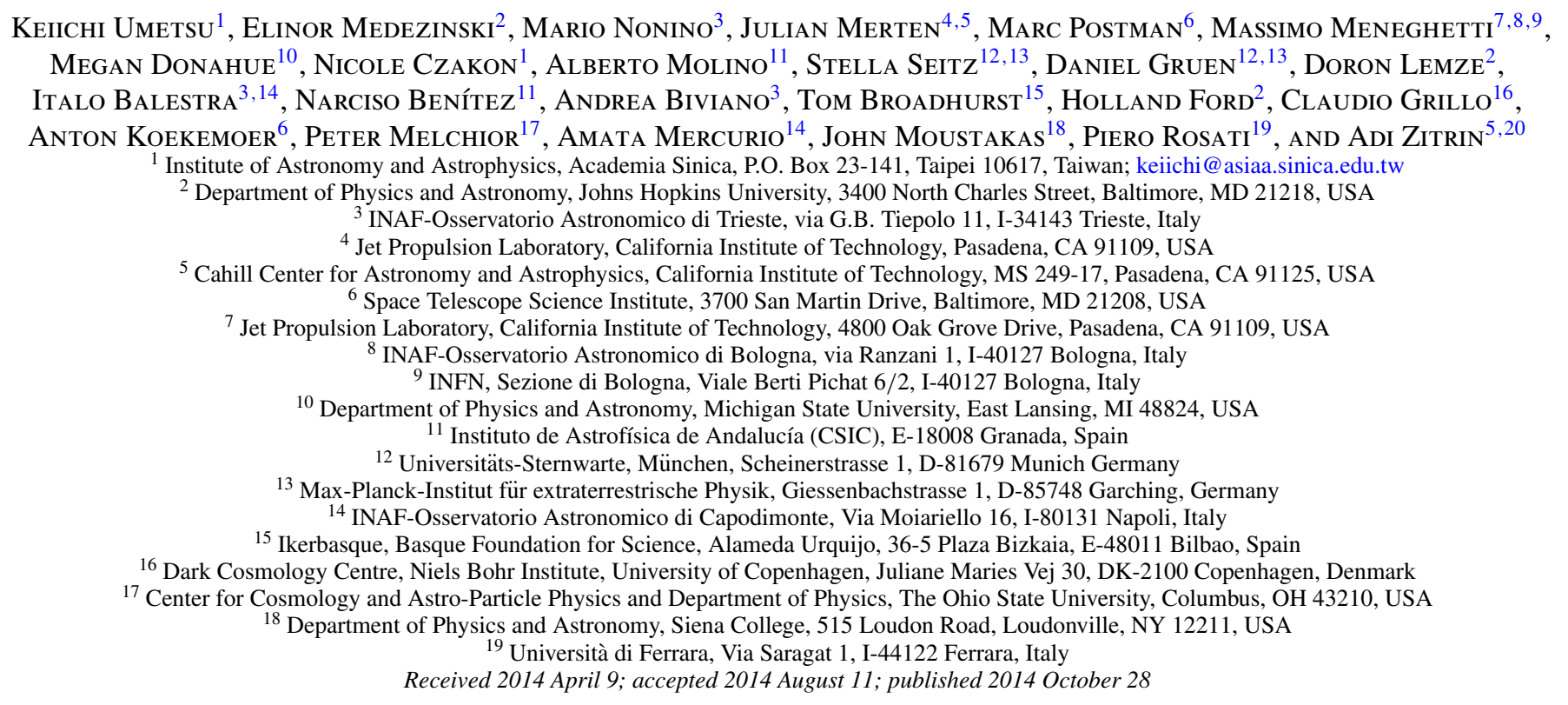

\section{ABSTRACT}

We present a joint shear-and-magnification weak-lensing analysis of a sample of $16 \mathrm{X}$-ray-regular and 4 highmagnification galaxy clusters at $0.19 \lesssim z \lesssim 0.69$ selected from the Cluster Lensing And Supernova survey with Hubble (CLASH). Our analysis uses wide-field multi-color imaging, taken primarily with Suprime-Cam on the Subaru Telescope. From a stacked-shear-only analysis of the X-ray-selected subsample, we detect the ensembleaveraged lensing signal with a total signal-to-noise ratio of $\simeq 25$ in the radial range of $200-3500 \mathrm{kpc} h^{-1}$, providing integrated constraints on the halo profile shape and concentration-mass relation. The stacked tangential-shear signal is well described by a family of standard density profiles predicted for dark-matter-dominated halos in gravitational equilibrium, namely, the Navarro-Frenk-White (NFW), truncated variants of NFW, and Einasto models. For the NFW model, we measure a mean concentration of $c_{200 c}=4.01_{-0.32}^{+0.35}$ at an effective halo mass of $M_{200 c}=1.34_{-0.09}^{+0.10} \times 10^{15} M_{\odot}$. We show that this is in excellent agreement with $\Lambda$ cold dark matter $(\Lambda \mathrm{CDM})$ predictions when the CLASH X-ray selection function and projection effects are taken into account. The best-fit Einasto shape parameter is $\alpha_{\mathrm{E}}=0.191_{-0.068}^{+0.071}$, which is consistent with the NFW-equivalent Einasto parameter of $\sim 0.18$. We reconstruct projected mass density profiles of all CLASH clusters from a joint likelihood analysis of shear-and-magnification data and measure cluster masses at several characteristic radii assuming an NFW density profile. We also derive an ensemble-averaged total projected mass profile of the X-ray-selected subsample by stacking their individual mass profiles. The stacked total mass profile, constrained by the shear+magnification data, is shown to be consistent with our shear-based halo-model predictions, including the effects of surrounding large-scale structure as a two-halo term, establishing further consistency in the context of the $\Lambda$ CDM model.

Key words: cosmology: observations - dark matter - galaxies: clusters: general - gravitational lensing: weak

Online-only material: color figures

\section{INTRODUCTION}

Clusters of galaxies represent the largest cosmic structures that have reached a state in the vicinity of gravitational equilibrium. The abundance of massive clusters as a function of redshift is highly sensitive to the amplitude and growth rate of primordial density fluctuations, as well as the cosmic volume-redshift relation (Haiman et al. 2001). Clusters therefore play a fundamental role in examining cosmological models, allowing several independent tests of any viable cosmology, including the current

\footnotetext{
* Based in part on data collected at the Subaru Telescope, which is operated by the National Astronomical Society of Japan.

${ }^{20}$ Hubble Fellow.
}

concordance $\Lambda$ cold dark matter $(\Lambda \mathrm{CDM})$ model defined in the framework of general relativity.

Clusters, by virtue of their enormous mass, serve as giant physics laboratories for astronomers to explore the role and nature of dark matter (DM), the physics governing the final state of self-gravitating collisionless systems in an expanding universe (Gunn \& Gott 1972; Navarro et al. 1996; Taylor \& Navarro 2001; Lapi \& Cavaliere 2009; Hjorth \& Williams 2010), and screening mechanisms in long-range modified models of gravity whereby general relativity is restored (Narikawa \& Yamamoto 2012).

A key ingredient of such cosmological tests is the mass distribution of clusters. In the standard picture of hierarchical 
structure formation, cluster halos are located at dense nodes where the filaments intersect and are still forming through successive mergers of smaller halos and through smooth accretion of matter along their surrounding large-scale structure (LSS).

The standard $\Lambda C D M$ model and its variants provide observationally testable predictions for the structure of DM-dominated halos. Cosmological $N$-body simulations of collisionless DM have established a nearly self-similar form for the spherically averaged density profile $\rho(r)$ of equilibrium halos (Navarro et al. 1996, hereafter NFW) over a wide range of halo masses, with some intrinsic variance associated with mass accretion histories of individual halos (Jing \& Suto 2000; Merritt et al. 2006; Graham et al. 2006; Navarro et al. 2010; Gao et al. 2012; Ludlow et al. 2013). The degree of mass concentration, $c_{200 \mathrm{c}}=r_{200 \mathrm{c}} / r_{\mathrm{s}},{ }^{21}$ is predicted to correlate with halo mass since DM halos that are more massive collapse later on average, when the mean background density of the universe is correspondingly lower (Bullock et al. 2001; Neto et al. 2007). Accordingly, cluster-sized halos are predicted to be less concentrated than less massive systems and to have typical concentrations of $c_{200 \mathrm{c}} \simeq 3-4$, compared to $c_{200 \mathrm{c}} \simeq 5$ for group-sized halos (Duffy et al. 2008; Bhattacharya et al. 2013).

Unlike individual galaxies, massive clusters are not expected to be significantly affected by baryonic gas cooling (Blumenthal et al. 1986; Mead et al. 2010; Duffy et al. 2010; Lau et al. 2011; Blanchard et al. 2013) because the majority ( 80\%) of baryons in clusters constitute a hot, X-ray-emitting phase of the intracluster medium, in which the high temperature and low density prevent efficient cooling and gas contraction. Consequently, for clusters in a state of quasi-equilibrium, the form of their total mass profiles reflects closely the underlying DM distribution.

Clusters act as gravitational lenses, producing various detectable lensing effects, including deflection, shearing, and magnifying of the images of distant background sources. There is a weak-lensing regime where lensing effects can be linearly related to the gravitational potential so that it is possible to determine mass distributions in a model-free way. Weak lensing shear offers a direct means of mapping the mass distribution of clusters (Umetsu et al. 1999; Bartelmann \& Schneider 2001; Hoekstra et al. 2013) irrespective of the physical nature, composition, and state of lensing matter, providing a direct probe for testing well-defined predictions of halo structure.

Lensing magnification provides complementary, independent observational alternatives to gravitational shear (Broadhurst et al. 1995; Umetsu \& Broadhurst 2008; Van Waerbeke et al. 2010; Umetsu et al. 2011a; Hildebrandt et al. 2011; Ford et al. 2012; Zemcov et al. 2013; Coupon et al. 2013). Gravitational magnification influences the surface density of background sources, expanding the area of sky, and enhancing the observed flux of background sources (Broadhurst et al. 1995). The former effect reduces the effective observing area in the source plane, decreasing the source counts per solid angle. The latter effect increases the number of sources above the limiting flux because the limiting luminosity $L_{\lim }(z)$ at any background redshift $z$ lies effectively at a fainter limit, $L_{\lim }(z) / \mu(z)$, with $\mu(z)$ the magnification factor. The net effect is known as "magnification bias" and depends on the steepness of the luminosity function.

In practice, magnification bias can be used in combination with weak-lensing shear to obtain a model-free determination

\footnotetext{
21 The quantity $r_{200 c}$ is defined as the radius within which the mean density is 200 times the critical density $\rho_{c}(z)$ of the universe at the cluster redshift $z$, and $r_{\mathrm{s}}$ is a scale radius at which $d \ln \rho / d \ln r=-2$.
}

of the projected mass profiles of clusters (Schneider et al. 2000; Umetsu \& Broadhurst 2008; Umetsu et al. 2011a; Umetsu 2013), effectively breaking degeneracies inherent in a standard weaklensing analysis based on shape information alone (Section 2.1; see also Schneider \& Seitz 1995). Our earlier work has established that deep multi-color imaging allows us to simultaneously detect the observationally independent shear and magnification signals efficiently from the same data. The combination of shear and magnification allows us not only to perform consistency checks of observational systematics but also to enhance the precision and accuracy of cluster mass estimates (Rozo \& Schmidt 2010; Umetsu et al. 2012; Umetsu 2013).

The Cluster Lensing And Supernova survey with Hubble (CLASH; Postman et al. 2012) has been designed to map the DM distribution in a representative sample of 25 clusters, by using high-quality strong- and weak-lensing data, in combination with wide-field imaging from Suprime-Cam on the Subaru Telescope (e.g., Umetsu et al. 2011a, 2011b, 2012). CLASH is a 524-orbit multi-cycle treasury Hubble Space Telescope (HST) program to observe 25 clusters at $0.18<z<0.89$, each in 16 filters with the Wide Field Camera 3 (Kimble et al. 2008) and the Advanced Camera for Surveys (ACS; Ford et al. 2003).

The CLASH sample is drawn largely from the Abell and MACS cluster catalogs (Abell 1958; Abell et al. 1989; Ebeling et al. 2001, 2007, 2010). Twenty CLASH clusters were X-ray selected to be massive and to have a regular X-ray morphology. This selection is suggested to minimize the strong bias toward high concentrations in previously well-studied clusters selected for their strong-lensing strength, allowing us to meaningfully examine the $c-M$ relation for a cluster sample that is largely free of lensing bias (Postman et al. 2012). A further sample of five clusters were selected by their high lens magnification properties, with the primary goal of detecting and studying highredshift background galaxies magnified by the cluster potential.

In this paper, we present a joint shear-and-magnification weak-lensing analysis of a sample of 16 X-ray-regular and 4 high-magnification clusters targeted in the CLASH survey. Our analysis uses wide-field multi-band imaging obtained primarily with Subaru/Suprime-Cam. In particular, we aim at using the combination of shear and magnification information to study ensemble-averaged mass density profiles of CLASH clusters and compare with theoretical expectations in the context of the $\Lambda C D M$ cosmology. This work has two companion papers: the strong-lensing and weak-shear study of CLASH clusters by Merten et al. (2014) and the detailed characterization of numerical simulations of CLASH clusters by Meneghetti et al. (2014, hereafter M14).

The paper is organized as follows. In Section 2, we summarize the basic theory of cluster weak gravitational lensing. In Section 3, we present the formalism we use for our weak-lensing analysis, which combines shear and magnification information. In Section 4, we describe the observational data set, its reduction, weak-lensing shape measurements, and the selection of background galaxies. In Section 5, we describe our joint shear-andmagnification analysis of 20 CLASH clusters. In Section 6, we carry out stacked weak-lensing analyses of our X-ray-selected subsample to study their ensemble-averaged mass distribution. Section 7 is devoted to the discussion of the results. Finally, a summary is given in Section 8.

Throughout this paper, we use the $\mathrm{AB}$ magnitude system and adopt a concordance $\Lambda$ CDM cosmology with $\Omega_{\mathrm{m}}=0.27$, $\Omega_{\Lambda}=0.73$, and $h \equiv 0.7 h_{70}=0.7$ (Komatsu et al. 2011), where $H_{0}=100 \mathrm{~h} \mathrm{~km} \mathrm{~s}^{-1} \mathrm{Mpc}^{-1}$. We use the standard notation 
$M_{\Delta_{\mathrm{c}}}\left(M_{\Delta_{\mathrm{m}}}\right)$ to denote the total mass enclosed within a sphere of radius $r_{\Delta_{\mathrm{c}}}\left(r_{\Delta_{\mathrm{m}}}\right)$, within which the mean density is $\Delta_{\mathrm{c}}\left(\Delta_{\mathrm{m}}\right)$ times the critical (mean background) density of the universe at the cluster redshift. All quoted errors are $68.3 \%(1 \sigma)$ confidence limits (CL) unless otherwise stated.

\section{WEAK-LENSING BASICS}

\subsection{Convergence, Shear, and Magnification}

The image deformation due to weak lensing is characterized by the convergence $\kappa$, which describes the isotropic focusing of light rays, and the gravitational shear $\gamma(\boldsymbol{\theta})=|\gamma| e^{2 i \phi}$ with spin-2 rotational symmetry (e.g., Bartelmann \& Schneider 2001). The lensing convergence is $\kappa(\boldsymbol{\theta})=\Sigma(\boldsymbol{\theta}) / \Sigma_{\mathrm{c}}$, the projected mass density $\Sigma(\boldsymbol{\theta})$ in units of the critical surface mass density for lensing,

$$
\Sigma_{\mathrm{c}}=\frac{c^{2}}{4 \pi G} \frac{D_{\mathrm{s}}}{D_{\mathrm{l}} D_{\mathrm{ls}}} \equiv \frac{c^{2}}{4 \pi G D_{\mathrm{l}}} \beta^{-1},
$$

with $D_{\mathrm{l}}, D_{\mathrm{s}}$, and $D_{\mathrm{ls}}$ the proper angular diameter distances from the observer to the lens, the observer to the source, and the lens to the source, respectively. The distance ratio $\beta=D_{\mathrm{ls}} / D_{\mathrm{s}}$ represents the geometric strength of cluster lensing for a source at redshift $z ; \beta(z)=0$ for unlensed objects, $z \leqslant z_{1}$.

The shear $\gamma(\boldsymbol{\theta})$ induces a quadrupole anisotropy of the background images, which can be observed from ellipticities of background galaxies. Given an arbitrary circular loop of radius $\theta$, the tangential shear $\gamma_{+}$and the $45^{\circ}$ rotated component $\gamma_{\times}$averaged around the loop satisfy the following identity (Kaiser 1995):

$$
\gamma_{+}(\theta)=\kappa(<\theta)-\kappa(\theta) \equiv \Delta \Sigma_{+}(\theta) / \Sigma_{\mathrm{c}}, \quad \gamma_{\times}(\theta)=0,
$$

with $\kappa(\theta)=\Sigma(\theta) / \Sigma_{\mathrm{c}}$ the azimuthally averaged convergence at radius $\theta, \kappa(<\theta)=\Sigma(<\theta) / \Sigma_{\mathrm{c}}$ the average convergence interior to $\theta$, and $\Delta \Sigma_{+}(\theta)=\Sigma_{\mathrm{c}} \gamma_{+}(\theta)$ the differential surface mass density. In general, the observable quantity for quadrupole weak lensing is not $\gamma$ but the reduced gravitational shear,

$$
g(\boldsymbol{\theta})=\frac{\gamma(\boldsymbol{\theta})}{1-\kappa(\boldsymbol{\theta})},
$$

which is invariant under $\kappa(\boldsymbol{\theta}) \rightarrow \lambda \kappa(\boldsymbol{\theta})+1-\lambda$ and $\gamma(\boldsymbol{\theta}) \rightarrow$ $\lambda \gamma(\boldsymbol{\theta})$ with an arbitrary constant $\lambda \neq 0$, known as the mass-sheet degeneracy (see Schneider \& Seitz 1995). This degeneracy can be broken, for example, ${ }^{22}$ by measuring the magnification

$$
\mu(\boldsymbol{\theta})=\frac{1}{[1-\kappa(\boldsymbol{\theta})]^{2}-|\gamma(\boldsymbol{\theta})|^{2}},
$$

which transforms as $\mu(\boldsymbol{\theta}) \rightarrow \lambda^{2} \mu(\boldsymbol{\theta})$.

\subsection{Source Redshift Distribution}

For statistical weak-lensing measurements, we consider populations of source galaxies with respective redshift distribution functions $\bar{N}(z)$. The mean lensing depth for a given population is given by

$$
\langle\beta\rangle=\left[\int_{0}^{\infty} d z w(z) \bar{N}(z) \beta(z)\right]\left[\int_{0}^{\infty} d z w(z) \bar{N}(z)\right]^{-1},
$$

\footnotetext{
22 Alternatively, the constant $\lambda$ can be determined such that the $\kappa$ averaged over the outermost cluster region vanishes, if a sufficiently wide sky coverage is available. Or, one may constrain $\lambda$ such that the enclosed mass within a certain aperture is consistent with cluster mass estimates from some other observations (e.g., Umetsu \& Futamase 2000).
}

where $w(z)$ is a weight factor. In general, we apply different size, magnitude, and color cuts in source selection for measuring the shear and magnification effects, resulting in different $\bar{N}(z)$. In contrast to the former effect, the latter does not require source galaxies to be spatially resolved, but it does require a stringent flux limit against incompleteness effects.

We introduce the relative lensing strength of a source population with respect to a fiducial source in the far background as (Bartelmann \& Schneider 2001)

$$
\langle W\rangle=\langle\beta\rangle / \beta_{\infty},
$$

with $\beta_{\infty} \equiv \beta\left(z \rightarrow \infty ; z_{1}\right)$. The associated critical surface mass density is $\Sigma_{\mathrm{c}, \infty}\left(z_{1}\right)=c^{2} /\left(4 \pi G D_{1} \beta_{\infty}\right)$. The source-averaged convergence and shear fields are then expressed as $\langle\kappa(\theta)\rangle=$ $\langle W\rangle \kappa_{\infty}(\boldsymbol{\theta})$ and $\langle\gamma(\boldsymbol{\theta})\rangle=\langle W\rangle \gamma_{\infty}(\boldsymbol{\theta})$, using those in the farbackground limit. Hereafter, we use the far-background lensing fields, $\kappa_{\infty}$ and $\gamma_{\infty}$, to describe the projected mass distribution of clusters.

\section{CLUSTER ANALYSIS METHODOLOGY}

In this section we present the formalism that we use for our weak-lensing analysis, which combines complementary shear and magnification information. In Sections 3.1 and 3.2, we first describe our methods for measuring cluster lensing profiles as a function of cluster radius. In Section 3.3, we outline our Bayesian approach for reconstructing the projected mass profile from a joint likelihood analysis of shear+magnification measurements. In Section 3.4, we describe our stacked analysis formalism and procedures for determining the ensemble-averaged lensing profiles.

\subsection{Tangential-distortion Profile}

We construct azimuthally averaged radial profiles of the tangential distortion $g_{+}$and the $45^{\circ}$ rotated component $g_{\times}$as functions of cluster radius $\theta$ (Umetsu \& Broadhurst 2008; Umetsu 2013). In the absence of higher-order effects, weak lensing induces only curl-free tangential distortions (Section 2.1). In practice, the presence of $\times$ modes can be used to check for systematic errors.

The tangential distortion averaged over the source redshift distribution $\bar{N}_{g}(z)$ is expressed as $\left\langle g_{+}\right\rangle=\left[\int_{0}^{\infty} d z g_{+}(W[z]) \bar{N}_{g}(z)\right]$ $\left[\int_{0}^{\infty} d z \bar{N}_{g}(z)\right]^{-1}$. The averaging operator with respect to $\bar{N}(z)$ acts nonlinearly on the redshift-dependent components in the cluster lensing observables. In the mildly nonlinear regime, it is often sufficient to apply a low-order approximation using low-order moments of the source-averaged lensing depth.

Specifically, we use the following approximation for the nonlinear corrections to the source-averaged reduced shear profile (Seitz \& Schneider 1997; Umetsu 2013):

$$
\left\langle g_{+}\right\rangle \approx \frac{\langle W\rangle_{g}\left[\kappa_{\infty}(<\theta)-\kappa_{\infty}(\theta)\right]}{1-\kappa_{\infty}(\theta)\left\langle W^{2}\right\rangle_{g} /\langle W\rangle_{g}}=\frac{\left\langle\gamma_{+}\right\rangle}{1-f_{W, g}\langle\kappa\rangle},
$$

where $\langle W\rangle_{g}$ is the relative lensing strength (see Section 2.2) averaged over the population $N_{g}(z)$ of source galaxies, $f_{W, g} \equiv$ $\left\langle W^{2}\right\rangle_{g} /\langle W\rangle_{g}^{2}$ is a dimensionless quantity of the order unity, $\langle\kappa\rangle=\langle W\rangle_{g} \kappa_{\infty}$, and $\left\langle\gamma_{+}\right\rangle=\langle W\rangle_{g} \gamma_{+, \infty}$.

\subsection{Magnification-bias Profile}

\subsubsection{Magnification Bias}

Since a given flux limit corresponds to different intrinsic luminosities at different source redshifts, count measurements of 
distinctly different background populations probe different regimes of magnification-bias effects. Deep multi-band photometry allows us to explore the faint end of the intrinsic luminosity function of red galaxies at $z \sim 1$ (e.g., Figures 11-13 of Ilbert et al. 2010). For a flux-limited sample of the faint red background population, the effect of magnification bias is dominated by the geometric area distortion because relatively few fainter galaxies can be magnified into the sample, thus resulting in a net count depletion (Taylor et al. 1998; Broadhurst et al. 2005; Umetsu \& Broadhurst 2008; Umetsu et al. 2011a, 2012; Coe et al. 2012; Medezinski et al. 2013).

In the present work, we perform count-depletion measurements using flux-limited samples of red background galaxies. If the magnitude shift $\delta m=2.5 \log _{10} \mu$ induced by magnification is small compared to that on which the logarithmic slope of the source luminosity function varies, the source number counts can be locally approximated by a power law at the limiting magnitude $m$. The magnification bias at redshift $z$ is then given by (Broadhurst et al. 1995)

$$
N_{\mu}(\boldsymbol{\theta}, z ;<m)=\bar{N}_{\mu} \mu^{2.5 s-1}(\boldsymbol{\theta}, z) \equiv \bar{N}_{\mu} b_{\mu}(\boldsymbol{\theta}, z ; s),
$$

where $\bar{N}_{\mu}=\bar{N}_{\mu}(z ;<m)$ is the unlensed mean source counts and $s$ is the logarithmic count slope evaluated at $m, s=$ $d \log _{10} \bar{N}_{\mu}(z ;<m) / d m$. In the regime where $2.5 s \ll 1$, the effect of magnification bias is dominated by the geometric expansion of the sky area and hence is not sensitive to the exact form of the intrinsic source luminosity function.

Taking into account the spread of $\bar{N}_{\mu}(z)$, we express the population-averaged magnification bias as $\left\langle b_{\mu}\right\rangle=$ $\left[\int_{0}^{\infty} d z b_{\mu}(W[z]) \bar{N}_{\mu}(z)\right]\left[\int_{0}^{\infty} d z \bar{N}_{\mu}(z)\right]^{-1}$. In this work, we use the following equation to interpret the observed count-depletion measurements (Umetsu 2013):

$$
\left\langle b_{\mu}(\boldsymbol{\theta})\right\rangle=N_{\mu}(\boldsymbol{\theta} ;<m) / \bar{N}_{\mu}(<m) \approx\left\langle\mu^{-1}(\boldsymbol{\theta})\right\rangle^{1-2.5 s_{\mathrm{eff}}},
$$

with $s_{\text {eff }}=d \log _{10} \bar{N}_{\mu}(<m) / d m$ the effective count slope defined in analogy to Equation (8). Equation (9) is exact for $s_{\text {eff }}=0$ and gives a good approximation for depleted background populations with $s_{\text {eff }} \ll 0.4$ (Umetsu 2013). Furthermore, the source-averaged inverse magnification is approximated as (Umetsu 2013)

$$
\begin{aligned}
\left\langle\mu^{-1}\right\rangle & =(1-\langle\kappa\rangle)^{2}-|\langle\gamma\rangle|^{2}+\left(f_{W, \mu}-1\right)\left(\langle\kappa\rangle^{2}-|\langle\gamma\rangle|^{2}\right) \\
& \approx(1-\langle\kappa\rangle)^{2}-|\langle\gamma\rangle|^{2}
\end{aligned}
$$

where $f_{W, \mu} \equiv\left\langle W^{2}\right\rangle_{\mu} /\langle W\rangle_{\mu}^{2}$ is of the order unity, $\langle\kappa\rangle=$ $\langle W\rangle_{\mu} \kappa_{\infty}$, and $\langle\gamma\rangle=\langle W\rangle_{\mu} \gamma_{\infty}$. The error associated with the approximation above is $\left\langle\Delta \mu^{-1}\right\rangle=\left(f_{W, \mu}-1\right)\left(\langle\kappa\rangle^{2}-|\langle\gamma\rangle|^{2}\right) \equiv$ $\Delta f_{W, \mu}\left(\langle\kappa\rangle^{2}-|\langle\gamma\rangle|^{2}\right)$, which is much smaller than unity for source populations of our concern $\left(\Delta f_{W, \mu} \sim O\left(10^{-2}\right)\right)$ in the regime where $\langle\kappa\rangle \sim|\langle\gamma\rangle| \sim O\left(10^{-1}\right)$.

\subsubsection{Number-count Depletion}

In practical observations, the nonvanishing and unresolved angular correlation on small angular scales can lead to a significant increase in the variance of counts in cells (van Waerbeke 2000), which can be much larger than the lensing signal in a given cell. To obtain a clean measure of the lensing signal, such intrinsic variance needs to be downweighted (Umetsu \& Broadhurst 2008) and averaged over a sufficiently large sky area.
This local clustering noise can be largely overcome by performing an azimuthal average around the cluster (Umetsu et al. 2011a; Umetsu 2013). Here we calculate the surface number density of background sources $n_{\mu}(\theta)=d N_{\mu}(\theta) / d \Omega$ as a function of cluster radius, by azimuthally averaging the source counts in cells, $N_{\mu}(\boldsymbol{\theta} ;<m)$. The source-averaged magnification bias is then expressed as $\left\langle n_{\mu}(\theta)\right\rangle=\bar{n}_{\mu}\left\langle\mu^{-1}(\theta)\right\rangle^{1-2.5 s_{\mathrm{eff}}}$ with $\bar{n}_{\mu}=d \bar{N}_{\mu}(<m) / d \Omega$ the unlensed mean surface number density of background sources.

In this work, we adopt the following prescription.

1. A positive tail of $>v \sigma$ cells is excluded in each annulus by iterative $\sigma$ clipping with $v=2.5$ to reduce the effect of intrinsic angular clustering of source galaxies. We take the systematic change between the mean counts estimated with and without $\nu \sigma$ clipping as a systematic error, $\sigma_{\mu}^{\text {sys }}(\theta)=\left|n_{\mu}^{(\nu)}(\theta)-n_{\mu}^{(\infty)}(\theta)\right| / \nu$, where $n_{\mu}^{(\nu)}(\theta)$ and $n_{\mu}^{(\infty)}(\theta)$ are the clipped and unclipped mean counts in the annulus $\theta$, respectively. The statistical Poisson uncertainty $\sigma_{\mu}^{\text {stat }}(\theta)$ is estimated from the clipped mean counts.

2. An additional contribution to the uncertainty from the intrinsic clustering of source galaxies, $\sigma_{\mu}^{\text {int }}(\theta)$, is estimated empirically from the variance in each annulus due to variations of the counts $N_{\mu}(\boldsymbol{\theta})$ along the azimuthal direction.

3 . Each grid cell is weighted by the fraction of its area lying within the respective annular bins. We use Monte Carlo integration to calculate the area fractions for individual cells.

4. Masking of background galaxies due to cluster galaxies, foreground objects, and saturated pixels is properly taken into account and corrected for, by calculating at each annulus $f_{\text {mask }}(\theta)=\Delta \Omega_{\text {mask }}(\theta) / \Delta \Omega_{\text {tot }}(\theta)$, with $\Delta \Omega_{\text {mask }}(\theta)$ the area of masked regions in the annulus and $\Delta \Omega_{\mathrm{tot}}(\theta)$ the total area of the annulus. In our analysis, we use Method B of Appendix A developed by Umetsu et al. (2011a).

The errors are combined in quadrature as $\sigma_{\mu}^{2}=\left(\sigma_{\mu}^{\text {stat }}\right)^{2}+$ $\left(\sigma_{\mu}^{\text {int }}\right)^{2}+\left(\sigma_{\mu}^{\text {sys }}\right)^{2}$. We note that the $\sigma_{\mu}^{\text {sys }}$ contribution may account for (1) strong contamination by background clusters projected near the line of sight and (2) spurious excess counts of red galaxies due perhaps to spatial variation of the photometric zero point and/or to residual flat-field errors.

Finally, we apply the correction to the number counts for the masking effects by $n_{\mu}(\theta) \rightarrow n_{\mu}(\theta) /\left[1-f_{\text {mask }}(\theta)\right]$ and $\sigma_{\mu}(\theta) \rightarrow$ $\sigma_{\mu}(\theta) /\left[1-f_{\text {mask }}(\theta)\right]$. Similarly, this correction is applied to the mean background counts $\bar{n}_{\mu}$ and its total uncertainty. The typical level of this correction is about $8 \%$ in our weak-lensing observations (see Section 5.2).

\subsection{Bayesian Mass-profile Reconstruction}

\subsubsection{Joint Likelihood Function}

In the Bayesian framework of Umetsu et al. (2011a), the signal is described by a vector $s$ of parameters containing the binned convergence profile $\left\{\kappa_{\infty, i}\right\}_{i=1}^{N}$ given by $N$ binned $\kappa$ values and the average convergence enclosed by the innermost aperture radius $\theta_{\min }, \kappa_{\infty, \min } \equiv \kappa_{\infty}\left(<\theta_{\min }\right)$, so that

$$
\boldsymbol{s}=\left\{\kappa_{\infty, \min }, \kappa_{\infty, i}\right\}_{i=1}^{N} \equiv \Sigma_{\mathrm{c}, \infty}^{-1} \boldsymbol{\Sigma},
$$

specified by $(N+1)$ parameters.

The joint likelihood function $\mathcal{L}(s)$ for multi-probe lensing observations is given as a product of their separate likelihoods, 
$\mathcal{L}=\mathcal{L}_{g} \mathcal{L}_{\mu}$, with $\mathcal{L}_{g}$ and $\mathcal{L}_{\mu}$ the likelihood functions for $\left\{\left\langle g_{+, i}\right\rangle\right\}_{i=1}^{N}$ and $\left\{\left\langle n_{\mu, i}\right\rangle\right\}_{i=1}^{N}$, defined as

$$
\begin{aligned}
& \ln \mathcal{L}_{g}(\boldsymbol{s})=-\frac{1}{2} \sum_{i=1}^{N} \frac{\left[\left\langle g_{+, i}\right\rangle-\hat{g}_{+, i}(\boldsymbol{s}, \boldsymbol{c})\right]^{2}}{\sigma_{+, i}^{2}}, \\
& \ln \mathcal{L}_{\mu}(\boldsymbol{s})=-\frac{1}{2} \sum_{i=1}^{N} \frac{\left[\left\langle n_{\mu, i}\right\rangle-\hat{n}_{\mu, i}(\boldsymbol{s}, \boldsymbol{c})\right]^{2}}{\sigma_{\mu, i}^{2}},
\end{aligned}
$$

with $\left(\hat{g}_{+}, \hat{n}_{\mu}\right)$ the theoretical predictions for the corresponding observations and $c$ the calibration nuisance parameters to marginalize over,

$$
\boldsymbol{c}=\left\{\langle W\rangle_{g}, f_{W, g},\langle W\rangle_{\mu}, \bar{n}_{\mu}, s_{\mathrm{eff}}\right\} .
$$

For each parameter of the model $s$, we consider a flat uninformative prior with a lower bound of $s=0$. Additionally, we account for the calibration uncertainty in the observational parameters $\boldsymbol{c}$.

\subsubsection{Estimators and Covariance Matrix}

We implement our method using a Markov Chain Monte Carlo (MCMC) approach with Metropolis-Hastings sampling, by following the prescription outlined in Umetsu et al. (2011a). The shear+magnification method has been tested (Umetsu 2013) against synthetic weak-lensing catalogs from simulations of analytical NFW lenses performed using the public package GLAFIC (Oguri 2010). The results suggest that, when the mass-sheet degeneracy is broken, both maximum-likelihood (ML) and Bayesian marginal maximum a posteriori probability (MMAP) solutions produce reliable reconstructions with unbiased profile measurements, so that this method is not sensitive to the choice and form of priors. In the presence of a systematic bias in the background-density constraint $\left(\bar{n}_{\mu}\right)$, the global ML estimator is less sensitive to systematic effects than MMAP and provides robust reconstructions (Section 7.4.2).

On the basis of our simulations, we thus use the global ML estimator for determination of the mass profile. In our error analysis we take into account statistical, systematic, and cosmic-noise contributions to the total covariance matrix $C_{i j} \equiv \operatorname{Cov}\left(s_{i}, s_{j}\right)$ as

$$
C=C^{\mathrm{stat}}+C^{\mathrm{sys}}+C^{\mathrm{lss}}
$$

where $C^{\text {stat }}$ is estimated from the posterior MCMC samples, $C^{\text {sys }}$ accounts for systematic errors due primarily to the mass-sheet uncertainty,

$$
C_{i j}^{\mathrm{sys}}=\left(\boldsymbol{s}_{\mathrm{ML}}-\boldsymbol{s}_{\mathrm{MMAP}}\right)_{i}^{2} \delta_{i j}
$$

with $s_{\text {ML }}$ and $s_{\text {MMAP }}$ the ML and MMAP solutions, respectively, and $C^{\mathrm{lss}}$ is the cosmic-noise covariance due to uncorrelated LSS projected along the line of sight (Hoekstra 2003; Hoekstra et al. 2011; Umetsu et al. 2011b). For a given depth of weak-lensing observations, the impact of cosmic noise is most important when the cluster signal itself is small (Hoekstra 2003), that is, when nearby clusters are studied, or when data at large cluster radii are examined.

The $C^{\mathrm{lss}}$ matrix is computed for a given source population as outlined in Umetsu et al. (2011b), ${ }^{23}$ using the nonlinear matter power spectrum of Smith et al. (2003) for the Wilkinson Microwave Anisotropy Probe (WMAP) seven-year cosmology (Komatsu et al. 2011).

\footnotetext{
23 Note that $C^{\mathrm{lss}}$ is calculated for our weak-lensing source populations and then scaled to the reference far-background source plane.
}

\subsection{Stacked-lensing Formalism}

\subsubsection{Stacked Tangential-shear Profile}

First, we derive an expression for the averaged $\Delta \Sigma_{+}$profile from stacking of tangential distortion signals around the cluster centers, following the general procedure of Umetsu et al. (2011b). For a given cluster sample, we center the shear catalogs on the respective cluster centers and construct their individual distortion profiles $\left\langle\boldsymbol{g}_{+}\right\rangle$in physical proper length units across the range $R=D_{1} \theta=\left[R_{\min }, R_{\max }\right]$. As we shall see below, our choice of stacking in physical length units is to reduce systematic errors in determining the ensemble-averaged cluster mass profile from stacked-lensing measurements (Okabe et al. 2013), although this choice is not optimized for the signal-tonoise ratio $(\mathrm{S} / \mathrm{N})$ of the stacked signal.

For each cluster, we express the covariance matrix $\left(C_{+}\right)_{i j}$ of $\left\langle\boldsymbol{g}_{+}\right\rangle$as a sum of the contributions from the shape noise $\left(C_{+}^{\text {stat }}\right)$ and the cosmic shear $\left(C_{+}^{\text {lss }}\right)$ due to uncorrelated LSS projected along the line of sight (Hoekstra 2003), $C_{+}=C_{+}^{\text {stat }}+C_{+}^{\text {lss }}$, where $\left(C_{+}^{\text {stat }}\right)_{i j}=\sigma_{+, i}^{2} \delta_{i j}$ is estimated from bootstrap resampling of the background source catalog and $C_{+}^{\text {lss }}$ is computed for a given source population (see Section 3.3.2). This cosmic noise is correlated between radial bins but can be overcome by stacking an ensemble of clusters along independent lines of sight (Umetsu et al. 2011b).

Since the noise in different clusters is uncorrelated, the tangential distortion profiles of individual clusters can be coadded according to (Umetsu et al. 2011b)

$$
\left\langle\widehat{\Delta \Sigma_{+}}\right\rangle=\left(\sum_{n} \mathcal{W}_{+, n}\right)^{-1}\left(\sum_{n} \mathcal{W}_{+, n} \widehat{\Delta \Sigma_{+, n}}\right),
$$

where $\langle\langle\ldots\rangle\rangle$ denotes the sensitivity-weighted average over all clusters in the sample, $\widehat{\Delta \boldsymbol{\Sigma}_{+, n}}=\Sigma_{\mathrm{c}, n}\left\langle\boldsymbol{g}_{+, n}\right\rangle$, and $\mathcal{W}_{+, n}$ is the shear-sensitivity matrix of the $n$th cluster

$$
\left(\mathcal{W}_{+, n}\right)_{i j} \equiv \Sigma_{\mathrm{c}, n}^{-2}\left(C_{+, n}^{-1}\right)_{i j}
$$

with $C_{+, n}$ the covariance matrix of the $n$th $\left\langle\boldsymbol{g}_{+}\right\rangle$profile.

The statistical covariance matrix $\mathcal{C}_{+}^{\text {stat }}$ for $\left\langle\left\langle\widehat{\Delta \Sigma}_{+}\right\rangle\right\rangle$is estimated from bootstrap resampling of the cluster sample in Equation (17), which accounts for the statistical total variation of the mean mass profile averaged over the sample. Additionally, we include in our error analysis the photo- $z$ uncertainties on the mean depth calibration, $\mathcal{C}^{\text {sys }}$, and the net cosmic-noise covariance,

$$
\mathcal{C}_{+}^{\mathrm{lss}}=\left[\sum_{n} \Sigma_{\mathrm{c}, n}^{-2}\left(C_{+, n}^{\mathrm{lss}}\right)^{-1}\right]^{-1}
$$

Finally, the full covariance matrix for $\left\langle\left\langle\widehat{\Delta \Sigma}_{+}\right\rangle\right\rangle$is obtained as

$$
\mathcal{C}_{+}=\mathcal{C}_{+}^{\text {stat }}+\mathcal{C}_{+}^{\text {sys }}+\mathcal{C}_{+}^{\text {lss }} \text {. }
$$

The relation between the observable lens distortion and the lensing fields is nonlinear (Equation (3)), so that the stacked $\left\langle\left\langle\widehat{\Delta \Sigma_{+}}\right\rangle\right\rangle$profile is nonlinearly related to the averaged lensing fields. Expanding the right-hand side of Equation (17) and taking the ensemble average, we obtain the next-to-leadingorder correction as

$$
\begin{aligned}
\left\langle\left\langle\widehat{\Delta \Sigma_{+}}\right\rangle\right\rangle & =\mathrm{E}\left[\Delta \Sigma_{+}\right]+\left\langle\left\langle\Sigma_{\mathrm{c}}^{-1}\right\rangle\right\rangle \mathrm{E}\left[\boldsymbol{\Sigma} \Delta \Sigma_{+}\right] \\
& \approx \mathrm{E}\left[\Delta \Sigma_{+}\right] /\left(1-\left\langle\left\langle\Sigma_{\mathrm{c}}^{-1}\right\rangle \mathrm{E}[\boldsymbol{\Sigma}]\right),\right.
\end{aligned}
$$


Table 1

Cluster Sample

\begin{tabular}{|c|c|c|c|c|c|c|c|}
\hline Cluster & $z_{1}$ & $\begin{array}{c}\text { R.A. }^{\mathrm{a}} \\
(\mathrm{J} 2000.0)\end{array}$ & $\begin{array}{c}\text { Decl. }^{\mathrm{a}} \\
(\mathrm{J} 2000.0)\end{array}$ & $\begin{array}{l}k_{\mathrm{B}} T^{\mathrm{b}} \\
(\mathrm{keV})\end{array}$ & $\begin{array}{c}\text { Offset }^{\mathrm{c}} \\
\left(\mathrm{kpc} h^{-1}\right)\end{array}$ & Filters $^{\mathrm{d}}$ & $\begin{array}{l}\text { WL Band } \\
\left(\text { Seeing }\left(^{\prime \prime}\right)\right)\end{array}$ \\
\hline \multicolumn{8}{|c|}{ X-ray selected } \\
\hline A383 & 0.187 & 02:48:03.40 & $-03: 31: 44.9$ & $6.5 \pm 0.24$ & 1.4 & $\underline{B}_{\mathrm{J}} V_{\mathrm{J}} \underline{R}_{\mathrm{C}} I_{\mathrm{C}} i^{\prime} \underline{z}^{\prime}$ & $i^{\prime}(0.57)$ \\
\hline A209 & 0.206 & $01: 31: 52.54$ & $-13: 36: 40.4$ & $7.3 \pm 0.54$ & 3.9 & $\underline{B}_{\mathrm{J}} V_{\mathrm{J}} \underline{R}_{\mathrm{C}} i^{\prime} \underline{z}^{\prime}$ & $R_{\mathrm{C}}(0.61)$ \\
\hline A2261 & 0.224 & $17: 22: 27.18$ & $+32: 07: 57.3$ & $7.6 \pm 0.30$ & 4.0 & $\underline{B}_{\mathrm{J}} \underline{V}_{\mathrm{J}} \underline{R}_{\mathrm{C}} i^{+} z^{+}$ & $R_{\mathrm{C}}(0.56)$ \\
\hline RX J2129.7+0005 & 0.234 & $21: 29: 39.96$ & $+00: 05: 21.2$ & $5.8 \pm 0.40$ & 6.3 & $\underline{B}_{\mathrm{J}} V_{\mathrm{J}} \underline{R}_{\mathrm{C}} i^{\prime} \underline{z}^{\prime}$ & $R_{\mathrm{C}}(0.53)$ \\
\hline A611 & 0.288 & 08:00:56.82 & $+36: 03: 23.6$ & $7.9 \pm 0.35$ & 1.6 & $\underline{B}_{\mathrm{J}} V_{\mathrm{J}} \underline{R}_{\mathrm{C}} I_{\mathrm{C}} \bar{i}^{\prime} \underline{z}^{\prime}$ & $R_{\mathrm{C}}(0.65)$ \\
\hline MS2137-2353 & 0.313 & $21: 40: 15.17$ & $-23: 39: 40.2$ & $5.9 \pm 0.30$ & 1.7 & $\underline{B}_{\mathrm{J}} V_{\mathrm{J}} \underline{R}_{\mathrm{C}} I_{\mathrm{C}} \underline{z}^{\prime}$ & $R_{\mathrm{C}}(0.60)$ \\
\hline RX J2248.7-4431 & 0.348 & $22: 48: 43.96$ & $-44: 31: 51.3$ & $12.4 \pm 0.60$ & 15.9 & $U_{877} \underline{B}_{842} V_{843} \underline{R}_{844} I_{879} z_{846} i^{*} \underline{z}^{*}$ & $R_{844}(0.81)$ \\
\hline MACS J1115.9+0129 & 0.352 & $11: 15: 51.90$ & $+01: 29: 55.1$ & $8.0 \pm 0.40$ & 9.5 & $\underline{B}_{\mathrm{J}} V_{\mathrm{J}} \underline{R}_{\mathrm{C}} I_{\mathrm{C}} \underline{z}^{\prime}$ & $R_{\mathrm{C}}(0.67)$ \\
\hline MACS J1931.8-2635 & 0.352 & $19: 31: 49.62$ & $-26: 34: 32.9$ & $6.7 \pm 0.40$ & 4.3 & $\underline{B}_{\mathrm{J}} V_{\mathrm{J}} \underline{R}_{\mathrm{C}} I_{\mathrm{C}} \underline{z}^{\prime}$ & $R_{\mathrm{C}}(0.72)$ \\
\hline RXJ1532.9+3021 & 0.363 & $15: 32: 53.78$ & $+30: 20: 59.4$ & $5.5 \pm 0.40$ & 2.5 & $\underline{B}_{\mathrm{J}} V_{\mathrm{J}} \underline{R}_{\mathrm{C}} I_{\mathrm{C}} \underline{z}^{\prime}$ & $R_{\mathrm{C}}(0.57)$ \\
\hline MACS J1720.3+3536 & 0.391 & $17: 20: 16.78$ & $+35: 36: 26.5$ & $6.6 \pm 0.40$ & 13.3 & $\underline{B}_{\mathrm{J}} V_{\mathrm{J}} \underline{R}_{\mathrm{C}} I_{\mathrm{C}} \underline{z}^{\prime}$ & $R_{\mathrm{C}}(0.79)$ \\
\hline MACS J0429.6-0253 & 0.399 & $04: 29: 36.05$ & $-02: 53: 06.1$ & $6.0 \pm 0.44$ & 13.6 & $\underline{V}_{\mathrm{J}} \underline{R}_{\mathrm{C}} \underline{I}_{\mathrm{C}}$ & $R_{\mathrm{C}}(0.75)$ \\
\hline MACS J1206.2-0847 & 0.440 & $12: 06: 12.15$ & $-08: 48: 03.4$ & $10.8 \pm 0.60$ & 8.7 & $\underline{B}_{\mathrm{J}} V_{\mathrm{J}} \underline{R}_{\mathrm{C}} I_{\mathrm{C}} \underline{z}^{\prime}$ & $I_{\mathrm{C}}(0.71)$ \\
\hline MACS J0329.7-0211 & 0.450 & $03: 29: 41.56$ & $-02: 11: 46.1$ & $8.0 \pm 0.50$ & 9.8 & $\underline{B}_{\mathrm{J}} V_{\mathrm{J}} \underline{R}_{\mathrm{C}} I_{\mathrm{C}} \underline{z}^{\prime}$ & $R_{\mathrm{C}}(0.47)$ \\
\hline RX J1347.5-1145 & 0.451 & $13: 47: 31.05$ & $-11: 45: 12.6$ & $15.5 \pm 0.60$ & 29.4 & $g^{\star} \underline{B}_{\mathrm{J}} V_{\mathrm{J}} \underline{R}_{\mathrm{C}} I_{\mathrm{C}} \underline{z}^{\prime}$ & $R_{\mathrm{C}}(0.71)$ \\
\hline MACS J0744.9+3927 & 0.686 & $07: 44: 52.82$ & $+39: 27: 26.9$ & $8.9 \pm 0.80$ & 12.6 & $\underline{B}_{\mathrm{J}} V_{\mathrm{J}} \underline{R}_{\mathrm{C}} I_{\mathrm{C}} i^{\prime} \underline{z}^{\prime}$ & $R_{\mathrm{C}}(0.71)$ \\
\hline \multicolumn{8}{|c|}{ High magnification } \\
\hline MACS J0416.1-2403 & 0.396 & $04: 16: 08.38$ & $-24: 04: 20.8$ & $7.5 \pm 0.80$ & 82.3 & $\underline{B}_{\mathrm{J}} \underline{R}_{\mathrm{C}} \underline{z}^{\prime}$ & $\overline{R_{\mathrm{C}}(0.55)}$ \\
\hline MACS J1149.5+2223 & 0.544 & $11: 49: 35.69$ & $+22: 23: 54.6$ & $8.7 \pm 0.90$ & 10.8 & $\underline{B}_{\mathrm{J}} V_{\mathrm{J}} \underline{R}_{\mathrm{C}} \underline{z}^{\prime} K_{\mathrm{S}}$ & $R_{\mathrm{C}}(0.80)$ \\
\hline MACS J0717.5+3745 & 0.548 & $07: 17: 32.63$ & $+37: 44: 59.7$ & $12.5 \pm 0.70$ & 100.2 & $u^{\star} \underline{B}_{\mathrm{J}} V_{\mathrm{J}} \underline{R}_{\mathrm{C}} \bar{i}^{\prime} \underline{z}^{\prime} J K_{\mathrm{S}}$ & $R_{\mathrm{C}}(0.79)$ \\
\hline MACS J0647.7+7015 & 0.584 & $06: 47: 50.27$ & $+70: 14: 55.0$ & $13.3 \pm 1.80$ & 25.4 & $\underline{B}_{\mathrm{J}} V_{\mathrm{J}} \underline{R}_{\mathrm{C}} I_{\mathrm{C}} i^{\prime} \underline{z}^{\prime}$ & $R_{\mathrm{C}}(0.64)$ \\
\hline
\end{tabular}

Notes.

a Optical cluster center.

b X-ray temperature from Postman et al. (2012).

c Projected offset between the X-ray and optical centers.

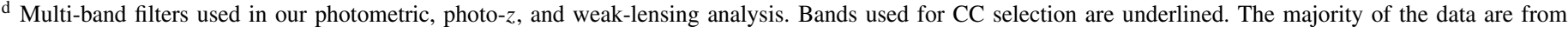
Subaru/Suprime-Cam. Bands from complementary facilities are described in Table 2.

e Band used for weak-lensing shape measurements and seeing FWHM in the full stack of images.

where $E[. .$.$] denotes the ensemble average, and we have used$ the trace approximation $\left(\mathcal{W}_{+, n}\right)_{i j} \propto \delta_{i j} \operatorname{tr}\left(\mathcal{W}_{+, n}\right)$ in the first line of Equation (21); $\left\langle\left\langle\Sigma_{\mathrm{c}}^{-1}\right\rangle\right\rangle$ is defined as

$$
\left\langle\left\langle\Sigma_{\mathrm{c}}^{-1}\right\rangle\right\rangle=\frac{\sum_{n} \operatorname{tr}\left(\mathcal{W}_{+, n}\right) \Sigma_{\mathrm{c}, n}^{-1}}{\sum_{n} \operatorname{tr}\left(\mathcal{W}_{+, n}\right)},
$$

where the statistical weight $\operatorname{tr}\left(\mathcal{W}_{+, n}\right)$ is proportional to $1 / \Sigma_{\mathrm{c}, n}^{2}$ (Equation (18)). We note that $\operatorname{tr}\left(\mathcal{W}_{+, n}\right)$ is independent of the cluster mass when stacking in physical length units (Okabe et al. 2010, 2013; Umetsu et al. 2011b; Oguri et al. 2012). On the other hand, as discussed by Okabe et al. (2013), stacking in length units scaled to $r_{\Delta}$ weights the contribution of each cluster to each radial bin in a nonlinear and model-dependent manner, such that $\operatorname{tr} \mathcal{W}_{+} \propto r_{\Delta}^{2} \propto M_{\Delta}^{2 / 3}$ when $C_{+}$is dominated by the shape noise contribution $\left(C_{+}^{\text {stat }}\right)$.

In this work, we shall use Equations (21) and (22) to obtain a best-fit model for a given $\left\langle\left\langle\widehat{\Delta \Sigma_{+}}\right\rangle\right\rangle$. The $\left\langle\left\langle\Delta \Sigma_{+}\right\rangle\right\rangle$is then obtained as

$$
\left.\left\langle\left\langle\Delta \Sigma_{+}\right\rangle\right\rangle \approx\left\langle\widehat{\Delta \Sigma_{+}}\right\rangle\right\rangle-\left\langle\left\langle\Sigma_{\mathrm{c}}^{-1}\right\rangle\right\rangle\left(\Sigma \Delta \Sigma_{+}\right),
$$

where the nonlinear correction $\left(\Sigma \Delta \Sigma_{+}\right)$is calculated using the best-fit solution to $\left\langle\left\langle\widehat{\Delta \Sigma_{+}}\right\rangle\right\rangle$in Equation (21).

\subsubsection{Stacked Mass Profile}

Having obtained the mass density profiles $\boldsymbol{\Sigma}$ of individual clusters from combined weak-lensing shear and magnification measurements (Section 3), we can stack the clusters to produce an averaged radial mass profile.
Following Umetsu et al. (2011b), we re-evaluate the mass profiles of individual clusters in proper length units across the range $R=\left[R_{\min }, R_{\max }\right]$ and construct $\Sigma=\left\{\Sigma\left(<R_{\min }\right), \Sigma\left(R_{i}\right)\right\}_{i=1}^{N}$ on the same radial grid for all clusters. Stacking an ensemble of clusters is expressed as

$$
\langle\langle\Sigma\rangle\rangle=\left(\sum_{n} \mathcal{W}_{n}\right)^{-1}\left(\sum_{n} \mathcal{W}_{n} \Sigma_{n}\right),
$$

where $\mathcal{W}_{n}$ is the $n$th sensitivity matrix defined as $\left(\mathcal{W}_{n}\right)_{i j} \equiv \Sigma_{(\mathrm{c}, \infty) n}^{-2}\left(C_{n}^{-1}\right)_{i j}$ with $C_{n}$ the total covariance matrix (Equation (15)) of the $n$th cluster. ${ }^{24}$

Finally, the full covariance matrix $\mathcal{C}$ for the stacked $\langle\langle\Sigma\rangle\rangle$ profile can be obtained in a similar manner as for $\left\langle\left\langle\Delta \Sigma_{+}\right\rangle\right\rangle$ (Equation (20)), accounting for the profile variations in individual clusters, observational uncertainties, photo- $z$ uncertainties on the mean-depth calibration, and the net cosmic-noise contribution.

\section{CLUSTER SAMPLE AND OBSERVATIONS}

\subsection{Cluster Sample}

Our cluster sample comprises two subsamples, one with 16 $\mathrm{X}$-ray-selected clusters and another with four high-magnification clusters, both taken from the CLASH sample (Postman et al. 2012). Table 1 provides a summary of the cluster properties in

\footnotetext{
24 Since the covariance matrix $C$ is defined for the far-background convergence $\kappa_{\infty}$, the associated critical surface mass density too is a far-background quantity, $\Sigma_{\mathrm{c}, \infty}=\Sigma_{\mathrm{c}}(z \rightarrow \infty)$.
} 
our sample. In this work, the optical cluster center is taken to be the location of the brightest cluster galaxy (BCG) when a single dominant central galaxy is found. Otherwise, it is defined as the center of the brightest red-sequence-selected cluster galaxies (MACS J0717.5+3745; Medezinski et al. 2013; MACS J0416.1-2403).

All clusters in the X-ray-selected subsample have X-ray temperatures greater than $5 \mathrm{keV}$ and show a smooth, regular morphology in their X-ray brightness distribution (Postman et al. 2012). Importantly, the X-ray selection allows us to reduce a bias toward higher concentrations as found in lensing-selected clusters, where selecting clusters according to their lensing properties can introduce an orientation bias in favor of prolate structure pointed to the observer, as this orientation boosts the projected mass density, and a selection bias toward intrinsically overconcentrated clusters (Broadhurst et al. 2008; Oguri et al. 2009; Meneghetti et al. 2010a). Our X-ray criteria also ensure well-defined cluster centers, reducing the effects of cluster miscentering, where smoothing from the miscentering effects flattens the recovered lensing profiles below the offset scale and thus leads to an underestimation of the derived concentration and inner-slope parameters.

\subsection{Wide-field Imaging Observations}

Our CLASH wide-field imaging data rely primarily on observations taken with the Suprime-Cam imager $\left(34^{\prime} \times 27^{\prime}\right.$; Miyazaki et al. 2002) at the prime focus of the $8.3 \mathrm{~m}$ Subaru Telescope. We combine both existing archival data taken from SMOKA $^{25}$ with observations acquired by the team on the nights of 2010 March 17-18 (S10A-019), 2010 November 4-6 (S10B059), and 2012 July 22 (S12A-063). A good fraction of the Subaru data were taken as part of the Weighing the Giants (WtG) project and were independently analyzed in their series of papers (von der Linden et al. 2014; Kelly et al. 2014; Applegate et al. 2014). The complete multi-band filter information for all the clusters is summarized in Table 1 . The filter naming conventions and description are given in Table 2.

To improve the accuracy of our photometric redshift (photo- $z$ ) measurements, we also retrieved, reduced, and used optical data taken with the Megaprime/MegaCam (RX J1347.5-1145, MACS J0717.5+3745) and near-IR data with the WIRCam (MACS J1149.5+2223, MACS J0717.5+3745) on the CanadaFrance-Hawaii Telescope (CFHT), where available from the CFHT archive. ${ }^{26}$ For A2261, additional bands were available from the KPNO telescope archive ${ }^{27}$ to augment the existing Subaru data (see Coe et al. 2012).

For our southernmost cluster, RXC J2248-4431, which is not observable from Subaru, we rely on data obtained by Gruen et al. (2013) with the Wide-Field Imager (WFI) at the ESO $2.2 \mathrm{~m} \mathrm{MPG} / \mathrm{ESO}$ telescope at La Silla. We use the same coadded mosaic images built by Gruen et al. (2013) but conduct an independent analysis adopting substantially different approaches in performing shape measurements, shear calibration, photometry, photo- $z$ measurements, background selection, and lensing reconstruction, which is based on the combination of shear and magnification effects. We also obtained data with the IMACS camera (Bigelow \& Dressler 2003) on the Magellan $6 \mathrm{~m}$ telescope.

\footnotetext{
25 http://smoka.nao.ac.jp

26 This research used the facilities of the Canadian Astronomy Data Centre operated by the National Research Council of Canada with the support of the Canadian Space Agency.

27 http://portal-nvo.noao.edu/home/main
}

Table 2

Multi-band Filter Description

\begin{tabular}{lcl}
\hline \hline Telescope/Instrument & Filter Name & Filter Description \\
\hline Subaru/Suprime-Cam & $B_{\mathrm{J}}$ & Johnson $B$-band \\
& $V_{\mathrm{J}}$ & Johnson $V$-band \\
& $R_{\mathrm{C}}$ & Cousins $R$-band \\
& $I_{\mathrm{C}}$ & Cousins $I$-band \\
& $i$ & SDSS $i$-band \\
& $z^{\prime}$ & SDSS $z$-band \\
\hline KPNO Mayall/MOSAIC-I & $i^{+}$ & SDSS $i$-band \\
& $z^{+}$ & SDSS $z$-band \\
\hline ESO/WFI & $U_{877}$ & $U / 50$-band \\
& $B_{842}$ & Johnson $B$-band \\
& $V_{843}$ & Johnson $V$-band \\
& $R_{844}$ & Cousins $R$-band \\
& $I_{879}$ & Cousins $I$-band \\
& $z_{846}$ & $z+/ 61$-band \\
\hline Magellan/IMACS & $i^{*}$ & SDSS $i$-band \\
& $z^{*}$ & SDSS $z$-band \\
\hline CFHT/MegaPrime & $u^{\star}$ & SDSS $u$-band \\
& $g^{\star}$ & SDSS $g$-band \\
\hline & $J$ & NIR $J$-band \\
& $K_{\mathrm{S}}$ & NIR -band \\
\hline
\end{tabular}

In general, each cluster was observed deeply in at least three to six bands in the optical, with exposure times ranging from 1000 to $10,000 \mathrm{~s}$ per passband. Typical seeing in the $R_{\mathrm{C}}$ band, mostly used for weak-lensing shape measurements, is around 0 '.6-0'.8. Typical limiting magnitudes are $\sim 26-26.5$ in the $R_{\mathrm{C}}$ band for a $3 \sigma$ detection within a $2^{\prime \prime}$ diameter aperture (Umetsu et al. 2012; Medezinski et al. 2013).

Basic information regarding the weak-lensing band is given in Table 1 . In the present analysis we use the $R_{\mathrm{C}}$ band $\left(R_{844}\right.$ for RX J2248.7-4431) to measure the shapes of background galaxies for all clusters except A383 (Zitrin et al. 2011) and MACS J1206.2-0847 (Umetsu et al. 2012), both of which are based on our published CLASH lensing work. Zitrin et al. (2011) and Umetsu et al. (2012) used the $i^{\prime}$ - and $I_{\mathrm{C}}$-band images, respectively, for their weak-lensing shape analyses because these are of higher quality than the respective $R_{\mathrm{C}}$ band in terms of the stability and coherence of the point-spread function (PSF) anisotropy pattern, taken in fairly good seeing conditions.

Details regarding our reduction and analysis pipelines can be found in Umetsu et al. (2012) and Medezinski et al. (2013). We thus refer the reader to those papers and give a basic summary here. Our reduction pipeline derives from Nonino et al. (2009) and has been optimized separately for accurate photometry and weak-lensing shape measurements.

For photometric measurements, standard reduction steps include bias subtraction, super-flat-field correction, and PSF matching between exposures in the same band. An accurate astrometric solution is derived with the SCAMP software (Bertin 2006), using the Two Micron All Sky Survey ${ }^{28}$ as an external reference catalog, or the Sloan Digital Sky Survey (SDSS; Adelman-McCarthy et al. 2008) where available. ${ }^{29}$ Finally, the SwarP software (Bertin et al. 2002) is utilized to

\footnotetext{
28 This publication makes use of data products from the Two Micron All Sky Survey, which is a joint project of the University of Massachusetts and the Infrared Processing and Analysis Center/California Institute of Technology, funded by the National Aeronautics and Space Administration and the National Science Foundation.

29 This research has made use of the VizieR catalog access tool, CDS, Strasbourg, France.
} 
stack the single exposures on a common World Coordinate System grid with pixel scale of $0^{\prime \prime} 2$.

The photometric zero points for the co-added images were derived using HST/ACS magnitudes of cluster elliptical-type galaxies. These zero points were further refined by fitting spectral energy distribution templates with the BPZ code (Bayesian photometric redshift estimation; Benítez 2000; Benítez et al. 2004) to galaxies having spectroscopic redshifts where available. This leads to a final photometric accuracy of $\sim 0.01 \mathrm{mag}$ in all passbands. The magnitudes were corrected for Galactic extinction according to Schlegel et al. (1998). The multi-band photometry was measured using SExtractor (Bertin \& Arnouts 1996) in dual-image mode on PSF-matched images created by ColorPro (Coe et al. 2006).

For weak-lensing shape measurements, we separately stack data collected at different epochs and different camera rotation angles. We do not smear the single exposures before stacking, so as not to degrade the weak-lensing information derived from the shapes of galaxies. A shape catalog is created for each epoch and camera rotation separately. Then, these subcatalogs are combined by properly weighting and stacking the calibrated distortion measurements for galaxies in the overlapping region (see Section 4.3).

\subsection{Shape Measurement}

For shape measurements of background galaxies, we use our well-tested weak-lensing pipeline based on the IMCAT package (Kaiser et al. 1995, KSB) incorporating improvements developed in Umetsu et al. (2010). Our KSB+ implementation has been applied extensively to Subaru observations of a large number of clusters (e.g., Umetsu \& Broadhurst 2008; Okabe \& Umetsu 2008; Umetsu et al. 2009, 2010, 2011a, 2012; Medezinski et al. 2010, 2011, 2013; Coe et al. 2012).

Full details of our CLASH weak-lensing shape analysis pipeline are given in Umetsu et al. (2012). Here, we only highlight several aspects of our weak-lensing analysis pipeline.

1. Object detection. Objects are detected using the IMCAT peak-finding algorithm hfindpeaks, which for each object yields object parameters such as the peak position, Gaussian scale length, $r_{g}$, and significance of the peak detection, $v$.

2. Rejection of close pairs. Objects having any detectable neighbor within $3 r_{g}$ are identified. All such close pairs of objects are rejected to avoid possible shape measurement errors due to crowding. The detection threshold is set to $v=7$ for close-pair identification. After this close-pair rejection, objects with low detection significance $v<10$ are excluded from our analysis.

3. Shear calibration. Following Umetsu et al. (2010), we calibrate KSB's isotropic correction factor $P_{g}$ as a function of $r_{g}$ and magnitude, using galaxies detected with high significance $v>30$. This is to minimize the inherent shear calibration bias in the presence of measurement noise (Okura \& Futamase 2012).

4. Combining subcatalogs. For each galaxy, we combine shape measurements and associated errors from different epochs and camera orientations by $g=$ $\left(\sum_{k=1}^{N_{\text {sub }}} w_{k} g_{k}\right) /\left(\sum_{k=1}^{N_{\text {sub }}} w_{k}\right)$ and $\sigma_{g}^{2}=\left(\sum_{k=1}^{N \text { sub }} w_{k} \sigma_{g, k}^{2}\right) /$ $\left(\sum_{k=1}^{N_{\text {sub }}} w_{k}\right)$, where $N_{\text {sub }}$ is the number of subcatalogs, $g_{k}$ is the complex reduced-shear estimate for the galaxy in the $k$ th subcatalog, and $w_{k}$ is its statistical weight, defined such that $w_{k}=\left(\sigma_{g, k}^{2}+\alpha_{g}^{2}\right)^{-1}$ if the galaxy exists in the $k$ th subcatalog, and $w_{k}=0$ otherwise. Here $\alpha_{g}^{2}$ is the softening constant variance, taken to be $\alpha_{g}=0.4$ (e.g., Umetsu \& Broadhurst 2008; Umetsu et al. 2009, 2012; Okabe et al. 2010).

On the basis of simulated Subaru/Suprime-Cam images (Oguri et al. 2012; Massey et al. 2007), we found in Umetsu et al. (2010) that the lensing signal can be recovered with $|m| \sim 5 \%$ of the multiplicative shear calibration bias (as defined by Heymans et al. 2006; Massey et al. 2007) and $c \sim 10^{-3}$ of the residual shear offset, which is about one order of magnitude smaller than the typical distortion signal in cluster outskirts. Accordingly, we include for each galaxy a shear calibration factor of $1 / 0.95$ to account for residual calibration. ${ }^{30}$

\subsection{Background Galaxy Selection}

In general, the attainable number density $\bar{n}_{g}$ of background galaxies for use in weak-lensing shape measurements is sensitive to the image quality (seeing FWHM) and depth of observations. In the shot-noise-limited regime, the statistical precision of the weak-lensing measurements scales as $1 / \sqrt{\bar{n}_{g}}$. However, a careful background selection is even more critical for a cluster weak-lensing analysis, so that unlensed cluster members and foreground galaxies do not dilute the true lensing signal of the background. In particular, this lensing dilution effect due to contamination by cluster members can lead to a substantial underestimation of the true signal for $R \lesssim 400 \mathrm{kpc} h^{-1}$ by a factor of two to five, as demonstrated in Broadhurst et al. (2005) and Medezinski et al. (2010). The relative importance of the dilution effect indicates that the impact of background purity and depth is more important than that of shot noise $\left(\propto \bar{n}_{g}^{-1 / 2}\right)$.

We use the color-color (CC) selection method of Medezinski et al. (2010) to define undiluted samples of background galaxies from which to measure the weak-lensing shear and magnification effects. Here we refer the reader to Medezinski et al. (2010) for further details. This method is designed to avoid the inclusion of unlensed cluster members and to minimize foreground contamination on the basis of empirical correlations in CC-magnitude space, which have been established by reference to evolutionary color tracks of galaxies (Medezinski et al. 2010, 2011; Hanami et al. 2012), as well as to the 30-band photo- $z$ distribution in the COSMOS field (Ilbert et al. 2009). Using CCselected samples of differing depths, we showed in Medezinski et al. (2011) that the redshift scaling of the observed shear signal behind massive clusters follows the expected form of the lensing distance-redshift relation $\beta(z)$, providing independent consistency checks. Our color-cut approach and its variants have been successfully applied to a large number of clusters (Medezinski et al. 2010, 2011, 2013; Umetsu et al. 2010, 2011a, 2012; Coe et al. 2012; Oguri et al. 2012; Covone et al. 2014).

In the present analysis, we typically use the Subaru $B_{\mathrm{J}} R_{\mathrm{C}} z^{\prime}$ photometry where available, which spans the full optical wavelength range to perform CC selection of background samples. The specific CC-selection bands used for each cluster are indicated in Table 1. For shape measurements, we select and combine two distinct populations that encompass the red and blue branches of background galaxies in CC space (Medezinski et al. 2010, 2013; Umetsu et al. 2010, 2012), each with typical redshift distributions peaked around $z \sim 1$ and $\sim 2$, respectively (see Figures 5 and 6 of Medezinski et al. 2011; Lilly et al. 2007).

\footnotetext{
30 Our earlier CLASH weak-lensing work of Zitrin et al. (2011, A383), Umetsu et al. (2012, MACS J1206.2-0847), and Coe et al. (2012, A2261) did not include the $5 \%$ residual correction.
} 
Table 3

Background Galaxy Samples for Weak-lensing Shape Measurements

\begin{tabular}{|c|c|c|c|c|c|}
\hline Cluster & $N_{g}$ & $\bar{n}_{g}^{\mathrm{a}}$ & $\bar{z}_{\text {eff }}{ }^{b}$ & $\left\langle D_{\mathrm{ls}} / D_{\mathrm{s}}\right\rangle$ & $f_{W}$ \\
\hline \multicolumn{6}{|c|}{ X-ray selected } \\
\hline A383 & 7062 & 9.3 & 1.16 & $0.79 \pm 0.04$ & 1.01 \\
\hline A209 & 14694 & 15.8 & 0.94 & $0.74 \pm 0.04$ & 1.04 \\
\hline A2261 & 15429 & 18.1 & 0.88 & $0.70 \pm 0.04$ & 1.05 \\
\hline RX J2129.7+0005 & 20104 & 21.1 & 1.16 & $0.75 \pm 0.04$ & 1.02 \\
\hline A611 & 7872 & 8.5 & 1.13 & $0.68 \pm 0.03$ & 1.07 \\
\hline MS2137-2353 & 9864 & 10.3 & 1.23 & $0.68 \pm 0.03$ & 1.02 \\
\hline RX J2248.7-4431 & 4008 & 4.6 & 1.05 & $0.60 \pm 0.03$ & 1.15 \\
\hline MACS J1115.9+0129 & 13621 & 12.7 & 1.15 & $0.63 \pm 0.03$ & 1.03 \\
\hline MACS J1931.8-2635 & 4343 & 4.9 & 0.93 & $0.56 \pm 0.03$ & 1.06 \\
\hline RX J1532.9+3021 & 13270 & 16.2 & 1.15 & $0.61 \pm 0.03$ & 1.05 \\
\hline MACS J1720.3+3536 & 9855 & 12.0 & 1.12 & $0.58 \pm 0.03$ & 1.04 \\
\hline MACS J0429.6-0253 & 9990 & 11.7 & 1.30 & $0.62 \pm 0.06$ & 1.04 \\
\hline MACS J1206.2-0847 & 12719 & 13.3 & 1.13 & $0.54 \pm 0.03$ & 1.06 \\
\hline MACS J0329.7-0211 & 25427 & 29.3 & 1.18 & $0.54 \pm 0.03$ & 1.06 \\
\hline RXJ1347.5-1145 & 9393 & 7.9 & 1.17 & $0.54 \pm 0.03$ & 1.06 \\
\hline MACS J0744.9+3927 & 7561 & 8.0 & 1.41 & $0.42 \pm 0.02$ & 1.15 \\
\hline \multicolumn{6}{|c|}{ High magnification } \\
\hline MACS J0416.1-2403 & 21241 & 24.9 & 1.24 & $0.61 \pm 0.03$ & 1.01 \\
\hline MACS J1149.5+2223 & 14016 & 14.2 & 1.04 & $0.41 \pm 0.02$ & 1.24 \\
\hline MACS J0717.5+3745 & 9724 & 11.1 & 1.26 & $0.48 \pm 0.02$ & 1.09 \\
\hline MACS J0647.7+7015 & 7339 & 10.2 & 1.27 & $0.45 \pm 0.02$ & 1.09 \\
\hline
\end{tabular}

Notes.

a Mean surface number density of background galaxies per $\operatorname{arcmin}^{2}$.

b Effective source redshift corresponding to the mean lensing depth $\langle\beta\rangle=$ $\left\langle D_{\mathrm{ls}} / D_{\mathrm{s}}\right\rangle$ of the sample, defined as $\beta\left(\bar{z}_{\mathrm{eff}}\right)=\langle\beta\rangle$.
Our conservative selection criteria yield a typical (median) surface number density of $\bar{n}_{g} \simeq 12$ galaxies $\operatorname{arcmin}^{-2}$ for the weak-lensing-matched background catalogs (Table 3), consistent with the values found by Oguri et al. (2012). For RX J2248.7-4431 based on the $2.2 \mathrm{~m} / \mathrm{WFI}$ data, we have $\bar{n}_{g}=4.6$ galaxies $\operatorname{arcmin}^{-2}$, which is about a factor of 2.5 smaller than the median value of our sample. That is, the shotnoise level for the cluster is about $40 \%$ higher than that obtained with the typical depth of our Subaru observations. Accordingly, our weak-lensing measurements of RX J2248.7-4431 are highly shot-noise limited. On the other hand, the low number density of background galaxies in the MACS J1931.8-2635 field, $\bar{n}_{g}=4.9$ galaxies arcmin ${ }^{-2}$, is due to its low Galactic latitude, $b=-20.09$, which implies a high stellar density and a correspondingly large area masked by bright saturated stars. Our magnification-bias measurements are based on the flux-limited samples of red background galaxies at $\langle z\rangle \sim 1$, with a typical count slope of $\left\langle s_{\text {eff }}\right\rangle \sim 0.15$ (see Table 4 ).

We estimate the respective depths $\langle\beta\rangle$ and $\left\langle\beta^{2}\right\rangle$ of the different galaxy samples (Tables 3 and 4 ), when converting the observed lensing signal into physical mass units. For this we utilize BPZ to measure photo- $z$ 's $\left(z_{\text {phot }}\right)$ using our deep PSF-corrected multi-band photometry. Following Umetsu et al. (2012), we employ BPZ's ODDS parameter as the weight factor $w(z)$ in Equation (5).

We discard galaxies having photo-z's in the range $z_{\text {phot }}>2.5$ and having ODDS $<0.8$, as we find those to be spurious and unreliable. We derive this scheme by comparing our photo- $z$ 's with measured spectroscopic redshifts $\left(z_{\text {spec }}\right)$, compiled from

Table 4

Background Galaxy Samples for Magnification Bias Measurements

\begin{tabular}{|c|c|c|c|c|c|c|}
\hline Cluster & $\begin{array}{c}m_{\lim }{ }^{\mathrm{a}} \\
(\mathrm{AB} \text { mag) }\end{array}$ & $N_{\mu}$ & $\begin{array}{c}\bar{n}_{\mu}^{\mathrm{b}} \\
\left(\operatorname{arcmin}^{-2}\right)\end{array}$ & $s_{\text {eff }}{ }^{c}$ & $\bar{z}_{\text {eff }}{ }^{\mathrm{d}}$ & $\left\langle D_{\mathrm{ls}} / D_{\mathrm{s}}\right\rangle$ \\
\hline \multicolumn{7}{|c|}{ X-ray selected } \\
\hline A383 & 25.2 & 13763 & $13.3 \pm 0.4$ & $0.14 \pm 0.05$ & 1.23 & $0.80 \pm 0.04$ \\
\hline A209 & 25.1 & 12860 & $13.1 \pm 0.4$ & $0.14 \pm 0.05$ & 1.03 & $0.75 \pm 0.04$ \\
\hline A2261 & 25.6 & 17610 & $20.0 \pm 0.4$ & $0.14 \pm 0.04$ & 1.26 & $0.77 \pm 0.04$ \\
\hline RX J2129.7+0005 & 25.6 & 13467 & $13.9 \pm 0.5$ & $0.19 \pm 0.05$ & 1.05 & $0.73 \pm 0.04$ \\
\hline A611 & 25.6 & 7982 & $9.7 \pm 0.5$ & $0.20 \pm 0.06$ & 1.16 & $0.69 \pm 0.03$ \\
\hline MS2137-2353 & 25.6 & 18095 & $17.2 \pm 1.5$ & $0.13 \pm 0.04$ & 1.05 & $0.64 \pm 0.03$ \\
\hline RX J2248.7-4431 & 24.1 & 2685 & $4.4 \pm 0.3$ & $0.11 \pm 0.11$ & 1.18 & $0.64 \pm 0.03$ \\
\hline MACS J1115.9+0129 & 24.9 & 13109 & $14.0 \pm 0.5$ & $0.14 \pm 0.05$ & 0.98 & $0.58 \pm 0.03$ \\
\hline MACS J1931.8-2635 & 24.1 & 5556 & $6.1 \pm 0.4$ & $0.20 \pm 0.07$ & 0.90 & $0.55 \pm 0.03$ \\
\hline RX J1532.9+3021 & 25.4 & 18653 & $14.3 \pm 0.5$ & $0.17 \pm 0.04$ & 0.99 & $0.57 \pm 0.03$ \\
\hline MACS J1720.3+3536 & 25.2 & 17804 & $16.3 \pm 0.6$ & $0.16 \pm 0.04$ & 1.05 & $0.56 \pm 0.03$ \\
\hline MACS J0429.6-0253 & 25.4 & 13521 & $12.3 \pm 0.4$ & $0.19 \pm 0.05$ & 1.05 & $0.55 \pm 0.06$ \\
\hline MACS J1206.2-0847 & 24.6 & 13252 & $11.4 \pm 0.4$ & $0.13 \pm 0.05$ & 1.04 & $0.51 \pm 0.03$ \\
\hline MACS J0329.7-0211 & 25.4 & 21192 & $22.1 \pm 0.5$ & $0.13 \pm 0.04$ & 1.01 & $0.49 \pm 0.02$ \\
\hline RX J1347.5-1145 & 25.6 & 15017 & $14.2 \pm 0.5$ & $0.14 \pm 0.04$ & 1.04 & $0.50 \pm 0.02$ \\
\hline MACS J0744.9+3927 & 25.6 & 17165 & $15.5 \pm 0.5$ & $0.15 \pm 0.04$ & 1.23 & $0.36 \pm 0.02$ \\
\hline \multicolumn{7}{|c|}{ High magnification } \\
\hline MACS J0416.1-2403 & 25.6 & 27068 & $27.2 \pm 0.6$ & $0.17 \pm 0.03$ & 1.07 & $0.56 \pm 0.03$ \\
\hline MACS J1149.5+2223 & 25.6 & 19271 & $18.3 \pm 0.6$ & $0.15 \pm 0.04$ & 1.06 & $0.41 \pm 0.02$ \\
\hline MACS J0717.5+3745 & 25.6 & 11641 & $11.9 \pm 0.4$ & $0.12 \pm 0.05$ & 1.15 & $0.45 \pm 0.02$ \\
\hline MACS J0647.7+7015 & 25.6 & 15043 & $14.3 \pm 0.5$ & $0.13 \pm 0.04$ & 1.17 & $0.42 \pm 0.02$ \\
\hline
\end{tabular}

Notes.

${ }^{a}$ Fainter magnitude cut of the background sample. Apparent magnitude cuts are applied in the reddest CC-selection band available for each cluster to avoid incompleteness near the detection limit.

${ }^{\mathrm{b}}$ Coverage- and mask-corrected normalization of unlensed background source counts.

${ }^{\mathrm{c}}$ Slope of the unlensed background source counts $s_{\text {eff }}=d \log _{10} \bar{N}_{\mu}(<m) / d m$.

${ }^{\mathrm{d}}$ Effective source redshift corresponding to the mean lensing depth of the sample, defined as $\beta\left(\bar{z}_{\text {eff }}\right)=\langle\beta\rangle$. 
both the NASA/IPAC Extragalactic Database (NED) ${ }^{31}$ and our VLT-CLASH large spectroscopic program (ID: 186.A-0798; PI: P. Rosati). We find that when using the full photo- $z$ catalog, although we obtain a reasonable accuracy with a normalized median absolute deviation (NMAD) of 1.48 MAD $\left(\left(z_{\mathrm{phot}}-\right.\right.$ $\left.\left.z_{\text {spec }}\right) /\left(1+z_{\text {spec }}\right)\right)=3.1 \%$, the outlier fraction is high, $f_{\text {outliers }}=$ $14 \%$, where the outliers are defined as galaxies with $\mid z_{\text {phot }}-$ $z_{\text {spec }} \mid /\left(1+z_{\text {spec }}\right)>0.15$ (Jouvel et al. 2014). These outliers mostly stem from galaxies identified to have $z_{\text {phot }}>2.5$, whereas their true redshifts are low, $z_{\text {spec }}<0.6$. Excluding galaxies with $z_{\text {phot }}>2.5$, we find an NMAD of $2.7 \%$ and an outlier fraction of $f_{\text {outlier }}=9.5 \%$.

For a consistency check, we also make use of the COSMOS catalog (Ilbert et al. 2009) with robust photometry and photo- $z$ measurements for the majority of galaxies with $i^{\prime}<25 \mathrm{mag}$. For each cluster, we apply the same CC selection to the COSMOS photometry and obtain the redshift distribution $\bar{N}(z)$ of field galaxies. Since COSMOS is only complete to $i^{\prime}<25 \mathrm{mag}$, we derive the mean depth $\langle\beta\rangle$ as a function of magnitude up to that limit and extrapolate to our limiting magnitudes (Medezinski et al. 2013, see their Section 3.3). For our sample of 20 CLASH fields, we find an excellent statistical agreement between the BPZ- and COSMOS-based depth estimates $\langle\beta\rangle$, with a median relative offset of $0.27 \%$ and an rms cluster-to-cluster scatter of $5.0 \%$.

\section{CLASH SHEAR-AND-MAGNIFICATION ANALYSIS}

In this section we carry out a joint shear-and-magnification analysis of a sample of 20 CLASH clusters. In Figure 1 we present our weak-lensing distortion data for our sample in the form of two-dimensional mass maps, where we have used the linear mapmaking method outlined in Umetsu et al. (2009). The mass maps are smoothed with a Gaussian with 1'.8 FWHM and presented primarily for visualization purposes.

\subsection{Cluster Center}

As summarized in Table 1, our cluster sample exhibits on average a small offset $d_{\text {off }}=\left|\boldsymbol{d}_{\text {off }}\right|$ between the BCG and X-ray peak, characterized by a median offset of $\bar{d}_{\text {off }} \simeq 10 \mathrm{kpc} h^{-1}$ and an rms offset of $\sigma_{\text {off }} \simeq 30 \mathrm{kpc} h^{-1}$. For the X-ray-selected subsample, we find a much smaller rms of $\sigma_{\text {off }} \simeq 11 \mathrm{kpc} h^{-1}$. Johnston et al. (2007) demonstrated that $\kappa$ is much less affected by cluster miscentering than $\gamma_{+}$, and that the miscentering effects on $\kappa$ nearly vanish at twice the typical positional offset from the cluster mass centroid. This indicates that our massprofile reconstructions would not be affected, on average, by the miscentering effects beyond a radius of $R \sim 60 \mathrm{kpc} h^{-1}$ ( $R \sim 20 \mathrm{kpc} h^{-1}$ for the X-ray-selected subsample). This level of centering offset is much smaller than the range of overdensity radii of interest for cluster mass measurements, and hence will not significantly affect our cluster mass profile measurements. ${ }^{32}$ In what follows, we will adopt the BCG position as the cluster center (Table 1).

\subsection{Cluster Mass Profiles}

First, we derive azimuthally averaged lens distortion and count depletion profiles (Section 3) for our cluster sample from

\footnotetext{
31 http://ned.ipac.caltech.edu/

32 This level of offset could potentially lead to an underestimation of the central cusp slope, which, however, is beyond the scope of this weak-lensing analysis.
}

Table 5

Cluster Weak-lensing Radial Profiles

\begin{tabular}{|c|c|c|c|}
\hline \multirow[t]{2}{*}{ Cluster } & \multicolumn{2}{|c|}{$\mathrm{S} / \mathrm{N}$} & \multirow[t]{2}{*}{$\chi_{\min }^{2}{ }^{a}$} \\
\hline & $g_{+}$ & $n_{\mu}$ & \\
\hline \multicolumn{4}{|c|}{ X-ray selected } \\
\hline A383 & 9.2 & 3.1 & 5.8 \\
\hline A209 & 14.1 & 3.0 & 5.7 \\
\hline A2261 & 16.4 & 8.1 & 13.8 \\
\hline RX J2129.7+0005 & 10.3 & 2.5 & 9.1 \\
\hline A611 & 7.7 & 2.8 & 2.2 \\
\hline MS2137-2353 & 8.5 & 4.0 & 7.5 \\
\hline RX J2248.7-4431 & 6.9 & 4.3 & 5.1 \\
\hline MACS J1115.9+0129 & 9.2 & 3.9 & 4.5 \\
\hline MACS J1931.8-2635 & 4.8 & 3.8 & 8.0 \\
\hline RX J1532.9+3021 & 6.6 & 5.0 & 14.3 \\
\hline MACS J1720.3+3536 & 7.7 & 4.4 & 9.6 \\
\hline MACS J0429.6-0253 & 7.7 & 4.0 & 14.4 \\
\hline MACS J1206.2-0847 & 9.5 & 4.2 & 5.8 \\
\hline MACS J0329.7-0211 & 12.9 & 4.3 & 9.6 \\
\hline RXJ1347.5-1145 & 9.7 & 5.8 & 8.1 \\
\hline MACS J0744.9+3927 & 8.7 & 3.6 & 10.5 \\
\hline \multicolumn{4}{|c|}{ High magnification } \\
\hline MACS J0416.1-2403 & 10.7 & 3.8 & 9.6 \\
\hline MACS J1149.5+2223 & 9.6 & 2.4 & 5.7 \\
\hline MACS J0717.5+3745 & 12.3 & 5.5 & 6.1 \\
\hline MACS J0647.7+7015 & 6.8 & 4.3 & 11.1 \\
\hline
\end{tabular}

Notes. The lensing radial profiles are calculated in $N=10$ discrete radial bins over the radial range of $\left[\theta_{\min }, \theta_{\max }\right]$, with a logarithmic radial spacing of $\Delta \ln \theta=\ln \left(\theta_{\max } / \theta_{\min }\right) / N$. For all clusters, $\theta_{\min }=0^{\prime} .9$ and $\theta_{\max }=16^{\prime}$, except $\theta_{\max }=14^{\prime}$ for RX J2248.7-4431 observed with ESO/WFI.

${ }^{\text {a }}$ Minimum $\chi^{2}$ obtained for the mass-profile solution. For all clusters, the number of degrees of freedom is $2 N-(N+1)=9$.

our wide-field imaging data (Section 4). The radial binning scheme is summarized in Table 5.

For each cluster we calculate the lensing profiles in $N$ discrete radial bins from the cluster center (Table 1), spanning the range $\left[\theta_{\min }, \theta_{\max }\right]$ with a constant logarithmic radial spacing $\Delta \ln \theta=\ln \left(\theta_{\max } / \theta_{\min }\right) / N$, where the inner radial boundary $\theta_{\min }$ is taken for all clusters to be $\theta_{\min }=0.9$, which is larger than the range of Einstein radii for our sample (A. Zitrin et al. 2014, in preparation). For all clusters in our sample, the inner radial limits satisfy $D_{1} \theta_{\text {min }}>2 d_{\text {off }}$, so that the miscentering effects on our mass profile measurements are negligible (Johnston et al. 2007; Umetsu et al. 2011b). The choice of $\theta_{\min }$ also ensures high-purity samples of background galaxies with a low level of contamination by cluster members defined by our CC-selection method (Section 4.4). The outer boundary is set to $\theta_{\max }=16^{\prime}$ (Umetsu et al. 2012; Coe et al. 2012; Medezinski et al. 2013) for all clusters, except RX J2248.7-4431 observed with ESO/ WFI, for which we take $\theta_{\max }=14^{\prime}$. These are sufficiently larger than the range of virial radii for our sample $\left(r_{\mathrm{vir}} \lesssim 2 \mathrm{Mpc} h^{-1}\right)$, but sufficiently small with respect to the sizes of the camera field of view so as to ensure accurate PSF anisotropy correction. The number of radial bins is set to $N=10$ for all clusters, such that the per-pixel detection $\mathrm{S} / \mathrm{N}$ is of the order of unity, which is optimal for an inversion problem. Here we quantify the significance of a detection for a given lensing profile in analogy to Equation (38) of Umetsu \& Broadhurst (2008).

Unlike the distortion effect, the magnification signal falls off sharply with increasing distance from the cluster center. For the 

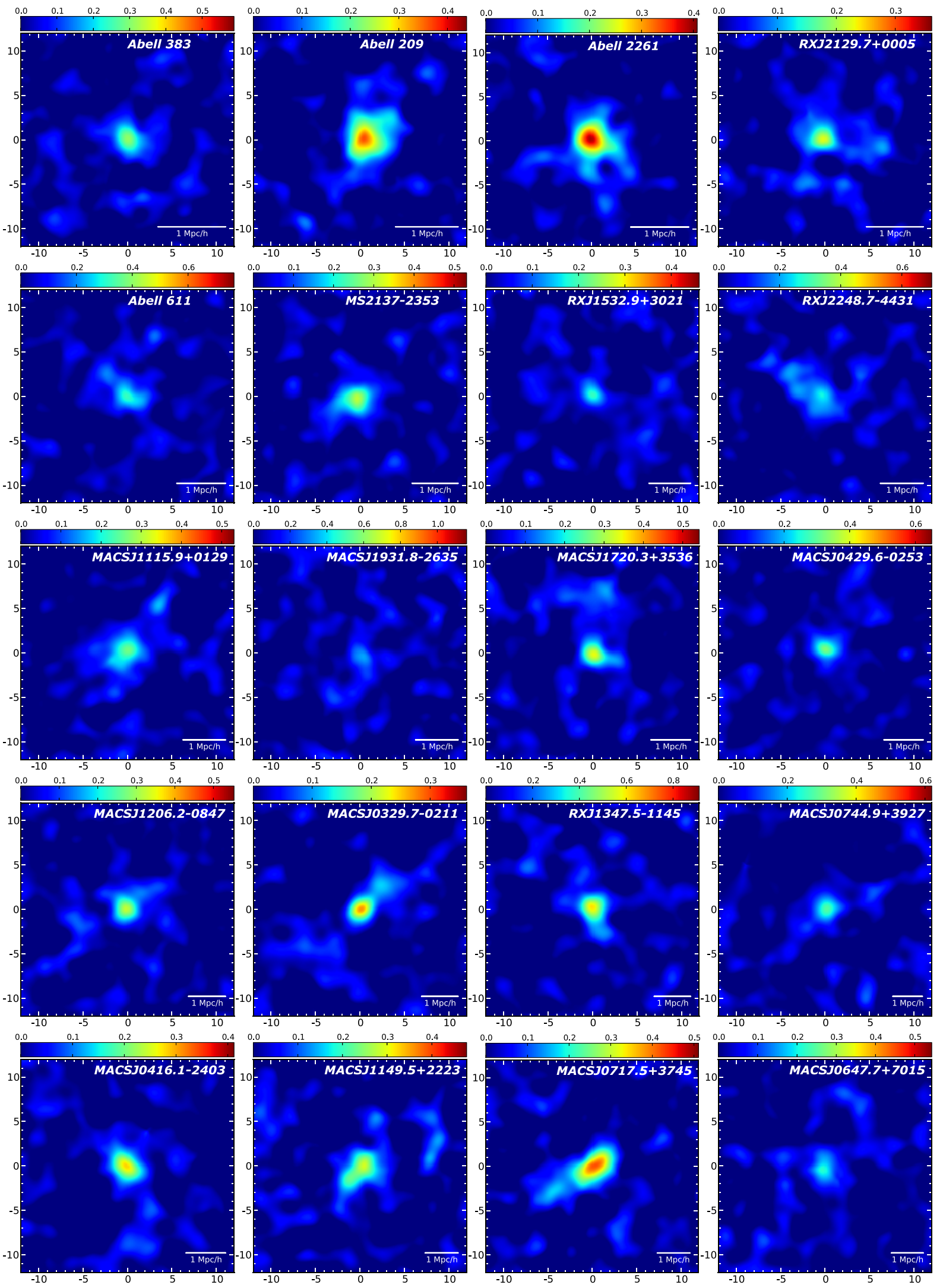

Figure 1. Two-dimensional weak-lensing mass maps $\Sigma(\boldsymbol{\theta})$ for a sample of 20 CLASH clusters reconstructed using wide-field multi-color imaging observations. For visualization purposes, all images are smoothed with a circular Gaussian of FWHM 1'.8. The images are $24^{\prime} \times 24^{\prime}$ in size and centered on the respective optical cluster centers. The color bar indicates the lensing convergence $\kappa=\Sigma / \Sigma_{\mathrm{c}}$, the surface mass density $\Sigma$ in units of the critical surface mass density $\Sigma_{\mathrm{c}}$. The color scale is linear from $\mathrm{S} / \mathrm{N} \equiv \kappa / \sigma_{\kappa}$ of 0 (dark blue) to 15 (dark red) for all clusters. North is to the top, east to the left. The horizontal bar in each panel represents $1 \mathrm{Mpc} h^{-1}$ at the cluster redshift.

(A color version of this figure is available in the online journal.) 
magnification-bias analysis, the count normalization and slope $\left(\bar{n}_{\mu}, s_{\text {eff }}\right)$ are estimated from the source counts in cluster outskirts (Umetsu et al. 2011a, 2012; Coe et al. 2012; Medezinski et al. 2013), specifically at $\theta=\left[10^{\prime}, \theta_{\max }\right]$ (see Section 7.4.2).

In Figure 2 we show the distortion and magnification profiles for our sample of 20 CLASH clusters. For most of the clusters, a systematic depletion of the red galaxy counts is seen in the high-density region of the cluster and detected out to several arcminutes from the cluster center. For our sample of 20 cluster fields, we find a median masked-area fraction $\bar{f}_{\text {mask }}$ (Section 3.2.2) of 0.076 with a standard deviation of 0.055 . The statistical significance of the detection of the tangential distortion ranges from $4.8 \sigma$ to $16.4 \sigma$. On the other hand, the detection significance of the depletion signal is in the range $2.5 \sigma-8.1 \sigma$ (Table 5), which is on average $45 \%$ of the $\mathrm{S} / \mathrm{N}$ of the tangential distortion. This corresponds to an overall improvement of $\sim 10 \%$ by combining the shear and magnification measurements, compared to the distortion-only case (Umetsu et al. 2011a, 2012; Umetsu 2013; Coe et al. 2012).

Following the methodology outlined in Section 3.3, we derive for each cluster a mass-profile solution $\Sigma=\left\{\Sigma_{\min }, \Sigma_{i}\right\}_{i=1}^{N}$ from a joint likelihood analysis of our shear+magnification data (Figure 2). In Table 5 we present the minimum $\chi^{2}(\equiv-2 \ln \mathcal{L})$ values for the best-fit $\boldsymbol{\Sigma}$ solutions, ranging from $\chi^{2}=2.2$ to 14.4 for $2 N-(N+1)=9$ degrees of freedom (a mean reduced $\chi^{2}$ of 0.92 ), indicating good consistency between the shear and magnification measurements having different systematics. This is also demonstrated in Figure 2, which compares the observed lensing profiles with the respective joint Bayesian reconstructions $(68 \% \mathrm{CL})$.

The resulting mass-profile solutions $\boldsymbol{\Sigma}$ are shown in Figure 3 for our 20 clusters along with their NFW fitting results (see Section 5.3). The error bars represent the $1 \sigma$ uncertainties from the diagonal part of the total covariance matrix $C=$ $C^{\text {stat }}+C^{\text {sys }}+C^{\text {lss }}$ (Equation (15)) including statistical, systematic, and projected uncorrelated LSS noise contributions.

\subsection{Cluster Mass Estimates}

The standard weak-lensing approach to cluster mass estimates is based on tangential-shear fitting with NFW functionals (Oguri et al. 2009; Okabe et al. 2010). This approach, however, has a disadvantage that the cluster mass estimates can be biased low by 5\%-10\% (Meneghetti et al. 2010b; Becker \& Kravtsov 2011; Rasia et al. 2012). This well-known bias arises from the fact that the tangential shear responds negatively to local mass perturbations (Equation (2)) that are abundant in rich cluster environments. This bias can be minimized if the fitting range is restricted to within $\sim 2 r_{500 \mathrm{c}}$ (Applegate et al. 2014). An alternative approach is to use the combination of shear and magnification to reconstruct the projected mass density profile, breaking the mass-sheet degeneracy.

Here we use our mass-profile data set (Figure 3 ) to obtain total mass estimates for individual clusters in our sample. To do this, we employ the spherical NFW model to facilitate comparison with results from complementary X-ray, Sunyaev-Zel'dovich effect (SZE), and dynamical observations. The two-parameter NFW profile is given by

$$
\rho_{\mathrm{NFW}}(r)=\frac{\rho_{\mathrm{s}}}{\left(r / r_{\mathrm{s}}\right)\left(1+r / r_{\mathrm{s}}\right)^{2}},
$$

where $\rho_{\mathrm{s}}$ and $r_{\mathrm{s}}$ are the characteristic density and radius, respectively. For the NFW model, $r_{\mathrm{s}}$ marks the radius at which the logarithmic density slope equals the isothermal value, namely, $d \ln \rho(r) / d \ln r=-2$ at $r=r_{\mathrm{s}}$. We specify the NFW model with the halo mass $M_{200 \mathrm{c}}$ and the corresponding halo concentration $c_{200 \mathrm{c}}=r_{200 \mathrm{c}} / r_{\mathrm{s}}$.

We use a Bayesian MCMC method to obtain an accurate inference of the NFW density profile (Equation (25)) from our data in the form of the discrete cluster mass profile $s=$ $\boldsymbol{\Sigma} / \Sigma_{\mathrm{c}, \infty}$ and its full covariance matrix $C=C^{\text {stat }}+C^{\text {sys }}+C^{\text {lss }}$ (Section 3.3). Here we employ the radial dependence of the projected NFW lensing profiles given by Wright \& Brainerd (2000), which provides a sufficiently good description of the projected total matter distribution in cluster-sized halos out to the virial radius $r_{\text {vir }}$ (Oguri \& Hamana 2011; Umetsu et al. 2011b; Oguri et al. 2012; Newman et al. 2013; Okabe et al. 2013; see also Section 3.2). For all clusters, we thus restrict the fitting range to $R \leqslant 2 \mathrm{Mpc} h^{-1}$, which is approximately the virial radius of high-mass clusters. We assume uninformative log-uniform priors for the mass and concentration parameters (i.e., uniform priors for $\log _{10} M_{200 c}$ and $\log _{10} c_{200 c}$; see Sereno \& Covone 2013; Covone et al. 2014) in the respective intervals, $0.1 \leqslant M_{200 \mathrm{c}} /\left(10^{15} M_{\odot} h^{-1}\right) \leqslant 10$ and $0.1 \leqslant c_{200 \mathrm{c}} \leqslant 10$. The $\chi^{2}$ function for our weak-lensing observations is

$$
\chi^{2}(\boldsymbol{p})=\sum_{i, j}\left[s_{i}-\hat{s}_{i}(\boldsymbol{p})\right] C_{i j}^{-1}\left[s_{j}-\hat{s}_{j}(\boldsymbol{p})\right],
$$

where $\boldsymbol{p}=\left(M_{200 \mathrm{c}}, c_{200 \mathrm{c}}\right)$, and $\hat{\boldsymbol{s}}(\boldsymbol{p})=\hat{\boldsymbol{\Sigma}}(\boldsymbol{p}) / \Sigma_{\mathrm{c}, \infty}$ is the model prediction for the binned mass profile, given as

$$
\hat{\Sigma}_{i}=\Sigma_{\mathrm{NFW}}\left(\theta_{i, 1}<\theta<\theta_{i, 2}\right),
$$

with $\Sigma_{\mathrm{NFW}}\left(\theta_{i, 1}<\theta<\theta_{i, 2}\right)$ the predicted mean surface mass density averaged over the $i$ th annulus between $\theta_{i, 1}$ and $\theta_{i, 2}$, accounting for the effect of bin averaging in observations.

From the posterior samples, we derive marginalized constraints on the total enclosed mass $M_{\Delta}=M_{3 \mathrm{D}}\left(<r_{\Delta}\right)$ at several characteristic interior overdensities $\Delta$ (see Section 1$)$. In Table 6 we summarize the results of our weak-lensing cluster mass estimates, where we employ the robust biweight estimators of Beers et al. (1990) for the central location (average) and scale (dispersion) of the marginalized posterior probability distributions (e.g., Sereno \& Umetsu 2011; Biviano et al. 2013).

\subsection{Systematic Mass Uncertainty \\ 5.4.1. Concentration-Mass Degeneracy}

A robust determination of the concentration parameter requires sensitive lensing measurements over a wide range of cluster radii because the concentration is sensitive to the radial curvature in the mass profile. In practice, such a measurement can be achieved by combining strong- and weak-lensing data (Merten et al. 2009), performing wide-field weak-lensing observations of nearby clusters (e.g., A2142 in Umetsu et al. 2009), or stacking the lensing signal of a statistical sample of clusters (Johnston et al. 2007; Umetsu et al. 2011b; Oguri et al. 2012).

In this work we have not attempted to determine the concentration for each cluster because the weak-lensing profiles for individual clusters are highly degenerate in $M$ and $c$ : the observed lensing profile can be explained by halo density profiles with larger $M$ and smaller $c$ than the best-fit values and vice versa. This $c-M$ degeneracy is more significant for low-mass, high-redshift systems, which are unresolved by weak-lensing observations and for which the scale radius $r_{\mathrm{s}}$ is unconstrained 

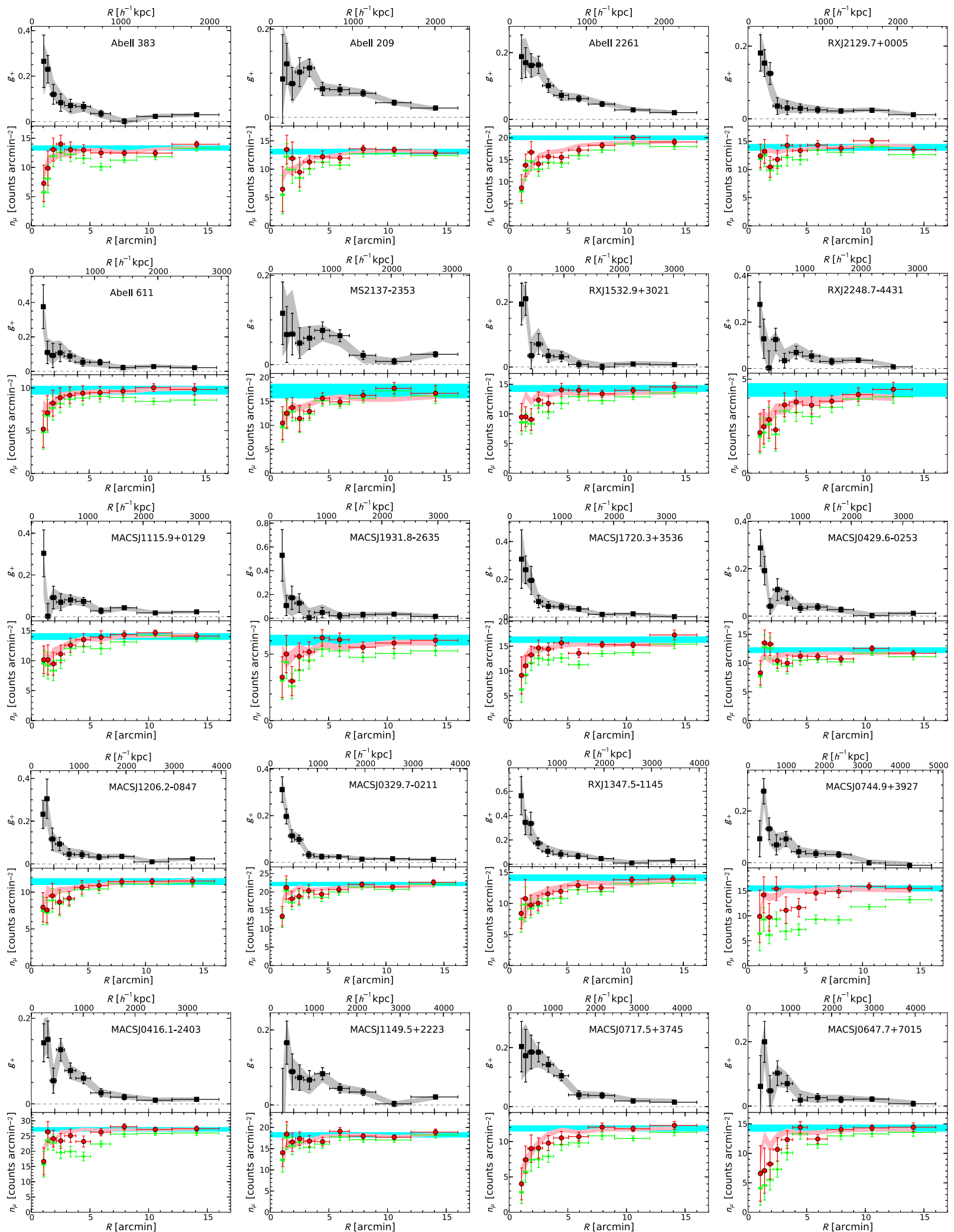

Figure 2. Azimuthally averaged radial profiles of the tangential-distortion $\left(g_{+}\right)$and magnification-bias $\left(n_{\mu}\right)$ measurements obtained from wide-field multi-color imaging observations, shown individually for our sample of 20 CLASH clusters. For each cluster, the upper panel shows the tangential reduced shear $g_{+}$(black squares) of background galaxies, and the lower panel shows the count-depletion profile $n_{\mu}$ of red background galaxies, with (red circles) and without (green dots) the mask correction due to bright foreground objects and cluster members. A systematic radial depletion of the source counts is seen toward the cluster center owing to magnification of the sky area. The error bars include contributions from Poisson counting uncertainties and contamination due to intrinsic angular clustering of red galaxies. For each observed profile, the shaded area represents the joint Bayesian reconstruction (68\% CL) from the combined tangential-distortion and magnification-bias measurements. The horizontal bar (cyan shaded region) shows the constraints on the unlensed count normalization $\bar{n}_{\mu}$ estimated from the source counts in cluster outskirts. A large correction for the incomplete area coverage, accounting for masked regions due to bright saturated stars, was applied to the number count profiles of low Galactic latitude clusters $\left(|b|<30^{\circ}\right)$, such as MACS J1931.8-2635 and MACS J0744.9+3927.

(A color version of this figure is available in the online journal.) 

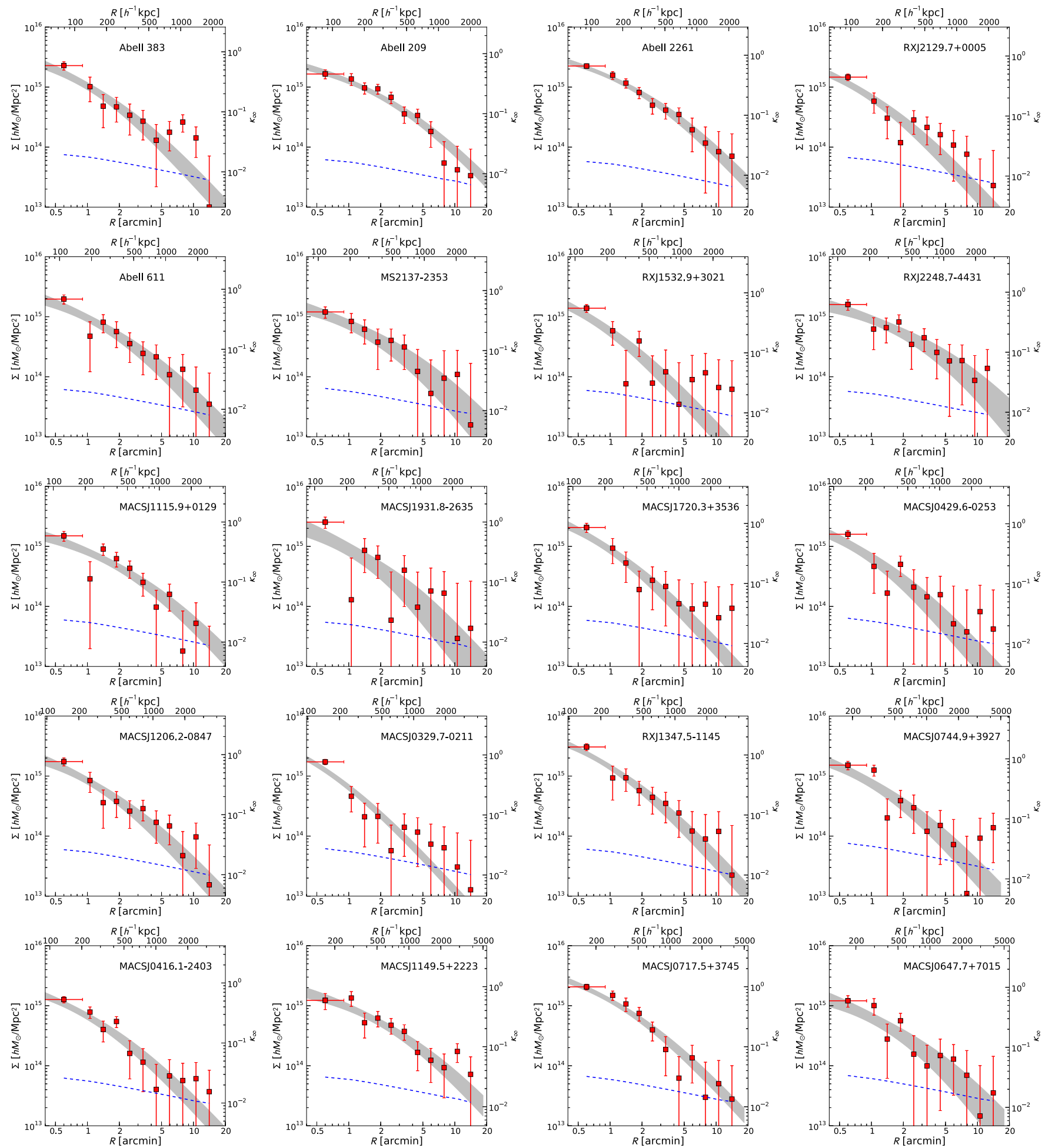

Figure 3. Cluster mass profile data set derived from our joint likelihood analysis of weak-lensing shear and magnification measurements shown in Figure 2. For each cluster, the central bin $\Sigma\left(<R_{\min }\right)$ is marked with a horizontal bar. The location of each binned $\Sigma$ point (squares) represents the area-weighted center of the radial band. The error bars represent the $1 \sigma$ uncertainty from the diagonal part of the total covariance matrix including statistical, systematic, and projected uncorrelated LSS contributions, $C=C^{\text {stat }}+C^{\text {sys }}+C^{\text {lss }}$. The gray area in each plot shows the best-fit NFW model $(68 \% \mathrm{CL})$ from the reconstructed $\Sigma$ profile. The dashed line shows the estimated contribution to the variance from uncorrelated LSS $C^{\mathrm{lss}}$ projected along the line of sight. The scale on the right vertical axis shows the corresponding lensing convergence $\kappa_{\infty}$ scaled to the reference far-background source plane.

(A color version of this figure is available in the online journal.)

by the data. In such a case, the constraints on $c$ are essentially imposed by prior information. This inherent covariance can potentially bias the slope of the $c(M)$ relation determined from weak lensing (Hoekstra et al. 2011).

\subsubsection{Impact of the Choice of the Fitting Range}

Unlike the shear, which is sensitive to the mean interior density, the majority of the $\kappa$ signal (with respect to the noise) 
Table 6

Cluster Mass Estimates Based on Joint Weak-lensing Shear+Magnification Measurements

\begin{tabular}{|c|c|c|c|c|c|c|}
\hline Cluster & $\begin{array}{c}M_{500 \mathrm{c}} \\
\left(10^{14} M_{\odot}\right)\end{array}$ & $\begin{array}{c}M_{200 c} \\
\left(10^{14} M_{\odot}\right)\end{array}$ & $\begin{array}{c}M_{\mathrm{vir}}{ }^{\mathrm{a}} \\
\left(10^{14} M_{\odot}\right)\end{array}$ & $\begin{array}{c}M_{100 \mathrm{c}} \\
\left(10^{14} M_{\odot}\right)\end{array}$ & $\begin{array}{c}M_{200 \mathrm{~m}} \\
\left(10^{14} M_{\odot}\right)\end{array}$ & $\begin{array}{c}M(<1.5 \mathrm{Mpc}) \\
\quad\left(10^{14} M_{\odot}\right)\end{array}$ \\
\hline \multicolumn{7}{|c|}{ X-ray selected } \\
\hline A383 & $6.1 \pm 1.5$ & $8.1 \pm 2.2$ & $9.4 \pm 2.8$ & $9.7 \pm 2.8$ & $10.3 \pm 3.1$ & $7.1 \pm 1.4$ \\
\hline A209 & $11.6 \pm 1.8$ & $17.6 \pm 3.0$ & $21.9 \pm 4.0$ & $22.8 \pm 4.2$ & $24.7 \pm 4.7$ & $11.6 \pm 1.2$ \\
\hline A2261 & $14.7 \pm 2.4$ & $21.3 \pm 4.1$ & $25.8 \pm 5.4$ & $26.8 \pm 5.8$ & $28.6 \pm 6.3$ & $13.7 \pm 1.5$ \\
\hline RX J2129.7+0005 & $4.1 \pm 0.9$ & $5.5 \pm 1.4$ & $6.4 \pm 1.7$ & $6.6 \pm 1.8$ & $6.9 \pm 1.9$ & $5.3 \pm 1.0$ \\
\hline A611 & $9.5 \pm 2.2$ & $14.1 \pm 3.9$ & $17.1 \pm 5.2$ & $18.1 \pm 5.7$ & $18.8 \pm 6.0$ & $10.3 \pm 1.7$ \\
\hline MS2137-2353 & $7.4 \pm 2.4$ & $12.4 \pm 4.8$ & $15.7 \pm 6.7$ & $17.0 \pm 7.5$ & $17.7 \pm 7.9$ & $9.0 \pm 2.0$ \\
\hline RX J2248.7-4431 & $11.1 \pm 3.2$ & $20.3 \pm 6.7$ & $26.7 \pm 9.9$ & $29.5 \pm 11.5$ & $30.2 \pm 12.0$ & $12.0 \pm 2.0$ \\
\hline MACS J1115.9+0129 & $9.3 \pm 2.0$ & $15.6 \pm 3.4$ & $19.6 \pm 4.5$ & $21.4 \pm 5.1$ & $21.8 \pm 5.2$ & $10.7 \pm 1.4$ \\
\hline MACS J1931.8-2635 & $10.2 \pm 3.9$ & $14.8 \pm 6.4$ & $17.4 \pm 8.2$ & $18.5 \pm 9.0$ & $18.8 \pm 9.2$ & $11.0 \pm 2.9$ \\
\hline RX J1532.9+3021 & $5.3 \pm 1.3$ & $7.1 \pm 1.9$ & $8.0 \pm 2.2$ & $8.4 \pm 2.4$ & $8.5 \pm 2.4$ & $6.6 \pm 1.3$ \\
\hline MACS J1720.3+3536 & $10.1 \pm 2.0$ & $13.5 \pm 3.1$ & $15.3 \pm 3.7$ & $16.2 \pm 4.0$ & $16.2 \pm 4.0$ & $11.0 \pm 1.7$ \\
\hline MACS J0429.6-0253 & $6.8 \pm 1.9$ & $9.4 \pm 3.0$ & $10.8 \pm 3.8$ & $11.5 \pm 4.2$ & $11.5 \pm 4.1$ & $8.3 \pm 1.8$ \\
\hline MACS J0329.7-0211 & $7.7 \pm 1.1$ & $10.0 \pm 1.5$ & $11.1 \pm 1.7$ & $11.7 \pm 1.8$ & $11.6 \pm 1.8$ & $9.0 \pm 1.0$ \\
\hline RXJ1347.5-1145 & $21.9 \pm 3.8$ & $29.5 \pm 6.1$ & $33.4 \pm 7.4$ & $35.6 \pm 8.2$ & $35.1 \pm 8.0$ & $19.7 \pm 2.3$ \\
\hline MACS J0744.9+3927 & $11.2 \pm 2.9$ & $17.5 \pm 4.7$ & $20.2 \pm 5.8$ & $23.1 \pm 7.0$ & $21.0 \pm 6.1$ & $13.5 \pm 2.3$ \\
\hline \multicolumn{7}{|c|}{ High magnification } \\
\hline MACS J0416.1-2403 & $7.0 \pm 1.3$ & $10.4 \pm 2.2$ & $12.4 \pm 2.8$ & $13.3 \pm 3.2$ & $13.3 \pm 3.1$ & $8.7 \pm 1.2$ \\
\hline MACS J1149.5+2223 & $14.2 \pm 3.4$ & $25.4 \pm 5.2$ & $31.5 \pm 6.5$ & $36.4 \pm 7.7$ & $34.0 \pm 7.1$ & $15.1 \pm 2.1$ \\
\hline MACS J0717.5+3745 & $20.9 \pm 2.9$ & $30.7 \pm 4.9$ & $35.3 \pm 6.0$ & $38.8 \pm 6.8$ & $37.1 \pm 6.4$ & $19.4 \pm 1.8$ \\
\hline MACS J0647.7+7015 & $7.7 \pm 2.7$ & $13.2 \pm 4.2$ & $16.0 \pm 5.3$ & $18.5 \pm 6.4$ & $17.0 \pm 5.8$ & $10.5 \pm 2.1$ \\
\hline
\end{tabular}

Notes. Cluster mass estimates $M_{3 \mathrm{D}}(<r)$ derived from single spherical NFW fits to individual projected mass density profiles (Figure 3 ) reconstructed from combined shear+magnification measurements. The fitting radial range is restricted to $R \leqslant 2 \mathrm{Mpc} h^{-1}$. All quantities in the table are given in physical units assuming a concordance cosmology of $h=0.7, \Omega_{\mathrm{m}}=0.27$, and $\Omega_{\Lambda}=0.73$. The results are subject to a systematic uncertainty of $\pm 8 \%$ in the overall mass calibration (Section 5.4.4).

${ }^{a}$ Virial overdensity $\Delta_{\text {vir }}$ based on the spherical collapse model (see Appendix A of Kitayama \& Suto 1996). For our redshift range $0.187 \leqslant z \leqslant 0.686$, $\Delta_{\text {vir }}$ ranges approximately from $\simeq 110$ to 140 with respect to the critical density of the universe at the cluster redshift.

comes from relatively inner regions and the outer profile exhibits a high degree of positive correlation ( $\sim 50 \%$ in the last few bins). The relative contribution of projected uncorrelated LSS noise increases with increasing radius (Figure 3), so that the effect of including $C^{\mathrm{lss}}$ is to further downweight the lensing signal in the outer regions especially beyond $\theta \sim 10^{\prime}$ (Hoekstra 2003). On average, we find that cosmic noise contributes $\sim 25 \%$ to the total error budget $\sqrt{C_{i i}}$ for the reconstructed $\kappa$ profile.

Without restricting the radial range for NFW fitting, we find $\sim 2 \%$ lower virial masses $\left(M_{\text {vir }}\right)$ relative to our baseline results obtained with a maximum fitting radius of $R=2 \mathrm{Mpc} h^{-1}$. This effect is less significant at higher overdensities, $\Delta_{\mathrm{c}} \geqslant 200$. We thus conclude that our cluster mass estimates $M_{\Delta}$ are statistically insensitive to the choice of the outer fitting radius.

\subsubsection{Halo Triaxiality}

Lensing mass measurements are sensitive to the halo triaxiality (Oguri et al. 2005). In the context of $\Lambda \mathrm{CDM}$, prolate halo shapes are expected to develop along filaments at early stages of halo assembly, so that dynamically young cluster halos tend to have a prolate morphology (Shaw et al. 2006). Accordingly, a large fraction of clusters are expected to be elongated in the plane of the sky. On average, this will lead to an underestimation of the cluster mass when spherical symmetry is assumed (Rasia et al. 2012). Numerical simulations show that, for a sample of randomly oriented prolate clusters, their mass estimates are biased low by $\lesssim 5 \%$ on average when the masses are recovered from the projected mass distributions $\Sigma$ under the assumption of spherical symmetry (M14).

\subsubsection{Shear-Magnification Consistency}

Measuring the shear and magnification effects provides a consistency check of weak-lensing measurements, thereby allowing us to assess the robustness of our cluster mass estimates. Here we compare our mass estimates based on the $\boldsymbol{\Sigma}$ profiles recovered from the joint shear+magnification analysis with those obtained using the standard shear-only approach. Since background samples defined in different color regions (Section 4.4) suffer different degrees of (if any) contamination by cluster members, this comparison is sensitive to the presence of residual contamination of the background and to the shear calibration uncertainty. To do this, we adopt the Bayesian inference approach described in Section 5.3 and fit the NFW model to the tangential reducedshear profiles $\left\langle g_{+}\right\rangle$in the range $R \leqslant 2 \mathrm{Mpc} h^{-1}$.

In Figure 4, we show for our sample the ratio of cluster masses obtained using these two weak-lensing methods as a function of spherical radius. At each cluster radius, we compute the unweighted geometric mean and median of the shearonly to shear+magnification mass ratios. Note that we use geometric averaging, which satisfies $\langle Y / X\rangle=1 /\langle X / Y\rangle$ (see also Donahue et al. 2014). We see that the averaged mass ratio is consistent with unity within the errors but increases monotonically with cluster radius from $0.95 \pm 0.08$ at $r \simeq$ $200 \mathrm{kpc} h^{-1}$ to $1.08 \pm 0.09$ at $r \simeq 2 \mathrm{Mpc} h^{-1}$. This systematic radial trend can be explained by the $c-M$ degeneracy discussed in Section 5.4.1. On the basis of this comparison, we estimate the systematic uncertainty in the overall mass calibration to be of the order of $\pm 8 \%$. 


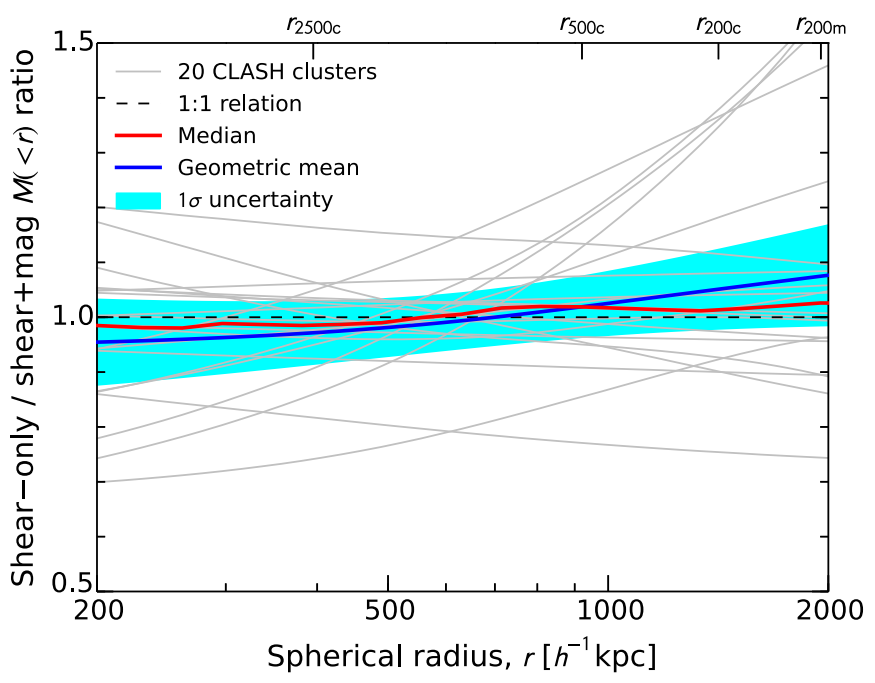

Figure 4. Ratio of cluster masses $M_{3 \mathrm{D}}(<r)$ obtained from NFW fits to the tangential reduced-shear profile (shear-only) and to the surface mass density profile reconstructed from the joint shear+magnification analysis (shear+mag). The results are shown for our sample of $20 \mathrm{CLASH}$ clusters (gray lines). The red line represents the median mass ratio as a function of spherical radius. The blue line and cyan-shaded area show the geometric-mean mass ratio and its $1 \sigma$ uncertainty, respectively. The dashed horizontal line marks the 1:1 relation.

(A color version of this figure is available in the online journal.)

\section{CLASH STACKED-LENSING ANALYSIS}

Stacking an ensemble of clusters helps average out the projection effects of cluster asphericity and substructure, as well as the cosmic noise from projected uncorrelated LSS, inherent in lensing measurements. The statistical precision can be greatly improved by stacking together a large number of clusters, especially on small angular scales (Okabe et al. 2010), allowing a tighter comparison of the averaged lensing profile with theoretical models.

Here our stacked-lensing analysis will focus on the CLASH X-ray-selected subsample of Postman et al. (2012), which comprises a population of high-mass X-ray regular clusters. The four high-magnification clusters are thus excluded from this part of the analysis.

In Section 6.1.1 we present a stacked tangential-distortion (shear-only) analysis of the $16 \mathrm{X}$-ray regular clusters and examine the form of their underlying halo mass profile using the ensemble-averaged $\left\langle\left\langle\boldsymbol{\Delta} \boldsymbol{\Sigma}_{+}\right\rangle\right\rangle$profile. In Section 6.2 we derive the ensemble-averaged total mass profile $\langle\langle\Sigma\rangle\rangle$ from our cluster mass-profile data set (Figure 3), for comparison with theoretical predictions taking into account both one- and two-halo term contributions.

\subsection{CLASH Stacked Shear-only Analysis}

\subsubsection{Stacking the Weak Shear Signal}

The azimuthally averaged tangential distortion is a measure of the radially modulated surface mass density and is insensitive to sheet-like mass overdensities, which resemble the projected two-halo term within a couple of virial radii (Oguri \& Hamana 2011). Hence, the stacked tangential-distortion signal around a large sample of clusters is a sensitive probe of the cluster-only (one-halo term) mass distribution.

In Figure 5 we show the stacked tangential-shear profile $\left\langle\left\langle\Delta \Sigma_{+}\right\rangle\right\rangle$derived for our sample where individual clusters and background galaxies are weighted by the shear-sensitivity kernel $\left(\operatorname{tr} \mathcal{W}_{+}\right.$in Section 3.4.1). The individual profiles are co-added in physical length units across the range $R=\left[R_{\min }, R_{\max }\right]=$

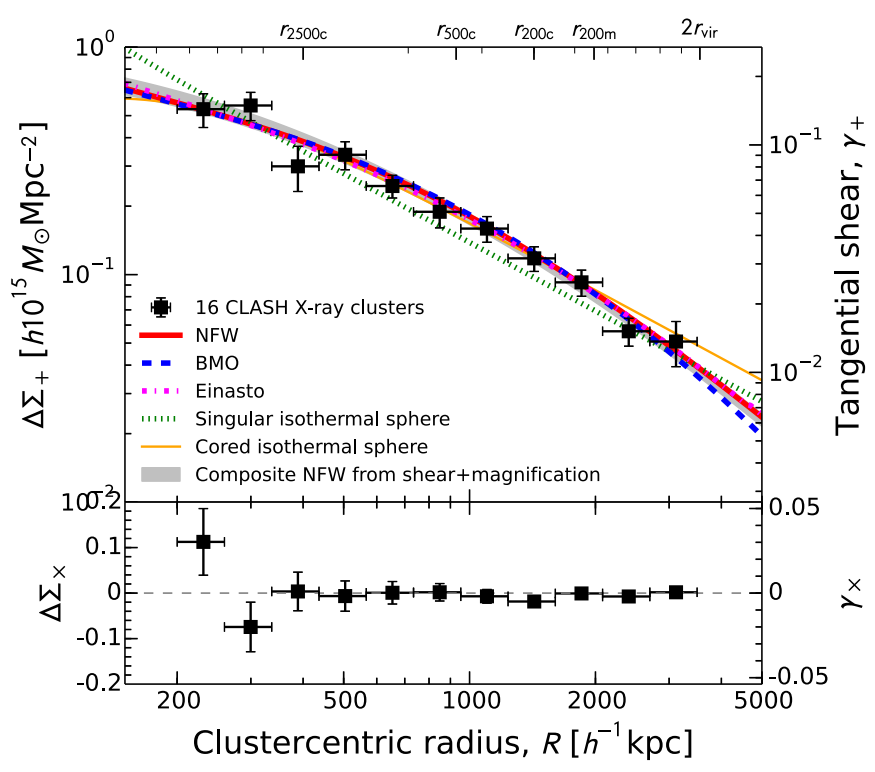

Figure 5. Average tangential-shear profile $\left\langle\left\langle\Delta \Sigma_{+}\right\rangle\right\rangle$(upper panel, black squares) obtained from stacking the X-ray-selected subsample of 16 clusters, shown in units of projected mass density. The thick-solid (red), dashed (blue), dotdashed (magenta), dotted (green), and thin-solid (orange) lines correspond to the best-fit NFW, truncated-NFW (Baltz et al. 2009, BMO), Einasto, SIS, and cored isothermal sphere profiles, respectively. The gray-shaded area shows the composite halo mass-profile prediction $(1 \sigma)$ derived from a weighted average of individual NFW profiles based on the joint shear+magnification analysis (Figure 3), in good agreement with the stacked-shear-only constraints (Figure 6). The lower panel shows the $45^{\circ}$ rotated $\times$ component $\left\langle\left\langle\Delta \Sigma_{\times}\right\rangle\right\rangle$, which is consistent with a null signal well within $2 \sigma$ at all radii, indicating the reliability of our distortion analysis. The right vertical axes represent the corresponding shear components, $\gamma_{+}=\left\langle\left\langle\Sigma_{\mathrm{c}}^{-1}\right\rangle\right\rangle\left\langle\left\langle\Delta \Sigma_{+}\right\rangle\right\rangle$and $\gamma_{\times}=\left\langle\left\langle\Sigma_{\mathrm{c}}^{-1}\right\rangle\right\rangle\left\langle\left\langle\Delta \Sigma_{\times}\right\rangle\right\rangle$, scaled to the mean depth of weak-lensing observations.

(A color version of this figure is available in the online journal.)

[200, 3500] kpc $h^{-1}$, in $11 \log$-spaced bins. Here, the radial limits $\left[R_{\min }, R_{\max }\right]$ of our stacking analysis represent approximately the respective median values of the radial boundaries $\left[\theta_{\min }, \theta_{\max }\right]$ covered by the data for our clusters at $0.19 \lesssim z_{1} \lesssim 0.69$. For individual clusters, we impose their respective radial cuts $\left[\theta_{\min }, \theta_{\max }\right]$ on the background samples, to be consistent with our individual cluster analysis. For our sample, we find a sensitivity-weighted average redshift of $\left\langle\left\langle z_{1}\right\rangle\right\rangle=0.345$, in close agreement with the median redshift of $\bar{z}_{1}=0.352$. The effective lensing sensitivity $\left\langle\left\langle\Sigma_{\mathrm{c}}^{-1}\right\rangle\right\rangle$ (Equation (22)) is $1 /\left\langle\left\langle\Sigma_{\mathrm{c}}^{-1}\right\rangle\right\rangle \simeq$ $3.88 \times 10^{15} h M_{\odot} \mathrm{Mpc}^{-2}$.

We detect the stacked-lensing signal at a total $\mathrm{S} / \mathrm{N}$ of $\simeq 25$ using the full covariance matrix $\mathcal{C}_{+}$(Equation (20)) to take into account intrinsic ellipticity and projected uncorrelated LSS noise, photo- $z$ uncertainties in the mean-depth calibration, and profile variations in individual clusters. The $45^{\circ}$ rotated $\times$ component $\left\langle\left\langle\boldsymbol{\Delta} \boldsymbol{\Sigma}_{\times}\right\rangle\right\rangle$is consistent with a null signal within $2 \sigma$ at all radii, with a total $\mathrm{S} / \mathrm{N}$ of $\simeq 2.8$, indicating that residual systematic errors are at least an order of magnitude smaller than the measured lensing signal.

\subsubsection{Modeling the Stacked Weak Shear Signal}

Here we quantify and characterize the ensemble-averaged mass distribution of our cluster sample using the stacked tangential-distortion signal. We examine the following five models for the halo mass density profile, $\rho(r)=$ $d M_{3 \mathrm{D}}(<r) / d r /\left(4 \pi r^{2}\right)$, each described by $N_{\mathrm{p}}$ parameters. 
Table 7

Best-fit Models for the Stacked Distortion Profile of the X-Ray-selected Subsample

\begin{tabular}{lcccc}
\hline \hline Model & $\begin{array}{c}M_{200 \mathrm{c}} \\
\left(10^{14} M_{\odot} h_{70}^{-1}\right)\end{array}$ & $c_{200 \mathrm{c}}$ & Structural Parameter & $\chi_{\min }^{2} / \mathrm{dof}^{\mathrm{a}}$ \\
\hline SIS & $10.4_{-0.6}^{+0.6}$ & $\ldots$ & $\ldots$ & 0.00 \\
Isothermal $\beta$ & $14.2_{-1.2}^{+1.2}$ & $\ldots$ & $r_{200 \mathrm{c}} / r_{c}=16.5_{-1.6}^{+2.4}$ & 0.33 \\
NFW & $13.4_{-0.9}^{+1.0}$ & $4.01_{-0.32}^{+0.35}$ & $\ldots$ & $0.3 / 9$ \\
NFW+pm & $13.1_{-1.1}^{+1.2}$ & $3.66_{-0.50}^{+0.68}$ & $M_{p}=(11 \pm 33) \times 10^{12} M_{\odot}$ & $6.8 / 9$ \\
BMO & $13.1_{-0.9}^{+0.9}$ & $3.73_{-0.31}^{+0.33}$ & $\ldots$ & $6.7 / 8$ \\
Einasto & $13.2_{-1.0}^{+1.0}$ & $3.73_{-0.52}^{+0.43}$ & $\alpha_{E}=0.191_{-0.068}^{+0.071}$ & 0.57 \\
\hline
\end{tabular}

Notes.

${ }^{\text {a }}$ Minimum $\chi^{2}$ per degrees of freedom (dof).

${ }^{\mathrm{b}}$ Probability to exceed (PTE) the given $\chi_{\min }^{2} /$ dof based on the standard $\chi^{2}$ probability distribution function.

1. Singular isothermal sphere (SIS) model with $N_{\mathrm{p}}=1$ :

$$
\rho_{\mathrm{SIS}}(r)=\frac{\sigma_{v}^{2}}{2 \pi G r^{2}},
$$

with $\sigma_{v}$ the one-dimensional isothermal velocity dispersion. 2. Isothermal- $\beta$ model with $N_{\mathrm{p}}=2$ (Hattori et al. 1999):

$$
\rho_{\text {iso }}(r)=\frac{M_{\mathrm{c}}}{2 \pi r_{\mathrm{c}}^{3}} \frac{3+\left(r / r_{\mathrm{c}}\right)^{2}}{\left[1+\left(r / r_{\mathrm{c}}\right)^{2}\right]^{2}},
$$

with $M_{\mathrm{c}}=M_{3 \mathrm{D}}\left(<r_{\mathrm{c}}\right)$ the total mass enclosed within the core radius $r_{\mathrm{c}}$. Note $\rho_{\text {iso }}(r) \propto r^{-2}$ at $r \gg r_{\mathrm{c}}$.

3. NFW model with $N_{\mathrm{p}}=2: \rho_{\mathrm{NFW}}(r)$ by Equation (25).

4. Baltz-Marshall-Oguri truncated-NFW model with $N_{\mathrm{p}}=2$ (Baltz et al. 2009, BMO):

$$
\rho_{\mathrm{BMO}}(r)=\frac{\rho_{\mathrm{s}}}{\left(r / r_{\mathrm{s}}\right)\left(1+r / r_{\mathrm{s}}\right)^{2}}\left[\frac{1}{1+\left(r / r_{\mathrm{t}}\right)^{2}}\right]^{2},
$$

with $r_{\mathrm{t}}=2.6 r_{\mathrm{vir}}\left(\sim 3 r_{200 \mathrm{c}}\right)$ the truncation radius, where the ratio of the truncation to virial radius, $\tau_{\mathrm{v}} \equiv r_{\mathrm{t}} / r_{\mathrm{vir}}$, is fixed to the typical value for cluster-sized halos in the $\Lambda \mathrm{CDM}$ cosmology (Oguri \& Hamana 2011).

5. Einasto model with $N_{\mathrm{p}}=3$ (Einasto 1965):

$$
\rho_{\mathrm{E}}(r)=\rho_{\mathrm{s}} \exp \left[-\frac{2}{\alpha_{\mathrm{E}}}\left(\frac{r}{r_{\mathrm{s}}}\right)^{\alpha_{\mathrm{E}}}\right],
$$

with $\alpha_{\mathrm{E}}$ the shape parameter describing the degree of curvature. An Einasto profile with $\alpha_{\mathrm{E}} \approx 0.18$ closely resembles the NFW profile over roughly two decades in radius (Ludlow et al. 2013). The logarithmic density gradient equals -2 at $r=r_{\mathrm{s}}$.

The NFW, BMO, and Einasto density profiles represent a family of phenomenological models for DM halos motivated by simulations and observations. The SIS profile with $\rho(r) \propto r^{-2}$ is often adopted as a lens model for its simplicity. The isothermal- $\beta$ model describes the total gravitating mass profile for isothermal gas with a $\beta$ density profile (Cavaliere \& Fusco-Femiano 1978). ${ }^{33}$

For the NFW, BMO, and Einasto models, we define the halo concentration parameter by $c_{200 \mathrm{c}}=r_{200 \mathrm{c}} / r_{\mathrm{s}}$. We specify the

\footnotetext{
33 For this model, $M_{\mathrm{c}}=\left(3 \beta k_{\mathrm{B}} T r_{\mathrm{c}}\right) /\left(2 G \mu m_{\mathrm{p}}\right)$, with $\beta$ the gas-density slope parameter, $T$ the gas temperature, $k_{\mathrm{B}}$ the Boltzmann constant, $\mu$ the mean molecular weight, and $m_{\mathrm{p}}$ the proton mass.
}

NFW and BMO models with $\left(M_{200 \mathrm{c}}, c_{200 \mathrm{c}}\right)$, the Einasto model with $\left(M_{200}, c_{200}, \alpha_{\mathrm{E}}\right)$, the isothermal- $\beta$ model with $\left(M_{200 \mathrm{c}}, r_{\mathrm{c}}\right)$, and the SIS model with $M_{200 c}$. For all cases, we can ignore the two-halo contribution to $\Delta \Sigma_{+}$, which is estimated to be $\gamma_{+} \lesssim 10^{-3}$ within our radial range $R \lesssim 2 r_{\text {vir }}$ and is an order of magnitude smaller than the observed lensing signal (see Figure 5).

We constrain the model parameters with our $\left\langle\left\langle\widehat{\Delta \Sigma_{+}}\right\rangle\right\rangle$profile and its full covariance matrix $\mathcal{C}_{+}$(Section 3.4.1). We use Equation (21) to make predictions for $\left\langle\left\langle\widehat{\Delta \Sigma_{+}}\right\rangle\right\rangle$. The $\chi^{2}$ minimization is performed using the MINUIT minimization package from the CERN program libraries. The best-fit parameters are reported in Table 7 , along with the reduced $\chi^{2}$ and corresponding probability-to-exceed (PTE) values. The NFW, $\mathrm{BMO}$, and Einasto models provide excellent fits to the data with PTE values of $0.66,0.58$, and 0.51 , respectively. The isothermal $\beta$ model yields a poorer but acceptable fit with a PTE of 0.33, while the SIS model gives an unacceptable fit of $\mathrm{PTE}=4.7 \times 10^{-3}$. The SIS model is disfavored at $4.3 \sigma$ significance from a likelihood-ratio test based on the differenced $\chi^{2}$ values $\Delta \chi^{2} \equiv \chi_{\text {SIS, min }}^{2}-\chi_{\mathrm{NFW} \text {, min }}^{2} \simeq 18.6$ relative to the $\mathrm{NFW}$ model. If the truncation radius of the BMO model is allowed to vary, we obtain $\tau_{v}=(2 \pm 106) \times 10^{2}(\mathrm{PTE}=0.56)$, which, however, makes the model essentially equivalent to the NFW profile.

In what follows, we will focus on our best models among those studied here, namely, the NFW, Einasto, and BMO density profiles. These models constrain $c_{200 \mathrm{c}}$ in the range $(1 \sigma)$ $3.2 \lesssim c_{200 \mathrm{c}} \lesssim 4.4\left(c_{200 \mathrm{c}}=4.01_{-0.32}^{+0.35}\right.$ for NFW), for our 16 $\mathrm{X}$-ray-selected clusters with $M_{200 \mathrm{c}}=(1.3 \pm 0.1) \times 10^{15} M_{\odot} h_{70}^{-1}$. For the NFW model, we find $r_{\mathrm{s}} \simeq 360 \mathrm{kpc} h^{-1}$, so that our data cover the range $0.6 \lesssim R / r_{\mathrm{s}} \lesssim 10$.

\subsubsection{Uncertainty in Halo Profile Shape}

To examine the impact of the uncertainty in profile shape, we compare in Figure 6 the spherical mass profiles $M_{\Delta_{c}}$ for the bestfit NFW, BMO, and Einasto models at several overdensities. These profiles are nearly identical at $\Delta_{\mathrm{c}} \geqslant 200$. At $\Delta_{\mathrm{c}}=100$, the BMO-to-NFW and Einasto-to-NFW mass ratios are $0.95 \pm 0.10$ and $1.03 \pm 0.14$, respectively, both consistent with unity.

Also shown in Figure 6 is the composite profile $\left\langle\left\langle M_{\Delta}\right\rangle\right\rangle$ for our sample constructed from a weighted average of the individual mass estimates (Section 5.3) constrained by the combined shear+magnification measurements (Figures 2 and 3). 


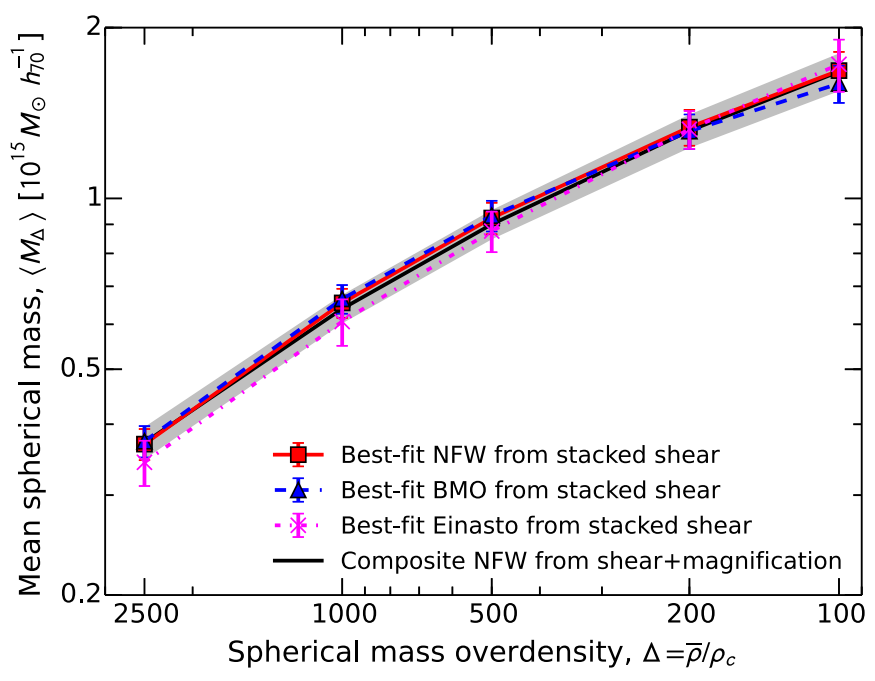

Figure 6. Mean cumulative mass profiles $M_{\Delta}$ of the ensemble of $16 \mathrm{CLASH}$ $\mathrm{X}$-ray-selected clusters derived from different weak-lensing analysis methods, shown at five characteristic values of the spherical mass overdensity, $\Delta \equiv$ $\rho(<r) / \rho_{\mathrm{c}}$. The squares, triangles, and crosses with error bars, respectively, are the best-fit NFW, truncated-NFW (Baltz et al. 2009, BMO), and Einasto profiles with $1 \sigma$ uncertainty derived from the stacked shear-only analysis (Figure 5), demonstrating the effects of the uncertainty in halo profile shape. The black solid line and gray-shaded area show the mean and $1 \sigma$ uncertainty of the composite profile $\left\langle\left\langle M_{\Delta}\right\rangle\right\rangle$ from a weighted average of NFW fits to individual mass profiles $\Sigma(R)$ reconstructed from the shear+magnification constraints (Figure 2).

(A color version of this figure is available in the online journal.)

Specifically, $\left\langle\left\langle M_{\Delta}\right\rangle\right\rangle$ is defined by

$$
\left\langle\left\langle M_{\Delta}\right\rangle\right\rangle=\frac{\sum_{n=1}^{N_{\mathrm{cl}}} \operatorname{tr}\left(\mathcal{W}_{+, n}\right) M_{\Delta, n}}{\sum_{n=1}^{N_{\mathrm{cl}}} \operatorname{tr}\left(\mathcal{W}_{+, n}\right)}
$$

$\left(N_{\mathrm{cl}}=16\right)$, using the shear-sensitivity kernel $\operatorname{tr}\left(\mathcal{W}_{+}\right)$. At $\Delta_{\mathrm{c}}=200, \quad\left\langle\left\langle M_{200 \mathrm{c}}\right\rangle\right\rangle=(1.32 \pm 0.08) \times 10^{15} M_{\odot} h_{70}^{-1}$, in excellent agreement with the best-fit NFW halo mass, $M_{200 \mathrm{c}}=$ $1.34_{-0.09}^{+0.10} \times 10^{15} M_{\odot} h_{70}^{-1}$, from the stacked-shear-only analysis (Table 7). These comparisons show that our results are robust against different ensemble-averaging techniques and data combinations, and that the uncertainty in profile shape does not significantly affect our cluster mass measurement within the virial radius. Similarly, the composite NFW prediction for $\left\langle\left\langle\Delta \Sigma_{+}\right\rangle\right\rangle$ is shown in Figure 5, demonstrating consistency between the shear and magnification measurements.

On the other hand, the effects of baryonic physics can in principle affect the mass density profile in the central highdensity region of the cluster. Here we turn to assess the possible impact of adiabatic contraction on the total measured mass profile (Gnedin et al. 2004; Okabe et al. 2013) by introducing a central point mass into our modeling. The results for the combined NFW and point-mass (NFW+pm) model are listed in Table 7. For the central point-mass component, we obtain $M_{\mathrm{p}}=(11 \pm 33) \times 10^{12} M_{\odot} h_{70}^{-1}$, or a $1 \sigma$ upper limit of $M_{\mathrm{p}}<44 \times 10^{12} M_{\odot} h_{70}^{-1}$ within $R_{\min }=200 \mathrm{kpc} h^{-1}$. Including this additional degree of freedom to account for the central unresolved mass component does not significantly change the best-fit mass and concentration parameters for our data (Table 7); however, it reduces the lower bound on $c_{200}$, allowing for somewhat lower concentrations $\left(c_{200 \mathrm{c}} \gtrsim 3.2\right.$ at $\left.1 \sigma\right)$.

\subsection{CLASH Stacked Mass Profile Analysis}

We show in Figure 7 the averaged total mass profile $\langle\langle\boldsymbol{\Sigma}\rangle\rangle$,

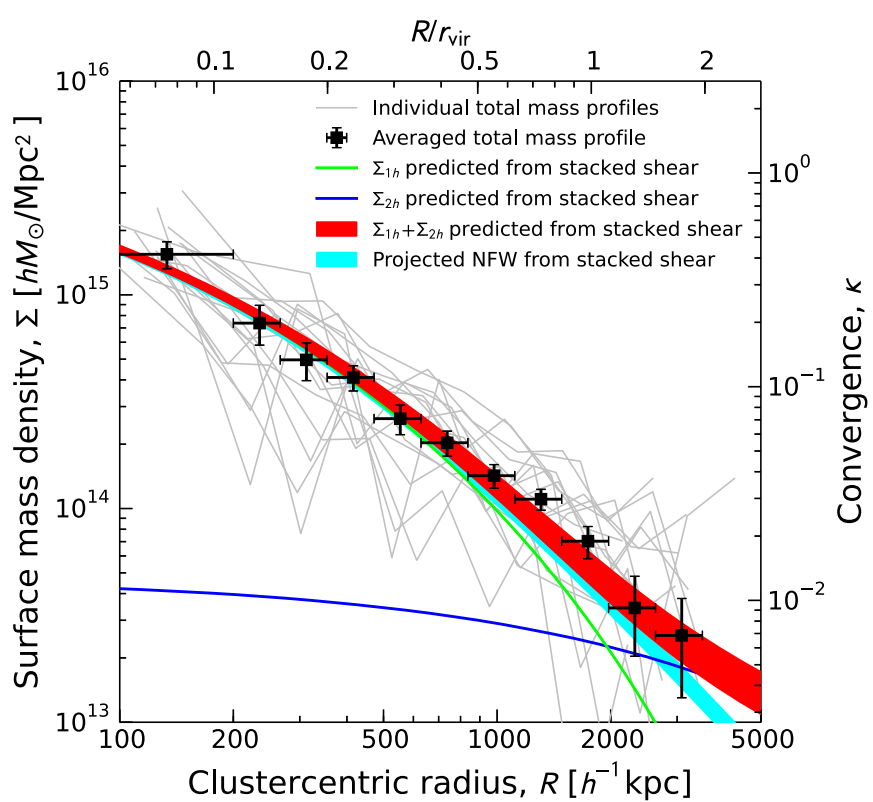

Figure 7. Averaged total projected mass profile (black squares) of the X-rayselected subsample, which is obtained by stacking individual mass profiles (gray lines; Figure 3) derived from our shear+magnification data (Figure 2). The green and blue solid lines, respectively, are the one-halo $\left(\Sigma_{1 \mathrm{~h}}\right)$ and two-halo $\left(\Sigma_{2 \mathrm{~h}}\right)$ contributions predicted by our best-fit model from the stacked-shear-only analysis (Figure 5). The red-shaded area shows the sum of the predicted $\Sigma_{1 \mathrm{~h}}$ and $\Sigma_{2 \mathrm{~h}}$ components $(68 \% \mathrm{CL})$, in agreement with the observed total mass profile based on the combination of shear and magnification. The projected NFW model (cyan-shaded area, 68\% CL) based on the stacked shear-only constraints slightly underpredicts the observed mass profile at $R \gtrsim r_{\text {vir }}$. The scale on the right vertical axis indicates the corresponding lensing convergence, $\kappa=\left\langle\left\langle\Sigma_{\mathrm{c}}^{-1}\right\rangle\right\rangle\langle\langle\Sigma\rangle\rangle$, scaled to the mean depth of weak-lensing observations.

(A color version of this figure is available in the online journal.)

which is obtained by stacking the individual cluster profiles $\boldsymbol{\Sigma}$ derived from the shear+magnification constraints. We take $R_{\min }=200 \mathrm{kpc} h^{-1}$ and $R_{\max }=3500 \mathrm{kpc} h^{-1}$ to be consistent with the stacked-shear-only analysis (Section 6.1.1). The effective lensing sensitivity for this analysis is $1 /\left\langle\left\langle\Sigma_{\mathrm{c}}^{-1}\right\rangle\right\rangle \simeq$ $3.98 \times 10^{15} h M_{\odot} \mathrm{Mpc}^{-2}$, which is obtained using $\operatorname{tr}\left(\mathcal{W}_{n}\right)$ as statistical weights (Section 3.4.2). The averaged mass profile $\langle\langle\Sigma\rangle\rangle$ is detected at a total $\mathrm{S} / \mathrm{N}$ of $\simeq 21$, which is $\simeq 16 \%$ lower than that of the stacked distortion signal $\left\langle\left\langle\Delta \Sigma_{+}\right\rangle\right\rangle$(Section 6.1.1). This reduction in $\mathrm{S} / \mathrm{N}$ is due primarily to significant cluster-to-cluster variations between the individual total $\Sigma$ profiles. We have also checked that our stacking results are insensitive to whether or not individual cluster profiles are scaled to their $r_{\text {vir }}$ prior to averaging albeit with some variance in the outermost radial bin.

Here we examine the consistency of our ensemble-averaged shear-only and shear+magnification results in the context of the standard $\Lambda \mathrm{CDM}$ model. To do this, we employ the halomodel prescriptions of Oguri \& Takada (2011) and Oguri \& Hamana (2011) for computing the two-halo contribution $\Sigma_{2 h}$ to the total projected matter distribution $\Sigma=\Sigma_{1 \mathrm{~h}}+\Sigma_{2 \mathrm{~h}}$ expected from the stacked shear constraints on the one-halo component $\Sigma_{\mathrm{h}}$. For an ensemble of clusters with mass $M$ and redshift $z$, the $\Sigma_{2 \mathrm{~h}}$ component is computed by projecting the two-halo term $\rho_{2 \mathrm{~h}}(r)=\bar{\rho} b_{\mathrm{h}}(M) \xi_{\text {lin }}(r)$ along the line of sight (see Section 2.2 of Oguri \& Hamana 2011), with $\bar{\rho}$ the mean background density of the universe, $b_{\mathrm{h}}(M)$ the linear halo bias, ${ }^{34}$ and $\xi_{\operatorname{lin}}(r)$ the

\footnotetext{
34 The Tinker et al. (2010) model is given as a function of the interior overdensity $\Delta_{m}$ defined with respect to the mean background density $\bar{\rho}(z)$ of the universe. In the present study we take $\Delta_{\mathrm{c}}=200$, corresponding to $\Delta_{\mathrm{m}} \sim 420$ at $z=0.35$ for our adopted cosmology.
} 
linear matter correlation function, all evaluated at $z=\left\langle\left\langle z_{1}\right\rangle\right\rangle$ in the WMAP seven-year cosmology (Section 3.3.2). The two-halo term is proportional to the product $b_{\mathrm{h}}(M) \sigma_{8}^{2}$, where $\sigma_{8}^{2}$ is the rms amplitude of linear mass fluctuations in a sphere of comoving radius $r=8 \mathrm{Mpc} h^{-1}$.

To estimate the halo bias $b_{\mathrm{h}}(M)$, we adopt the model of Tinker et al. (2010), which is well calibrated using a large set of $N$-body simulations. With this, we find $b_{\mathrm{h}}=9.0 \pm 0.5 \pm 2$ for our best-fit mass model from the stacked-shear-only analysis, where the former error reflects the uncertainty in the mass estimate and the latter represents the model uncertainty of $\sim 20 \%$ in the fitting function of Tinker et al. (2010) in the high-peak, high-bias regime. We use our best-fit BMO model in Table 7 to represent the $\Sigma_{1 \mathrm{~h}}$ component.

In Figure 7, we compare the observed $\langle\langle\Sigma\rangle\rangle$ profile with the resulting shear-based predictions for the one-halo $\left(\Sigma_{1 \mathrm{~h}}\right)$, twohalo $\left(\Sigma_{2 \mathrm{~h}}\right)$, and total $\left(\Sigma_{1 \mathrm{~h}}+\Sigma_{2 \mathrm{~h}}\right)$ components. The two-halo component $\Sigma_{2 \mathrm{~h}}$ slowly decreases from $\kappa_{2 \mathrm{~h}} \equiv\left\langle\left\langle\Sigma_{\mathrm{c}}^{-1}\right\rangle\right\rangle \Sigma_{2 \mathrm{~h}} \sim 10^{-2}$ in the central region to $\kappa_{2 \mathrm{~h}} \sim 4 \times 10^{-3}$ at $R \sim 2 r_{\text {vir }}$ and thus mimics a massive uniform sheet around the clusters. Figure 7 shows that the halo-model predictions for the total signal agree well with our results, indicating good consistency between our shear and magnification measurements in the context of the standard $\Lambda C D M$ model. On the other hand, the projected NFW model based on the stacked shear-only constraints slightly underpredicts the observed mass profile at $R \gtrsim r_{\text {vir }}$, where the two-halo contribution is important.

\section{DISCUSSION}

\subsection{Mass Comparisons}

In this subsection, we compare our mass estimates for the CLASH sample (Table 6) with those from previous studies. More detailed statistical comparisons of cluster mass determinations for the CLASH sample by different lensing, Xray, SZE, and dynamical methods will be presented in our forthcoming papers.

\subsubsection{Previous CLASH Lensing Results}

Now we compare our estimates of $M_{\text {vir }}$ obtained by our uniform analysis with those from our previous work on individual CLASH clusters, namely, A2261 (Coe et al. 2012), MACS J1206.2-0847 (Umetsu et al. 2012), and MACS J0717.5+3745 (Medezinski et al. 2013). The major differences between this and previous weak-lensing analyses are summarized as follows.

1. We have applied a shear calibration correction factor of $1 / 0.95 \simeq 1.05$ (Section 4.3), which was not included in our previous studies of A2261 and MACS J1206.2-0847.

2. The nonlinear correction given by Equation (7) was not included in our previous work.

3. All mean depth estimates have been uniformly performed here using a self-consistent photo- $z$ method as described in Section 4.4

4. A Bayesian inference approach has been used here to measure cluster masses at several overdensities, by limiting the fitting range to $R \leqslant 2 \mathrm{Mpc} h^{-1}$. The $\chi^{2}$ minimization was performed in our previous work to derive the bestfit virial mass and concentration parameters for the full radial range.

Besides, in earlier papers, we assumed a slightly different cosmology with $\left(\Omega_{\mathrm{m}}, \Omega_{\Lambda}\right)=(0.3,0.7)$. As a result, these changes lead to a typical increase of $\sim 10 \%$ in our $M_{\text {vir }}$ estimates relative to our previous work. This increase is primarily due to the inclusion of the shear calibration correction, which translates into a typical increase of $\sim 8 \%$ in $M_{\text {vir }}$. On the other hand, the present analysis takes into account systematic uncertainties $C^{\text {sys }}$ (Equation (16)) due primarily to the residual mass-sheet degeneracy, providing more conservative error estimates for clusters with poorer magnification constraints.

The following is a detailed comparison of each cluster.

A2261. Coe et al. (2012) obtained $M_{\text {vir }}=2.21_{-0.23}^{+0.25} \times$ $10^{15} M_{\odot} h_{70}^{-1}$ from an NFW fit to the mass profile from their shear+magnification data (Constraints (8) of their Table 4), with an estimated correction of $\Delta M_{\mathrm{vir}} \simeq 0.15 \times 10^{15} M_{\odot} h_{70}^{-1}$ for projection effects due to LSS as specifically observed behind A2261. This is compared to $M_{\text {vir }}=(2.58 \pm 0.54) \times 10^{15} M_{\odot} h_{70}^{-1}$ in this work, corresponding to an increase of $17 \%$ in the best-fit $M_{\text {vir }}$, in which the revised estimates of mean depth result in a $\sim 5 \%$ increase in mass. The large increase in the uncertainty of $M_{\mathrm{vir}}$ is primarily caused by the inclusion of $C^{\text {sys }}$ (Equation (16)) due to the residual mass-sheet uncertainty, which dominates the error budget for the four outermost $\Sigma$ bins of A2261. A large contribution from $C^{\text {sys }}$ is generally expected for low- $z$, highmass clusters owing to their large angular extent on the sky.

MACS J1206.2-0847. Umetsu et al. (2012) found $M_{\mathrm{vir}}=$ $1.64_{-0.40}^{+0.49} \times 10^{15} M_{\odot} h_{70}^{-1}$ based on their shear+magnification measurements (Method (2) of their Table 5), compared to our estimate of $M_{\mathrm{vir}}=(1.87 \pm 0.46) \times 10^{15} M_{\odot} h_{70}^{-1}$, which represents an increase of $14 \%$. Their primary NFW model from full-lensing constraints (their Method (7)), including a correction for the surrounding LSS, yields $M_{500 \mathrm{c}}=(1.01 \pm$ $0.15) \times 10^{15} M_{\odot} h_{70}^{-1}$ (see also Rozo et al. 2014), which agrees well with our estimate of $M_{500 \mathrm{c}}=(1.06 \pm 0.21) \times 10^{15} M_{\odot} h_{70}^{-1}$.

MACS J0717.5+3745. The cluster was recently studied by Medezinski et al. (2013), who derived $M_{\text {vir }}=3.19_{-0.54}^{+0.63} \times$ $10^{15} M_{\odot} h_{70}^{-1}$ based on their two-dimensional shear and azimuthally averaged magnification-bias measurements (their Table 5), ${ }^{35}$ assuming a composite model of an NFW halo and a constant component, where the latter accounts for the surrounding LSS. Their estimated $M_{\mathrm{vir}}$ is $11 \%$ lower than our estimate of $M_{\text {vir }}=(3.53 \pm 0.60) \times 10^{15} M_{\odot} h_{70}^{-1}$, as the correlated LSS contributions are partly absorbed into their mass-sheet parameter.

Finally, we compare our mass estimates of RX J2248.7-4431 with the results from a weak-shear analysis of Gruen et al. (2013) based on ESO/WFI data. For this cluster we use the same co-added images created by Gruen et al. (2013) but adopt substantially different approaches in our analysis (Section 4.2). They derived mass estimates of this cluster at several overdensities, $M_{200 \mathrm{~m}}=33.1_{-6.8}^{+9.6} \times 10^{15} M_{\odot} h_{70}^{-1}$, $M_{101 \mathrm{c}}=32.2_{-6.6}^{+9.3} \times 10^{15} M_{\odot} h_{70}^{-1}\left(\approx M_{100 \mathrm{c}}\right), M_{200 \mathrm{c}}=22.8_{-4.7}^{+6.6} \times$ $10^{15} M_{\odot} h_{70}^{-1}$, and $M_{500 \mathrm{c}}=12.7_{-2.6}^{+3.7} \times 10^{15} M_{\odot} h_{70}^{-1}$, which are all consistent within errors with ours (Table 6).

\subsubsection{The Weighing the Giants Project}

The majority of the CLASH clusters were targeted as part of the $\mathrm{WtG}$ project. Recently, the WtG collaboration published results of their weak-lensing shear mass measurements of 51 X-ray-luminous clusters at $0.15 \lesssim z \lesssim 0.7$ based on

\footnotetext{
35 The 5\% residual correction for shear calibration was included in the analysis by Medezinski et al. (2013).
} 


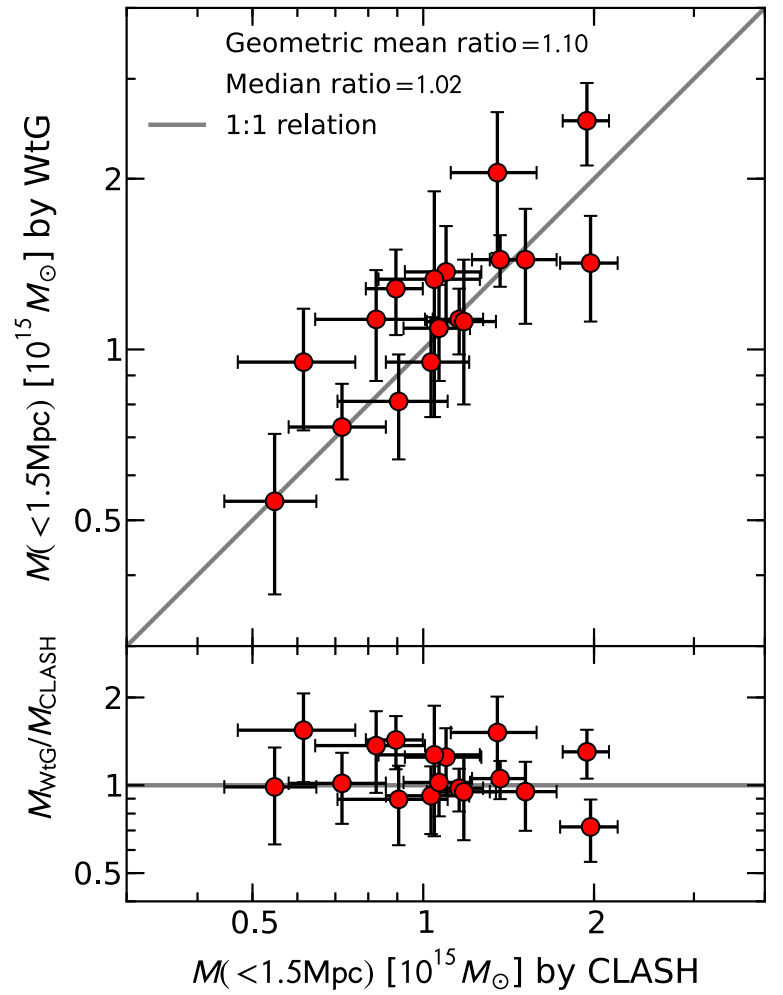

Figure 8. Comparison of Subaru shear-only mass estimates $\left(M_{\mathrm{WtG}}\right)$ from Applegate et al. (2014) to our Subaru shear+magnification results ( $\left.M_{\mathrm{CLASH}}\right)$ for 17 clusters in common between the two studies (upper panel, circles with error bars). For this comparison we measure the mass within a sphere of $r=1.5 \mathrm{Mpc} h_{70}^{-1}$ using the same cosmology $\left(h, \Omega_{\mathrm{m}}, \Omega_{\Lambda}\right)=(0.7,0.3,0.7)$ adopted by Applegate et al. (2014). The solid line shows the one-to-one relation. The lower panel shows $M_{\mathrm{WtG}} / M_{\mathrm{CLASH}}$ of individual clusters against $M_{\mathrm{CLASH}}$. (A color version of this figure is available in the online journal.)

deep multi-color Subaru/Suprime-Cam and CFHT/MegaPrime optical imaging (von der Linden et al. 2014; Kelly et al. 2014; Applegate et al. 2014).

Figure 8 shows a comparison of Subaru shear-based mass estimates $\left(M_{\mathrm{WtG}}\right)$ from Applegate et al. (2014) to our Subaru weak-lensing results. There are 17 clusters in common between the two studies, including $14 \mathrm{X}$-ray-selected and 3 highmagnification CLASH clusters. To make this comparison, we measure the mass $\left(M_{\mathrm{CLASH}}\right)$ within a fixed physical radius of $r=1.5 \mathrm{Mpc} h_{70}^{-1}$ assuming the spherical NFW model and using the same cosmology $\left(h, \Omega_{\mathrm{m}}, \Omega_{\Lambda}\right)=(0.7,0.3,0.7)$ adopted by Applegate et al. (2014). Applegate et al. (2014) derived cluster masses from NFW fits to their tangential reducedshear data over the radial range $R=0.75-3 \mathrm{Mpc} h_{70}^{-1}$, whereas our mass measurements are based on the lensing convergence $(\kappa)$ data over the range $R \leqslant 2 \mathrm{Mpc} h^{-1} \simeq$ $2.9 \mathrm{Mpc} h_{70}^{-1}$, constrained by the combined shear+magnification measurements. For this comparison, we use their results based on the photo- $z$ posterior probability distributions of individual galaxies where available; otherwise, we use those from their color-cut method. For these 17 clusters, we find a median $M_{\mathrm{WtG}} / M_{\mathrm{CLASH}}$ ratio of 1.02 and an unweighted geometric mean (Section 5.4.3) of $\left\langle M_{\mathrm{WtG}} / M_{\mathrm{CLASH}}\right\rangle=1.10 \pm 0.09$. For the error estimation, we have neglected the correlation between the two axes due to overlap of source galaxies used to measure the shear because our analysis includes independent constraints from the magnification effects on red galaxy counts. Considering the systematic uncertainty of $\pm 8 \%$ in our overall mass calibration

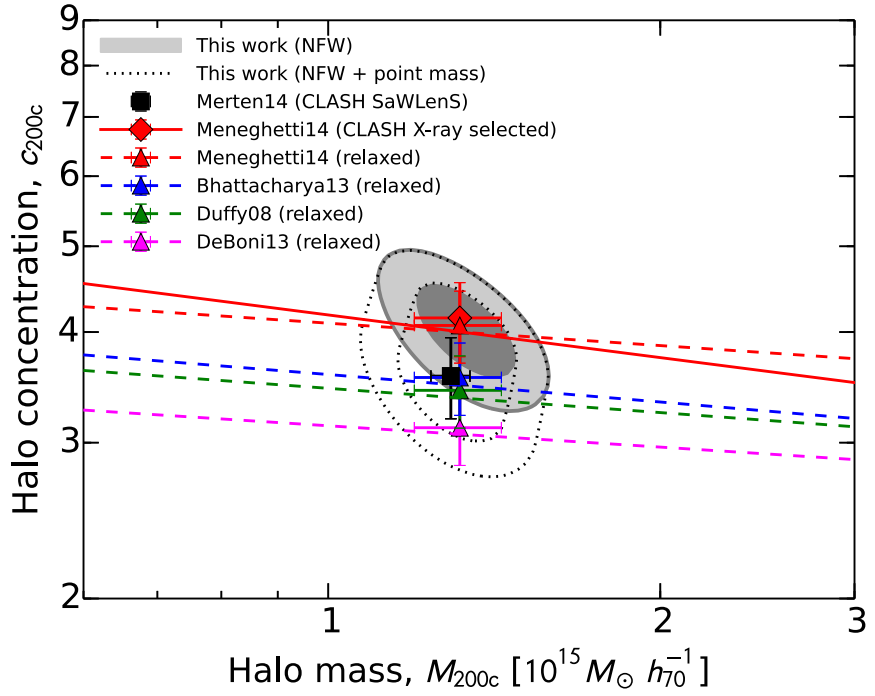

Figure 9. Stacked weak-shear constraints on the mass and concentration of 16 CLASH X-ray-selected clusters at $\left\langle\left\langle z_{1}\right\rangle\right\rangle \simeq 0.35$. The gray contours show the $68.3 \%$ and $95.4 \%$ confidence levels in the $c-M$ plane for the spherical NFW fit. Similarly, the black-dashed contours show the model fit including a central point-mass component, accounting for the possible impact of adiabatic contraction. The triangles with error bars show the predictions $\left\langle\left\langle c_{200 c}\right\rangle\right\rangle$ using the $c_{200 \mathrm{c}}\left(M_{200 \mathrm{c}}, z\right)$ relations for relaxed halos in the literature. The diamond with error bars represents the prediction for CLASH-like X-ray regular halos (Meneghetti et al. 2014). These predictions are obtained from the respective weighted averages of predicted $c$ values for the observed $\left(M_{200 c}, z\right)$ distribution of our sample, where the $M_{200 c}$ masses are based on the shear+magnification constraints. For each model, the stacked model prediction is consistent with the corresponding $c-M$ relation evaluated at $z=\left\langle\left\langle z_{1}\right\rangle\right\rangle$ (solid: X-ray; dashed: relaxed). The square represents the results from a strong- and weak-lensing (SAWLENS) analysis of 19 CLASH X-ray-selected clusters (Merten et al. 2014). (A color version of this figure is available in the online journal.)

(Section 5.4.4), we find no significant offset between our and $\mathrm{WtG}$ mass measurements.

\subsubsection{Rozo et al. (2014)}

Rozo et al. (2014) performed a self-consistent calibration of cluster scaling relations between X-ray, SZE, and optical observables. They used their calibrated mass-X-ray luminosity relation to predict masses $\left(M_{\mathrm{R} 14}\right)$ within the overdensity radius $r_{500 \mathrm{c}}$ for the CLASH clusters at $z \leqslant 0.4$ and MACS J1206.2-0847 at $z=0.44$. For each cluster we calculate the mass ( $\left.M_{\mathrm{CLASH}}\right)$ within the $r_{500 \mathrm{c}}$ of Rozo et al. (2014) assuming the spherical NFW model.

For 13 clusters in common with Rozo et al. (2014), we obtain a median mass ratio of 1.12 and an error-weighted geometric mean of $\left\langle M_{\mathrm{R} 14} / M_{\mathrm{CLASH}}\right\rangle=1.13 \pm 0.10$, corresponding to a mass offset of $\Delta \ln M \equiv\left\langle\ln M_{\mathrm{R} 14}\right\rangle-\left\langle\ln M_{\mathrm{CLASH}}\right\rangle=0.12 \pm 0.09$. This offset is not significant compared to the overall calibration uncertainty of $\pm 8 \%$ (Section 5.4.4). Excluding two obvious outliers with $M_{\mathrm{R} 14} / M_{\mathrm{CLASH}} \sim 2$ (RX J 2129.7+0005 and RX J 1532.9+3021), we find a median mass ratio of 1.11 and a weighted geometric mean ratio of $1.03 \pm 0.10$, which corresponds to a mass offset of $\Delta \ln M=0.03 \pm 0.09$.

\subsection{Cluster c-M Relation}

Stacking the tangential distortion signal from a large sample of clusters can provide useful integrated constraints on the halo concentration-mass $(c-M)$ relation. In Figure 9, we summarize our stacked weak-shear constraints in the $c-M$ plane for our 16 CLASH X-ray-selected clusters. 
Table 8

Comparison with Numerical Simulations

\begin{tabular}{|c|c|c|c|c|c|c|}
\hline \multirow[t]{2}{*}{ Model } & \multicolumn{2}{|c|}{ Relaxed Sample } & \multicolumn{2}{|c|}{ Full Sample } & \multicolumn{2}{|c|}{ X-Ray Regular Sample } \\
\hline & $\left\langle\left\langle c_{200 c}\right\rangle\right\rangle$ & $c^{(\mathrm{obs})} / c^{(\mathrm{sim})}$ & $\left\langle\left\langle c_{200 c}\right\rangle\right\rangle$ & $c^{(\mathrm{obs})} / c^{(\mathrm{sim})}$ & $\left\langle\left\langle c_{200 c}\right\rangle\right\rangle$ & $c^{(\mathrm{obs})} / c^{(\mathrm{sim})}$ \\
\hline Duffy et al. (2008) & $3.44 \pm 0.32$ & $1.17 \pm 0.15$ & $3.02 \pm 0.28$ & $1.33 \pm 0.17$ & $\cdots$ & $\cdots$ \\
\hline Bhattacharya et al. (2013) & $3.55 \pm 0.33$ & $1.13 \pm 0.14$ & $3.43 \pm 0.32$ & $1.17 \pm 0.15$ & $\ldots$ & $\ldots$ \\
\hline De Boni et al. (2013) & $3.12 \pm 0.29$ & $1.29 \pm 0.16$ & $2.75 \pm 0.26$ & $1.46 \pm 0.18$ & $\cdots$ & $\cdots$ \\
\hline Meneghetti et al. (2014) & $4.07 \pm 0.38$ & $0.99 \pm 0.12$ & $3.77 \pm 0.35$ & $1.06 \pm 0.13$ & $4.15 \pm 0.40$ & $0.97 \pm 0.12$ \\
\hline
\end{tabular}

Notes. Predicted concentration $\left\langle\left\langle c_{200 \mathrm{c}}\right\rangle\right\rangle$ and observed-to-predicted ratio $c^{(\mathrm{obs})} / c^{(\mathrm{sim})}$ for the 16 CLASH X-ray-selected clusters. The best-fit NFW model is used for a baseline comparison with the numerical simulations. Here the theoretical predictions from Duffy et al. (2008), Bhattacharya et al. (2013), and De Boni et al. (2013) are based on DM-only simulations, and those from Meneghetti et al. (2014) are based on nonradiative simulations of DM and baryons.

\subsubsection{Comparison with $\Lambda$ CDM Predictions}

We compare our results with $\Lambda \mathrm{CDM}$ predictions from numerical simulations. Specifically, we examine the $c-M$ relations obtained by Duffy et al. (2008), Bhattacharya et al. (2013), De Boni et al. (2013), and M14 using the standard NFW model. Hence, we use our NFW results for a baseline comparison with the numerical simulations.

To make a quantitative comparison between theory and observations, we calculate, for a given $c(M, z)$ relation, the sensitivity-weighted average $\left\langle\left\langle c_{200 c}\right\rangle\right\rangle$ of concentrations for the observed mass and redshift distribution of our cluster sample, $\left\{M_{200 \mathrm{c}, n}\right\}_{n=1}^{N_{\mathrm{cl}}}$ (Table 6) and $\left\{z_{1, n}\right\}_{n=1}^{N_{\mathrm{cl}}}$ (Table 1), by

$$
\left\langle\left\langle c_{200 \mathrm{c}}\right\rangle=\frac{\sum_{n=1}^{N_{\mathrm{cl}}} \operatorname{tr}\left(\mathcal{W}_{+, n}\right) c_{200 \mathrm{c}}\left(M_{200 \mathrm{c}, n}, z_{1, n}\right)}{\sum_{n=1}^{N_{\mathrm{cl}}} \operatorname{tr}\left(\mathcal{W}_{+, n}\right)},\right.
$$

which is marked at the average cluster mass $\left\langle\left\langle M_{200 \mathrm{c}}\right\rangle\right\rangle$ (Section 6.1.2) in Figure 9. Note that the masses $\left\{M_{200 \mathrm{c}, n}\right\}_{n=1}^{N_{\mathrm{cl}}}$ are constrained by the combined shear+magnification data. We use Monte Carlo simulations to estimate the total uncertainty in $\left\langle\left\langle c_{200 c}\right\rangle\right\rangle$, taking into account both the observational uncertainties in the cluster mass measurements (Section 5.3) and the intrinsic scatter in the concentration. Here we assume a Gaussian intrinsic scatter of dispersion $\sigma_{c}=0.33 c_{200 \mathrm{c}}$ as found by Bhattacharya et al. (2013).

The resulting predictions are summarized in Table 8 and shown in Figure 9. Overall, the predicted concentrations $\left\langle\left\langle c_{200 \mathrm{c}}\right\rangle\right\rangle$ for relaxed halos overlap well with our range of allowed $c$ values, $3.2 \lesssim c_{200 \mathrm{c}} \lesssim 4.4$ at $1 \sigma(\mathrm{NFW}$ and NFW+pm). Our NFW results are in excellent agreement with the predicted concentration from nonradiative hydrodynamical $N$-body simulations in a $W M A P$ seven-year cosmology (M14, $\left.\sigma_{8}=0.82\right)$. Our measurements are slightly higher than, but consistent with, the DM-only predictions of Bhattacharya et al. $\left(2013, \sigma_{8}=0.8\right)$ and Duffy et al. $\left(2008, \sigma_{8}=0.796\right)$. We find a discrepancy of about $1.8 \sigma$ between our NFW results and the DM-only prediction of De Boni et al. (2013, $\left.\sigma_{8}=0.776\right)$. These differences can be partly explained by different cosmological settings, such as the adopted values of $\sigma_{8}$, as discussed in detail by M14. Alternatively, this discrepancy can be reconciled if the NFW+pm model (Figure 9) is adopted to account for the possible impact of unresolved adiabatic contraction (Section 6.1.2).

Using the $c-M$ relations derived for the full population of halos (including both relaxed and unrelaxed halos), we find that the predicted concentrations are 4\%-9\% lower than those for the relaxed halos (Table 8). The full-sample predictions of the Bhattacharya et al. (2013) and M14 models are consistent with our results within $\sim 1 \sigma$.
Finally, we examine the M14 predictions for a sample of CLASH-like X-ray-regular halos, including the effects of the CLASH X-ray selection function and possible biases due to projection effects. M14 characterized a sample of halos that reproduces the observed distribution of X-ray regularity characteristics in the CLASH X-ray-selected subsample. They found that a large fraction of this sample $(\sim 70 \%)$ is composed of relaxed halos, but it also contains a non-negligible fraction of unrelaxed halos. Matching their simulations to the individual CLASH clusters based on the X-ray morphology, M14 predict that the NFW concentrations recovered from the lensing analysis of the CLASH X-ray-selected clusters are in the range $3 \lesssim$ $c_{200 \mathrm{c}} \lesssim 6$. We find this model provides an excellent match to the observed concentration (Table 8).

\subsubsection{Comparison with the CLASH SAWLENS Analysis}

For a massive cluster acting as a supercritical lens, the strong- and weak-lensing regimes contribute similar logarithmic radial coverage (Umetsu et al. 2011b). Hence, adding stronglensing information to weak-lensing allows us to provide tighter constraints on the inner density profile. Merten et al. (2014) conducted a strong- and weak-lensing (SAWLENS hereafter; Merten et al. 2009) analysis of 19 CLASH X-ray-selected clusters, including all clusters in our X-ray-selected subsample, by combining data from 16 band HST imaging with the widefield weak-shear data analyzed in the present study. Their work is thus complementary to our wide-field weak-lensing analysis, which combines the shear and magnification effects. Merten et al. (2014) derived a $c-M$ relation for their clusters, finding a moderately significant trend of decreasing $c_{200 c}$ with increasing halo mass, which is in good agreement with $\Lambda \mathrm{CDM}$ predictions.

The square in Figure 9 represents the average concentration predicted for our X-ray-selected subsample using their $M_{200 \mathrm{c}}$ masses and best-fit $c(M, z)$ relation, demonstrating consistency between the results obtained with different lensing methods.

\subsubsection{Comparison with Previous Stacked-lensing Studies}

Our findings are in agreement with the results obtained by Okabe et al. (2013), who carried out a stacked weak-lensing analysis of $50 \mathrm{X}$-ray clusters $\left(0.15<z_{1}<0.3\right)$ from $R=0.1$ to $2.8 \mathrm{Mpc} h^{-1}$, using two-band imaging from Subaru/SuprimeCam observations. For their full sample, Okabe et al. (2013) found a mean concentration of $c_{200 c}=4.22_{-0.36}^{+0.40}$ at their effective halo mass of $M_{200 c}=(8.5 \pm 0.6) \times 10^{14} M_{\odot} h_{70}^{-1}$, which exceeds the predicted concentration from numerical simulations (Duffy et al. 2008; Zhao et al. 2009; Bhattacharya et al. 2013; De Boni et al. 2013). 
More recently, Covone et al. (2014) used the CFHT Lensing Survey (CFHTLenS; Heymans et al. 2012) shear catalog to measure the stacked shear signal of 1176 optically selected clusters $\left(0.1<z_{1}<0.6\right)$ from $R=0.1$ to $20 \mathrm{Mpc} h^{-1}$, in six bins of optical richness corresponding to the mass range $0.7 \lesssim M_{200 \mathrm{c}} /\left(10^{14} M_{\odot} h_{70}^{-1}\right) \lesssim 5$ at a median redshift of $\bar{z}_{1}=0.36$. The normalization of their $c(M)$ relation is higher than but marginally consistent with the prediction by Duffy et al. (2008) and is in closer agreement with recent simulations by Bhattacharya et al. (2013), which is qualitatively consistent with our results.

Umetsu et al. (2011b) obtained an averaged total mass profile $\langle\langle\boldsymbol{\Sigma}\rangle\rangle$ of four similar-mass, strong-lensing clusters at $\left\langle\left\langle z_{1}\right\rangle\right\rangle \simeq 0.32$ using combined strong-lensing, weak-lensing shear and magnification measurements from high-quality $H S T$ and Subaru observations. These clusters display prominent strong-lensing features, characterized by large Einstein radii of $\theta_{\text {Ein }} \gtrsim 30^{\prime \prime}$ for a fiducial source redshift of $z_{\mathrm{s}}=2$. Umetsu et al. (2011b) found that their stacked total mass profile is well described by a single NFW profile over a wide radial range, $R=40-2800 \mathrm{kpc} h^{-1}$. The mean concentration is $c_{\mathrm{vir}}=$ $7.68_{-0.40}^{+0.42}$ at $M_{\mathrm{vir}}=2.20_{-0.14}^{+0.16} \times 10^{15} M_{\odot} h_{70}^{-1}$, corresponding to an Einstein radius of $\theta_{\text {Ein }} \simeq 36^{\prime \prime}\left(z_{\mathrm{s}}=2\right)$, which is compared to our CLASH X-ray-selected subsample with $c_{\text {vir }}=5.0 \pm 0.4$, $M_{\mathrm{vir}}=(1.58 \pm 0.12) \times 10^{15} M_{\odot} h_{70}^{-1}$, and $\theta_{\text {Ein }}=15^{\prime \prime} \pm 4^{\prime \prime}$ $\left(z_{\mathrm{s}}=2\right)$. Intriguingly, the results from these two stacking studies are in good agreement with the $\Lambda$ CDM prediction for the $c_{\text {vir }}-\theta_{\text {Ein }}$ relation based on semi-analytic calculations of Oguri et al. (2012, see their Figure 10), in which clusters with larger Einstein radii are statistically more concentrated in projection.

\subsubsection{Impact of the Inclusion of Less Relaxed Clusters}

Here we examine the robustness of our results against the inclusion of less relaxed clusters. To do this, we perform a stacked-shear-only analysis by excluding those clusters likely with a lesser degree of dynamical relaxation, as indicated by their relatively higher degree of substructure (Postman et al. 2012; Lemze et al. 2013), namely, A209, A2261, RX J2248.7-4431, MACS J0329.7-0211, RX J1347.5-1145, and MACS J0744.9+3927. For the remaining subset of $10 \mathrm{X}$-ray regular clusters, the best-fit NFW parameters are obtained as $c_{200 \mathrm{c}}=3.9 \pm 0.4$ and $M_{200 \mathrm{c}}=(1.30 \pm 0.12) \times 10^{15} M_{\odot} h_{70}^{-1}$, at $\left\langle\left\langle z_{1}\right\rangle\right\rangle=0.334$. We thus find an only negligible change in the best-fit NFW parameters compared to the total uncertainties.

We note that this subset exhibits a smaller level of clusterto-cluster variations in the tangential distortion signal, and the total uncertainties in the stacked-lensing signal are dominated by statistical noise. Accordingly, we find that the uncertainties in the derived parameters here are comparable to those found for our total X-ray-selected subsample of 16 clusters.

\subsubsection{Baryonic Effects}

Our CLASH X-ray selection $\left(T_{X}>5 \mathrm{keV}\right)$ is designed to minimize the impact of baryonic effects on the cluster mass distribution. The effects of baryonic physics can in principle impact the inner halo profile ( $r \lesssim 0.1 r_{\text {vir }}$; Duffy et al. 2010) and thus modify the gravity-only $c-M$ relation, especially for less massive halos (Duffy et al. 2010; Bhattacharya et al. 2013).

In the present stacked-shear-only analysis, we examined the possible impact of adiabatic contraction by introducing a central point mass into our modeling. From this we obtained a $1 \sigma$ upper limit on the unresolved central mass of $M_{\mathrm{p}}<$
$44 \times 10^{12} M_{\odot} h_{70}^{-1}$ within $R_{\min }=200 \mathrm{kpc} h^{-1}(\mathrm{NFW}+\mathrm{pm}$ in Table 7). We find that this does not significantly impact the best-fit $M_{200 c}$ and $c_{200 c}$ values for our data, but allows for somewhat lower concentrations, $c_{200 \mathrm{c}} \gtrsim 3.2$ at $1 \sigma$. Our findings are consistent with the conclusions of Okabe et al. (2013), who obtained a tighter limit of $M_{\mathrm{p}}<17 \times 10^{12} M_{\odot} h_{70}^{-1}$ within $R_{\text {min }}=80 \mathrm{kpc} h^{-1}$ from their stacked weak-shear analysis of $50 \mathrm{X}$-ray-luminous clusters at $0.15<z<0.3$.

\subsection{Ensemble-averaged Cluster Mass Profile Shape}

\subsubsection{Halo Mass Profile (1h term)}

Since the tangential shear is a measure of the radially modulated surface mass density, which is insensitive to sheetlike structures, the stacked-shear-only analysis can provide powerful constraints on the halo structure. We find that the shape of the stacked shear profile exhibits a steepening radial trend across the radial range $200-3500 \mathrm{kpc} h^{-1}$, which is well described by the NFW, BMO (truncated-NFW), and Einasto models (Section 6.1.2), whereas the two-halo contribution to $\Delta \Sigma_{+}$is negligible across the radial range of our observations.

The Einasto shape parameter is constrained as $\alpha_{\mathrm{E}}=$ $0.191_{-0.068}^{+0.071}$, which agrees fairly well with numerical simulations: $\alpha_{\mathrm{E}}=0.175 \pm 0.046$ (Gao et al. 2012; the bestfit for the averaged profile of their nine cluster-sized halos), $\alpha_{\mathrm{E}}=0.172 \pm 0.032$ (Navarro et al. 2004, the average and dispersion of their 19 dwarf- to cluster-sized halos), $\alpha_{\mathrm{E}} \sim 0.17$ (Merritt et al. 2006; the median of their six cluster-sized halos), $\alpha_{\mathrm{E}}=0.21 \pm 0.07\left(\mathrm{M} 14 ; \alpha_{\mathrm{E}}=0.24 \pm 0.09\right.$ when fitted to the projected total mass density profiles). The fitting formula given by Gao et al. (2008) yields $\alpha_{\mathrm{E}} \sim 0.29$ for our high-mass clusters at $\left\langle\left\langle z_{1}\right\rangle\right\rangle \simeq 0.35$, which is consistent with our results within $1.3 \sigma$. Our results are also consistent with the recent stacked weak-shear analysis of $50 \mathrm{X}$-ray-luminous clusters by Okabe et al. (2013), who obtained $\alpha_{\mathrm{E}}=0.188_{-0.058}^{+0.062}$ for their sample with $M_{200 \mathrm{c}}=(8.5 \pm 0.6) \times 10^{14} M_{\odot} h_{70}^{-1}$.

Misidentification of cluster centers is a potential source of systematic errors for stacked weak-lensing measurements on small scales. George et al. (2012) examined the impact of the choice for the cluster center on the stacked weak-shear signal based on $129 \mathrm{X}$-ray-selected galaxy groups at $0<z<1$ detected in the COSMOS field. They show that the brightest or most massive galaxies near the X-ray centroids appear to best trace the center of mass of halos. Zitrin et al. (2012) analyzed the strong-lensing signature of 10,000 clusters from the Gaussian Mixture Brightest Cluster Galaxy (Hao et al. 2010) catalog, finding a small mean offset of $\simeq 13 \mathrm{kpc} h^{-1}$ between the BCG and the smoothed optical light that is assumed to trace the DM in their analysis.

Johnston et al. (2007) demonstrated that the smoothing effects of miscentering on $\Delta \Sigma_{+}$are much larger than on $\Sigma$ and produce a noticeable effect on $\Delta \Sigma_{+}$out to 10 times the typical positional offset from the cluster mass centroid (Johnston et al. 2007). This is not surprising because $\Delta \Sigma_{+}$is insensitive to flat sheetlike structures. Here our CLASH X-ray-selection criteria ensure well-defined cluster centers, reducing the smoothing effects of cluster miscentering. Assuming that the BCG-X-ray positional offset is a good proxy for the offset from the mass centroid, the smoothing effects on $\Delta \Sigma_{+}$vanish at $R \gtrsim 10 \sigma_{\text {off }} \sim 110 \mathrm{kpc} h^{-1}$ (Section 5.1), which is sufficiently smaller than the innermost measurement radius, $R_{\min }=200 \mathrm{kpc} h^{-1}$, for our stacked shear analysis. 


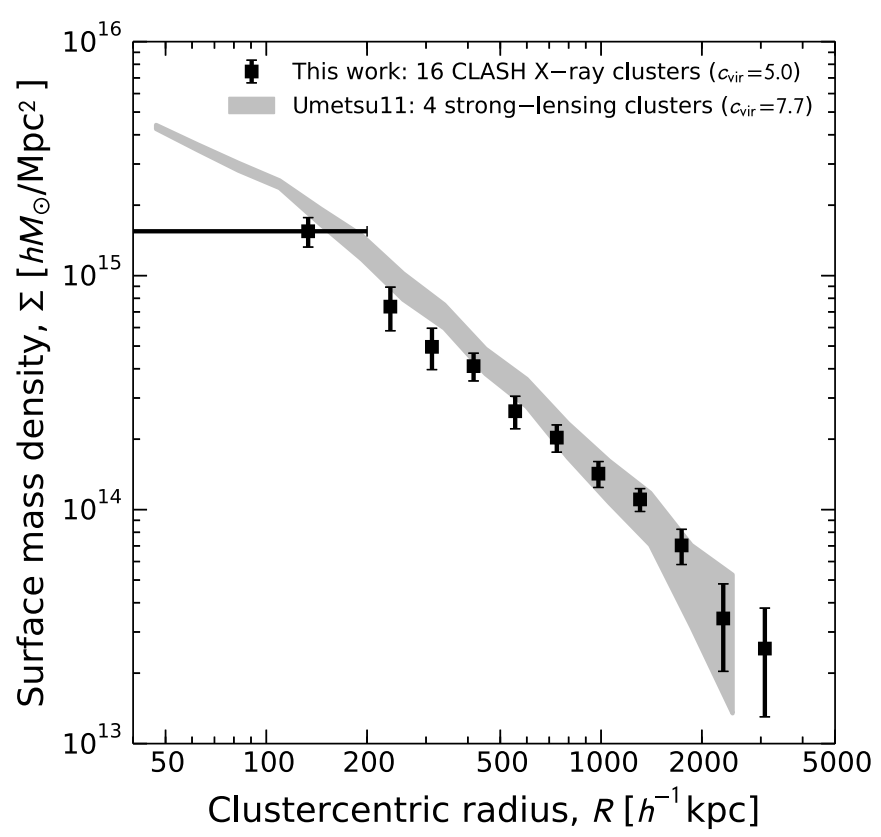

Figure 10. Comparison of stacked projected total mass profiles between different cluster samples. The squares with error bars show the results $(68 \%$ CL; Figure 7) from our stacking analysis of 16 CLASH X-ray-selected clusters at $\left\langle\left\langle z_{1}\right\rangle\right\rangle \simeq 0.35$ based on the weak-lensing shear+magnification measurements. The gray-shaded area represents the averaged total mass profile (68\% CL) of four similar-mass, strong-lensing-selected clusters at $\left\langle\left\langle z_{1}\right\rangle\right\rangle \simeq 0.32$ obtained from a combined strong-lensing, weak-lensing shear and magnification analysis of Umetsu et al. (2011b). The mean concentration of the strong-lensing sample is $c_{\mathrm{vir}}=7.7 \pm 0.4$, compared to $c_{\mathrm{vir}}=5.0 \pm 0.4$ for the CLASH X-rayselected subsample.

\subsubsection{Total Mass Profile $(1 \mathrm{~h}+2 \mathrm{~h}$ term $)$}

We compare in Figure 10 the ensemble-averaged projected mass density profile $\langle\langle\Sigma\rangle\rangle$ of our X-ray-selected subsample with the results of Umetsu et al. (2011b), who analyzed combined strong-lensing, weak-lensing shear and magnification measurements of four strong-lensing-selected clusters (Section 7.2.3), characterized by $c_{\mathrm{vir}}=7.68_{-0.40}^{+0.42}$ and $M_{\mathrm{vir}}=$ $2.20_{-0.14}^{+0.16} \times 10^{15} M_{\odot} h_{70}^{-1}$ at $\left\langle\left\langle z_{1}\right\rangle\right\rangle \simeq 0.32$. This is translated into the halo bias factor, $b_{\mathrm{h}} \simeq 10.9$, which is only $\sim 20 \%$ higher than that estimated for our X-ray-selected subsample, $b_{\mathrm{h}} \simeq 9.0$ (Section 6.2). In fact, the two cluster samples have similar "peak heights" in the linear (primordial) density field (Tinker et al. 2010): $\simeq 3.8 \sigma$ for our X-ray-selected subsample and $\simeq 4.1 \sigma$ for the Umetsu et al. (2011b) sample.

Figure 10 shows that the two samples have very similar outer mass profiles at $R \gtrsim 1 \mathrm{Mpc} h^{-1} \sim 0.5 r_{\text {vir }}$, which are sensitive to the underlying mass accretion rate or halo peak height (Diemer $\&$ Kravtsov 2014). At $R \lesssim 400 \mathrm{kpc} h^{-1}\left(\sim r_{\mathrm{s}}\right)$, on the other hand, we start to see systematic deviations between the two profiles, reflecting different degrees of projected mass concentration.

As shown by high-resolution cluster simulations of Gao et al. (2012), the asphericity of clusters can lead to large variations of up to a factor of three in the projected density $\Sigma(R)$ at a given radius $R$, depending on projection, especially within $R \sim 500 \mathrm{kpc} h^{-1}$ (see their Figure 9). Such projection effects due to cluster asphericity could explain the high apparent concentration and high central surface mass density of these four strong-lensing clusters (see also Oguri et al. 2012).

\subsection{Systematic Errors}

As described in Sections 3 and 5.4, we have taken into account several major sources of uncertainties in our error analysis. In this subsection we address other potential sources of systematic errors and discuss their possible effects on our results. In summary, we conclude that they are subdominant to the other sources we have already addressed.

\subsubsection{Number Count Slopes}

In the presence of magnification, one probes the number counts at an effectively fainter limiting magnitude of $m_{\mathrm{lim}}+$ $2.5 \log _{10} \mu$. The level of magnification is on average small in the weak regime but for our innermost bin $\theta=\left[0.9,1^{\prime} .2\right]$ reaches a median factor of $\mu \sim 1.7$, corresponding to a magnitude shift of $\delta m \sim 0.6$. Hence, we have implicitly assumed that the power-law behavior in Equation (8) persists down to $\sim 0.6 \mathrm{mag}$ fainter than $m_{\text {lim }}$, where the count slope may be shallower. For a given level of count depletion, an overestimation of the count slope could lead to an overestimation of the magnification, thus biasing the resulting mass profile. However, the count slope $s_{\text {eff }}$ for our data flattens slowly with depth varying from $s_{\text {eff }} \sim 0.15$ to $s_{\text {eff }} \sim 0.1$ from a typical magnitude limit of $m_{\lim }=25.4$ to $m_{\text {lim }}+\delta m$ (see also Umetsu et al. 2011a), so that this introduces a small correction of only $7 \%$ for the innermost bin, much smaller compared to our noisy depletion measurements with a $\sim 54 \%$ median uncertainty, corresponding to $54 \% / \sqrt{N_{\mathrm{cl}}} \sim 14 \%$ when all clusters are combined. Therefore, we conclude that the effect of this correction is subdominant with respect to the total uncertainty.

\subsubsection{Background-level Determination}

The background density parameter $\bar{n}_{\mu}$ for the count-depletion analysis has been estimated from the red galaxy counts in the outer region $\theta=\left[10^{\prime}, \theta_{\max }\right]$ (Table 5). We find from the stacked mass profile that the mean convergence at $\theta \geqslant 10^{\prime}$, where we have estimated $\bar{n}_{\mu}$, is $\kappa=(8 \pm 4) \times 10^{-3}$ at $\left\langle\left\langle z_{1}\right\rangle\right\rangle \simeq 0.35$. This corresponds to a depletion signal of $\delta n_{\mu} / \bar{n}_{\mu} \approx\left(5\left\langle s_{\text {eff }}\right\rangle-2\right) \kappa \sim$ -0.01 with the mean count slope $\left\langle s_{\text {eff }}\right\rangle \sim 0.15$ of our sample, indicating that the estimated $\bar{n}_{\mu}$ is biased low by $1 \%$. This level of the signal offset, however, is smaller than the calibration uncertainties of $\sigma\left(\bar{n}_{\mu}\right) / \bar{n}_{\mu}=(2-8) \%$ for an individual cluster. Hence, for all clusters in our sample, the offset signal lies within the prior range considered, $\left[\bar{n}_{\mu}-\sigma\left(\bar{n}_{\mu}\right), \bar{n}_{\mu}+\sigma\left(\bar{n}_{\mu}\right)\right]$ (Section 3.3.1). In fact, we find that the ML (best-fit) estimates of $\bar{n}_{\mu}$, as constrained by the combined shear+magnification data, are on average $(1.0 \pm 0.6) \%$ larger than the values estimated from the counts at $\left[10^{\prime}, \theta_{\max }\right]$, so that the lensing signal is consistently recovered.

This analysis demonstrates that the background level determination is not critically sensitive to our calibration prior on the background density parameter $\bar{n}_{\mu}$, but driven by the combined shear+magnification data. ${ }^{36}$

\section{SUMMARY AND CONCLUSIONS}

We have presented a joint shear-and-magnification weaklensing analysis of a sample of $16 \mathrm{X}$-ray-regular and 4 highmagnification galaxy clusters at $0.19 \lesssim z \lesssim 0.69$ targeted in the CLASH survey (Postman et al. 2012). Our analysis uses

\footnotetext{
36 For example, the observable distortion in the nonlinear regime is not invariant with adding a mass-sheet component, so that the lensing constraints in the nonlinear regime can help break the parameter degeneracy.
} 
deep wide-field multi-color imaging obtained primarily with Subaru/Suprime-Cam.

From a stacked-shear-only analysis of the X-ray-selected subsample, we have detected the ensemble-averaged lensing signal $\left\langle\left\langle\Delta \boldsymbol{\Sigma}_{+}\right\rangle\right\rangle$with a total $\mathrm{S} / \mathrm{N}$ of $\simeq 25$ in the radial range of 200-3500 kpc $h^{-1}$ (Figure 5), providing integrated constraints on the halo profile shape and $c-M$ relation. The shape of the stacked $\left\langle\left\langle\Delta \boldsymbol{\Sigma}_{+}\right\rangle\right\rangle$profile exhibits a steepening radial trend across the radial range, which is well described by a family of standard density profiles predicted for DM-dominated halos in gravitational equilibrium (Table 7). The best-fit Einasto shape parameter is $\alpha_{\mathrm{E}}=0.191_{-0.068}^{+0.071}$, which is consistent with the NFW-equivalent Einasto parameter of $\sim 0.18$.

For the NFW model, we constrain the mean concentration of our X-ray-selected subsample to lie in the range $c_{200 \mathrm{c}}=$ $4.01_{-0.32}^{+0.35}$ at $M_{200 c}=1.34_{-0.09}^{+0.10} \times 10^{15} M_{\odot} h_{70}^{-1}$ (Table 7), corresponding to the Einstein radius of $\theta_{\text {Ein }}=15^{\prime \prime} \pm 4^{\prime \prime}\left(z_{\mathrm{s}}=2\right)$. Accounting for the CLASH selection function based on X-ray morphology and projection effects inherent in lensing observations (M14), we find an excellent agreement between observations and theoretical predictions (Table 8). Our stacked constraints on the $c-M$ relation are slightly higher than but in agreement with the results from the SAWLENS analysis of 19 CLASH X-ray-selected clusters (Merten et al. 2014), demonstrating consistency between the results obtained with different lensing methods (Section 7.2.2).

We have reconstructed model-free projected mass profiles $\boldsymbol{\Sigma}$ of all CLASH clusters (Figure 3 ) from a joint likelihood analysis of consistent shear-and-magnification measurements (Figure 2). The cluster masses were estimated at several characteristic radii by fitting the observed $\boldsymbol{\Sigma}$ profiles with a spherical NFW density profile (see Table 6). The results are subject to a systematic uncertainty of $\pm 8 \%$ in the overall mass calibration (Section 5.4.4).

We have also derived an ensemble-averaged total projected mass profile $\langle\langle\Sigma\rangle\rangle$ of our X-ray-selected subsample by stacking their individual mass profiles $\boldsymbol{\Sigma}$ (Figure 7 ). The averaged total mass profile is shown to be consistent with our shear-based halomodel predictions for the total matter distribution $\Sigma_{1 \mathrm{~h}}+\Sigma_{2 \mathrm{~h}}$, including the effects of surrounding LSS as a two-halo term $\Sigma_{2 \mathrm{~h}}$, thus establishing further consistency in the context of the standard $\Lambda$ CDM model.

An accurate determination of the cluster density profile over the full radial range, from a combination of strong- and weaklensing information, is crucial for testing DM and alternative gravity paradigms. The CLASH survey (Postman et al. 2012) is designed to generate such multi-scale, multi-probe lensing data using high-resolution 16 band HST imaging and widefield ground-based observations for a sizable sample of clusters (Merten et al. 2014). A stacked cluster analysis, combining all lensing-related effects in the cluster regime (Umetsu 2013), will be a crucial next step toward a definitive determination of the ensemble-averaged cluster mass profile from the inner core to beyond the virial radius, providing a firm basis for a detailed comparison with the $\Lambda \mathrm{CDM}$ paradigm and a wider examination of alternative scenarios (Woo \& Chiueh 2009; Narikawa et al. 2013; Beraldo e Silva et al. 2013).

We thank the anonymous referee for the careful reading of the manuscript and constructive suggestions. We acknowledge fruitful discussions with Nobuhiro Okabe, Masamune Oguri, Mauro Sereno, Jean Coupon, and Hitoshi Hanami. We thank Ole Host for providing very helpful comments on the manuscript. We are grateful to all members of the CLASH team who enabled us to carry out the work. We acknowledge the Subaru Support Astronomers, plus Justice Bruursema, Kai-Yang Lin, and Hiroaki Nishioka, for assistance with our Subaru observations. We thank Nick Kaiser and Masamune Oguri for making their IMCAT and GLAFIC packages publicly available. This work is partially supported by the Academia Sinica Career Development Award and by the Ministry of Science and Technology of Taiwan through grants NSC100-2112M-001-008-MY3 and MOST 103-2112-M-001-030-MY3. J.M. acknowledges support from the Jet Propulsion Laboratory, California Institute of Technology, under a contract with the National Aeronautics and Space Administration. D.G. and S.S. were supported by SFB-Transregio 33 "The Dark Universe" by the Deutsche Forschungsgemeinschaft (DFG) and the DFG cluster of excellence "Origin and Structure of the Universe." Support for A.Z. was provided by NASA through Hubble Fellowship grant \#HST-HF-51334.01-A awarded by STScI. The Dark Cosmology Centre is funded by the Danish National Research Foundation.

\section{REFERENCES}

Abell, G. O. 1958, ApJS, 3, 211

Abell, G. O., Corwin, H. G., Jr., \& Olowin, R. P. 1989, ApJS, 70, 1

Adelman-McCarthy, J. K., Agüeros, M. A., Allam, S. S., et al. 2008, ApJS, 175,297

Applegate, D. E., von der Linden, A., Kelly, P. L., et al. 2014, MNRAS, 439, 48 Baltz, E. A., Marshall, P., \& Oguri, M. 2009, JCAP, 1, 15

Bartelmann, M., \& Schneider, P. 2001, PhR, 340, 291

Becker, M. R., \& Kravtsov, A. V. 2011, ApJ, 740, 25

Beers, T. C., Flynn, K., \& Gebhardt, K. 1990, AJ, 100, 32

Benítez, N. 2000, ApJ, 536, 571

Benítez, N., Ford, H., Bouwens, R., et al. 2004, ApJS, 150, 1

Beraldo e Silva, L. J., Lima, M., \& Sodré, L. 2013, MNRAS, 436, 2616

Bertin, E. 2006, in ASP Conf. Ser. 351, Astronomical Data Analysis Software and Systems XV, ed. C. Gabriel, C. Arviset, D. Ponz, \& S. Enrique (San Francisco, CA: ASP), 112

Bertin, E., \& Arnouts, S. 1996, A\&AS, 117, 393

Bertin, E., Mellier, Y., Radovich, M., et al. 2002, in ASP Conf. Ser. 281, Astronomical Data Analysis Software and Systems XI, ed. D. A. Bohlender, D. Durand, \& T. H. Handley (San Francisco, CA: ASP), 228

Bhattacharya, S., Habib, S., Heitmann, K., \& Vikhlinin, A. 2013, ApJ, 766, 32 Bigelow, B. C., \& Dressler, A. M. 2003, Proc. SPIE, 4841, 1727

Biviano, A., Rosati, P., Balestra, I., et al. 2013, A\&A, 558, A1

Blanchard, P. K., Bayliss, M. B., McDonald, M., et al. 2013, ApJ, 772, 24

Blumenthal, G. R., Faber, S. M., Flores, R., \& Primack, J. R. 1986, ApJ, 301, 27

Broadhurst, T., Takada, M., Umetsu, K., et al. 2005, ApJL, 619, L143

Broadhurst, T., Umetsu, K., Medezinski, E., Oguri, M., \& Rephaeli, Y. 2008, ApJL, 685, L9

Broadhurst, T. J., Taylor, A. N., \& Peacock, J. A. 1995, ApJ, 438, 49

Bullock, J. S., Kolatt, T. S., Sigad, Y., et al. 2001, MNRAS, 321, 559

Cavaliere, A., \& Fusco-Femiano, R. 1978, A\&A, 70, 677

Coe, D., Benítez, N., Sánchez, S. F., et al. 2006, AJ, 132, 926

Coe, D., Umetsu, K., Zitrin, A., et al. 2012, ApJ, 757, 22

Coupon, J., Broadhurst, T., \& Umetsu, K. 2013, ApJ, 772, 65

Covone, G., Sereno, M., Kilbinger, M., \& Cardone, V. F. 2014, ApJL, 784, L25 De Boni, C., Ettori, S., Dolag, K., \& Moscardini, L. 2013, MNRAS, 428, 2921

Diemer, B., \& Kravtsov, A. V. 2014, ApJ, 789, 1

Donahue, M., Voit, G. M., Mahdavi, A., et al. 2014, ApJ, 794, 136

Duffy, A. R., Schaye, J., Kay, S. T., \& Dalla Vecchia, C. 2008, MNRAS, 390, L64

Duffy, A. R., Schaye, J., Kay, S. T., et al. 2010, MNRAS, 405, 2161

Ebeling, H., Barrett, E., Donovan, D., et al. 2007, ApJL, 661, L33

Ebeling, H., Edge, A. C., \& Henry, J. P. 2001, ApJ, 553, 668

Ebeling, H., Edge, A. C., Mantz, A., et al. 2010, MNRAS, 407, 83

Einasto, J. 1965, TrAlm, 5, 87

Ford, H. C., Clampin, M., Hartig, G. F., et al. 2003, Proc. SPIE, 4854, 81

Ford, J., Hildebrandt, H., Van Waerbeke, L., et al. 2012, ApJ, 754, 143

Gao, L., Navarro, J. F., Cole, S., et al. 2008, MNRAS, 387, 536

Gao, L., Navarro, J. F., Frenk, C. S., et al. 2012, MNRAS, 425, 2169 
George, M. R., Leauthaud, A., Bundy, K., et al. 2012, ApJ, 757, 2

Gnedin, O. Y., Kravtsov, A. V., Klypin, A. A., \& Nagai, D. 2004, ApJ, 616, 16 Graham, A. W., Merritt, D., Moore, B., Diemand, J., \& Terzić, B. 2006, AJ, 132,2701

Gruen, D., Brimioulle, F., Seitz, S., et al. 2013, MNRAS, 432, 1455

Gunn, J. E., \& Gott, J. R. I. 1972, ApJ, 176, 1

Haiman, Z., Mohr, J. J., \& Holder, G. P. 2001, ApJ, 553, 545

Hanami, H., Ishigaki, T., Fujishiro, N., et al. 2012, PASJ, 64, 70

Hao, J., McKay, T. A., Koester, B. P., et al. 2010, ApJS, 191, 254

Hattori, M., Kneib, J., \& Makino, N. 1999, PThPS, 133, 1

Heymans, C., Van Waerbeke, L., Bacon, D., et al. 2006, MNRAS, 368, 1323

Heymans, C., Van Waerbeke, L., Miller, L., et al. 2012, MNRAS, 427, 146

Hildebrandt, H., Muzzin, A., Erben, T., et al. 2011, ApJL, 733, L30

Hjorth, J., \& Williams, L. L. R. 2010, ApJ, 722, 851

Hoekstra, H. 2003, MNRAS, 339, 1155

Hoekstra, H., Bartelmann, M., Dahle, H., et al. 2013, SSRv, 177, 75

Hoekstra, H., Hartlap, J., Hilbert, S., \& van Uitert, E. 2011, MNRAS, 412, 2095

Ilbert, O., Capak, P., Salvato, M., et al. 2009, ApJ, 690, 1236

Ilbert, O., Salvato, M., Le Floc'h, E., et al. 2010, ApJ, 709, 644

Jing, Y. P., \& Suto, Y. 2000, ApJL, 529, L69

Johnston, D. E., Sheldon, E. S., Wechsler, R. H., et al. 2007, arXiv:0709.1159

Jouvel, S., Host, O., Lahav, O., et al. 2014, A\&A, 562, A86

Kaiser, N. 1995, ApJL, 439, L1

Kaiser, N., Squires, G., \& Broadhurst, T. 1995, ApJ, 449, 460

Kelly, P. L., von der Linden, A., Applegate, D. E., et al. 2014, MNRAS, 439, 28

Kimble, R. A., MacKenty, J. W., O'Connell, R. W., \& Townsend, J. A 2008, Proc. SPIE, 7010, 70101E

Kitayama, T., \& Suto, Y. 1996, ApJ, 469, 480

Komatsu, E., Smith, K. M., Dunkley, J., et al. 2011, ApJS, 192, 18

Lapi, A., \& Cavaliere, A. 2009, ApJ, 692, 174

Lau, E. T., Nagai, D., Kravtsov, A. V., \& Zentner, A. R. 2011, ApJ, 734, 93

Lemze, D., Postman, M., Genel, S., et al. 2013, ApJ, 776, 91

Lilly, S. J., Le Fèvre, O., Renzini, A., et al. 2007, ApJS, 172, 70

Ludlow, A. D., Navarro, J. F., Boylan-Kolchin, M., et al. 2013, MNRAS, 432, 1103

Massey, R., Heymans, C., Bergé, J., et al. 2007, MNRAS, 376, 13

Mead, J. M. G., King, L. J., Sijacki, D., et al. 2010, MNRAS, 406, 434

Medezinski, E., Broadhurst, T., Umetsu, K., Benítez, N., \& Taylor, A. 2011, MNRAS, 414, 1840

Medezinski, E., Broadhurst, T., Umetsu, K., et al. 2010, MNRAS, 405, 257

Medezinski, E., Umetsu, K., Nonino, M., et al. 2013, ApJ, 777, 43

Meneghetti, M., Fedeli, C., Pace, F., Gottlöber, S., \& Yepes, G. 2010a, A\&A, 519, A90

Meneghetti, M., Rasia, E., Merten, J., et al. 2010b, A\&A, 514, A93

Meneghetti, M., Rasia, E., Vega, J., et al. 2014, ApJ, in press, arXiv:1404.1384

Merritt, D., Graham, A. W., Moore, B., Diemand, J., \& Terzić, B. 2006, AJ, 132,2685

Merten, J., Cacciato, M., Meneghetti, M., Mignone, C., \& Bartelmann, M. 2009, A\&A, 500, 681

Merten, J., Meneghetti, M., Postman, M., et al. 2014, arXiv:1404.1376

Miyazaki, S., Komiyama, Y., Sekiguchi, M., et al. 2002, PASJ, 54, 833

Narikawa, T., Kobayashi, T., Yamauchi, D., \& Saito, R. 2013, PhRvD, 87,124006

Narikawa, T., \& Yamamoto, K. 2012, JCAP, 5, 16

Navarro, J. F., Frenk, C. S., \& White, S. D. M. 1996, ApJ, 462, 563
Navarro, J. F., Hayashi, E., Power, C., et al. 2004, MNRAS, 349, 1039

Navarro, J. F., Ludlow, A., Springel, V., et al. 2010, MNRAS, 402, 21

Neto, A. F., Gao, L., Bett, P., et al. 2007, MNRAS, 381, 1450

Newman, A. B., Treu, T., Ellis, R. S., et al. 2013, ApJ, 765, 24

Nonino, M., Dickinson, M., Rosati, P., et al. 2009, ApJS, 183, 244

Oguri, M. 2010, PASJ, 62, 1017

Oguri, M., Bayliss, M. B., Dahle, H., et al. 2012, MNRAS, 420, 3213

Oguri, M., \& Hamana, T. 2011, MNRAS, 414, 1851

Oguri, M., Hennawi, J. F., Gladders, M. D., et al. 2009, ApJ, 699, 1038

Oguri, M., \& Takada, M. 2011, PhRvD, 83, 023008

Oguri, M., Takada, M., Umetsu, K., \& Broadhurst, T. 2005, ApJ, 632, 841

Okabe, N., Smith, G. P., Umetsu, K., Takada, M., \& Futamase, T. 2013, ApJL, 769, L35

Okabe, N., Takada, M., Umetsu, K., Futamase, T., \& Smith, G. P. 2010, PASJ, 62,811

Okabe, N., \& Umetsu, K. 2008, PASJ, 60, 345

Okura, Y., \& Futamase, T. 2012, ApJ, 748, 112

Postman, M., Coe, D., Benítez, N., et al. 2012, ApJS, 199, 25

Rasia, E., Meneghetti, M., Martino, R., et al. 2012, NJPh, 14, 055018

Rozo, E., Bartlett, J. G., Evrard, A. E., \& Rykoff, E. S. 2014, MNRAS, 438, 78

Rozo, E., \& Schmidt, F. 2010, arXiv:1009.5735

Schlegel, D. J., Finkbeiner, D. P., \& Davis, M. 1998, ApJ, 500, 525

Schneider, P., King, L., \& Erben, T. 2000, A\&A, 353, 41

Schneider, P., \& Seitz, C. 1995, A\&A, 294, 411

Seitz, C., \& Schneider, P. 1997, A\&A, 318, 687

Sereno, M., \& Covone, G. 2013, MNRAS, 434, 878

Sereno, M., \& Umetsu, K. 2011, MNRAS, 416, 3187

Shaw, L. D., Weller, J., Ostriker, J. P., \& Bode, P. 2006, ApJ, 646, 815

Smith, R. E., Peacock, J. A., Jenkins, A., et al. 2003, MNRAS, 341, 1311

Taylor, A. N., Dye, S., Broadhurst, T. J., Benitez, N., \& van Kampen, E. 1998, ApJ, 501, 539

Taylor, J. E., \& Navarro, J. F. 2001, ApJ, 563, 483

Tinker, J. L., Robertson, B. E., Kravtsov, A. V., et al. 2010, ApJ, 724, 878

Umetsu, K. 2013, ApJ, 769, 13

Umetsu, K., Birkinshaw, M., Liu, G.-C., et al. 2009, ApJ, 694, 1643

Umetsu, K., \& Broadhurst, T. 2008, ApJ, 684, 177

Umetsu, K., Broadhurst, T., Zitrin, A., Medezinski, E., \& Hsu, L. 2011a, ApJ, 729,127

Umetsu, K., Broadhurst, T., Zitrin, A., et al. 2011b, ApJ, 738, 41

Umetsu, K., \& Futamase, T. 2000, ApJL, 539, L5

Umetsu, K., Medezinski, E., Broadhurst, T., et al. 2010, ApJ, 714, 1470

Umetsu, K., Medezinski, E., Nonino, M., et al. 2012, ApJ, 755, 56

Umetsu, K., Tada, M., \& Futamase, T. 1999, PThPS, 133, 53

van Waerbeke, L. 2000, MNRAS, 313, 524

Van Waerbeke, L., Hildebrandt, H., Ford, J., \& Milkeraitis, M. 2010, ApJL, 723, L13

von der Linden, A., Allen, M. T., Applegate, D. E., et al. 2014, MNRAS, 439, 2 Woo, T., \& Chiueh, T. 2009, ApJ, 697, 850

Wright, C. O., \& Brainerd, T. G. 2000, ApJ, 534, 34

Zemcov, M., Blain, A., Cooray, A., et al. 2013, ApJL, 769, L31

Zhao, D. H., Jing, Y. P., Mo, H. J., \& Börner, G. 2009, ApJ, 707, 354

Zitrin, A., Bartelmann, M., Umetsu, K., Oguri, M., \& Broadhurst, T. 2012, MNRAS, 426, 2944

Zitrin, A., Broadhurst, T., Coe, D., et al. 2011, ApJ, 742, 117 